\title{
Deep Exclusive Pseudoscalar Meson Production at Jefferson Lab Hall C
}

\author{
A Thesis \\ Submitted to the Faculty of Graduate Studies and Research \\ In Partial Fulfillment of the Requirements \\ For the Degree of
}

Master of Science

in

Physics

University of Regina

By

Samip Basnet

Regina, Saskatchewan

September, 2017

(C)2017 - Samip Basnet

All rights reserved. 


\section{UNIVERSITY OF REGINA \\ FACULTY OF GRADUATE STUDIES AND RESEARCH SUPERVISORY AND EXAMINING COMMITTEE}

Samip Basnet, candidate for the degree of Master of Science in Physics, has presented a thesis titled, Deep Exclusive Pseudoscalar Meson Production at Jefferson Lab Hall C, in an oral examination held on August 31, 2017. The following committee members have found the thesis acceptable in form and content, and that the candidate demonstrated satisfactory knowledge of the subject material.

External Examiner: $\quad$ Dr. Andrei Volodin, Department of Mathematics \& Statistics

Supervisor: Dr. Garth Huber, Department of Physics

Committee Member: Dr. Mauricio Barbi, Department of Physics

Committee Member: Dr. Aram Teymurazyan, Department of Physics

Committee Member: Dr. Nikolay Kolev, Department of Physics

Chair of Defense: Dr. David Gerhard, Department of Computer Science 


\begin{abstract}
Measurements of exclusive meson production are a useful tool in the study of hadronic structure. In particular, one can discern the relevant degrees of freedom at different distance scales through these studies. In the transition region between low momentum transfer (where a description of hadronic degrees of freedom in terms of effective hadronic Lagrangians is valid) and high momentum transfer (where the degrees of freedom are quarks and gluons), the predictive power of Quantum Chromodynamics (QCD), the theory of the strong interaction, is limited due to the absence of a complete solution. Thus, one has to rely upon experimental data from the non-perturbative intermediate-energy regime to thoroughly understand the onset of perturbative QCD (pQCD) as the momentum transfer is increased.

This work involves two deep exclusive meson electroproduction experiments at Jefferson Lab (JLab). The $p\left(e, e^{\prime} \pi^{+}\right) n$ reaction is studied at fixed $Q^{2}$ and $W$ of $2.5 \mathrm{GeV}^{2}$ and 2.0 $\mathrm{GeV}$, respectively, while varying the four momentum transfer to the nucleon $-t$ from 0.2 to $2.1 \mathrm{GeV}^{2}$. As $-t$ is increased, the hadronic interaction scale is reduced independently of the observation scale of the virtual photon, providing valuable information about the hardscattering process in general. The data was taken at JLab Hall C in 2003, as a part of the experiment E01-004, $\mathrm{F}_{\pi^{-}}$2, using the High Momentum Spectrometer (HMS) and Short Orbit Spectrometer (SOS), and in this work, the results of the differential cross section analysis are presented and compared to prior data, as well as two theoretical models. Using these results over a wide $-t$ range, the transition from hard to soft QCD is also studied.

In addition, the $p\left(e, e^{\prime} K^{+}\right) \Lambda\left(\Sigma^{0}\right)$ reactions are also studied. Despite their importance in elucidating the reaction mechanism underlying strangeness production, we still do not have complete understanding of these reactions above the resonance region. The experiment, E1209-011, intends to perform, for the first time, a full Rosenbluth (L/T/LT/TT) separation of $p\left(e, e^{\prime} K^{+}\right) \Lambda\left(\Sigma^{0}\right)$ cross sections above the resonance region using the newly upgraded standard equipment, Super High Momentum Spectrometer (SHMS) at JLab Hall C. The separated
\end{abstract}


data will allow us to better understand the Kaon production reaction mechanism and the hard-soft QCD transition in exclusive processes. The kinematic settings being studied in the experiment ranges from $Q^{2}$ of 0.4 to $5.5 \mathrm{GeV}^{2}, W$ of 2.3 to $3.1 \mathrm{GeV}$, and $-t$ of 0.06 to 0.53 $\mathrm{GeV}^{2}$. Here, the results from some pre-experimental studies with regards to estimations of singles rates as well as real and accidental coincidence rates are presented, using two different models. The implications of these projections on the runplan for the experiment are also discussed. 


\section{Acknowledgments}

Foremost, I would like to express the deepest gratitude to my thesis advisor, Prof. Garth M. Huber, for the continuous support throughout my Masters study and research. Without his methodical guidance and his round-the-clock availability, the completion of this project would not have been feasible. I could not have imagined having a better supervisor and a great role-model like him during my time in Regina. I would also like to extend my appreciation to my defense committee members for taking time out of their busy schedules to read through this work. I believe their expert suggestions markedly improved this work.

I have to thank the entire $\mathrm{F}_{\pi}-2$ collaboration for allowing me the opportunity to work on the high $-t$ data. In particular, Tanja Horn and Dave Gaskell for their replay effort to get the ntuples. Along with Ahmed Zafar and Pete Markowitz, Tanja and her research group were also helpful in providing valuable comments during the initial stages of analysis to estimate rates for the upcoming experiment E12-09-011.

I had the privilege to work with a fantastic group of scientists, staffs, and students at Jefferson Lab. I would like to express my appreciation to Kijun Park for stimulating discussions on high $-t$ physics as well as sharing his results from Hall B. I must thank Brad Sawatzky for advising and keeping an eye on me, while at the lab. I also want to express my gratitude to Hall C post-doc, Eric Pooser, for letting me join him on the arduous task of stringing the broken HMS drift chamber.

Special thanks are due to Wenliang Li for tolerating my myriad questions and cries for help with a smile, both on a professional and personal level. The daybreak trips to A\&W will surely be missed. Thanks also to my colleagues in Regina: Dilli Raj Poudyal, Ryan Ambrose and Rory Evans, as well as to all the faculty members, staffs, and students in the Dept. of Physics. I would like to acknowledge NSERC and FGSR for all the funding support.

Finally, I wish to thank my family for their unending love and support. The completion of this work would have been much more difficult without them. 
Dedicated to my grandfather, S. S. Basnyat. 


\section{Contents}

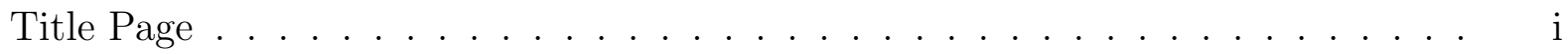

Abstract ............................... ii

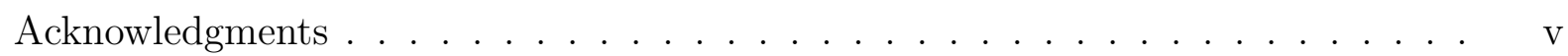

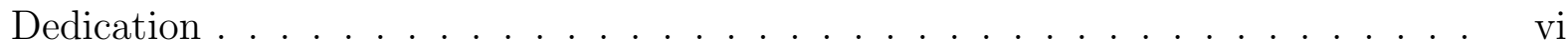

Table of Contents . . . . . . . . . . . . . . . . . vii

List of Figures . . . . . . . . . . . . . . . . . . . . . . . . . . . . . . . . .

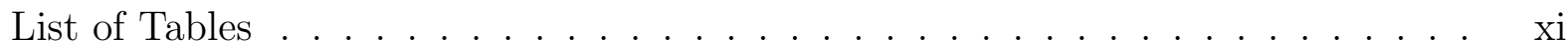

1 Introduction $\quad 1$

1.1 Deep Inelastic Exclusive Reactions . . . . . . . . . . . . . . . . . . . . . 5

1.1.1 Relevant Kinematics Quantities . . . . . . . . . . . . . . . . 9 9

1.1 .2 Cross Sections . . . . . . . . . . . . . . . . . . . 11

2 Analysis of exclusive $\pi^{+}$electroproduction data at high $-t \quad 14$

2.1 Experiment and Setup . . . . . . . . . . . . . . . . . 15

2.1.1 Experiment Kinematics . . . . . . . . . . . . . . . . . . . . . . 16

2.1 .2 Accelerator . . . . . . . . . . . . . . . . . 19

2.1.3 Cryogenic Target . . . . . . . . . . . . . . . . . . 21

2.1 .4 Spectrometers . . . . . . . . . . . . . . . . 21

2.1.5 Detector Packages . . . . . . . . . . . . . . . . . . . 25

2.1.6 Trigger System and Data Acquisition . . . . . . . . . . . . . . . 29

2.2 Data Analysis . . . . . . . . . . . . . . . . . . . . . . 30

2.2.1 Particle Identification (PID) and Event Selection . . . . . . . . . 31

2.2.2 Spectrometer Acceptance Reconstruction . . . . . . . . . . . . . . . . 33

2.2.3 Subtraction of Backgrounds . . . . . . . . . . . . . . . . 35

2.2 .4 Analysis Cuts . . . . . . . . . . . . . . . . . . 40

2.2.5 Efficiencies and Other Corrections . . . . . . . . . . . . . 41

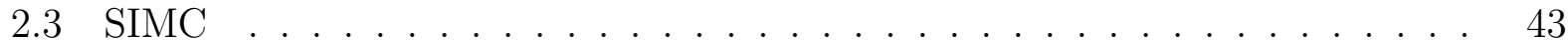

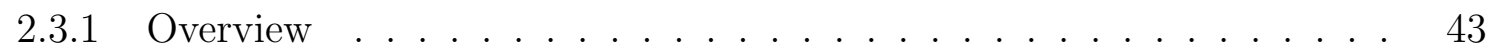

2.3.2 Data and Monte Carlo comparison . . . . . . . . . . . . . . 45

2.4 Determination of the cross section . . . . . . . . . . . . . . 50

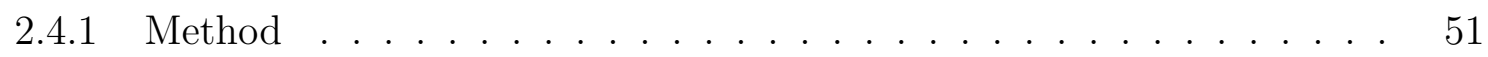

2.4.2 Model cross section . . . . . . . . . . . . . . . . . . . 53

2.4 .3 Estimate of Uncertainties . . . . . . . . . . . . . . 56 
2.5 Results . . . . . . . . . . . . . . . . . . . . 58

2.5.1 Comparison with prior data . . . . . . . . . . . . . . 59

2.5.2 Theoretical models . . . . . . . . . . . . . . . . . . . 59

3 Projections for $p\left(e, e^{\prime} K^{+}\right) \Lambda, \Sigma^{0}$ Experiment $\quad 63$

3.1 Experimental Overview . . . . . . . . . . . . . . . . . . 64

3.1 .1 Spectrometers . . . . . . . . . . . . . . . 65

3.2 Proposed Kinematics . . . . . . . . . . . . . . . . . . . . . . . . . . . . . . . . . . . . . . .

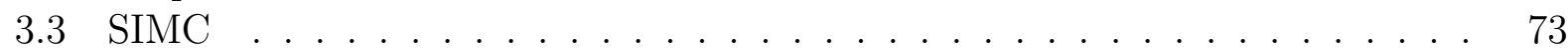

3.3.1 Use of theoretical models in the Projections . . . . . . . . . . 73

3.4 Data Analysis . . . . . . . . . . . . . . . . . . . . . 74

3.4.1 Spectrometer Acceptance . . . . . . . . . . . . . . . . . . 74

$3.4 .2 Q^{2}-W$ Coverage . . . . . . . . . . . . . . . . 76

3.4.3 Missing Mass Separation of $\Lambda$ and $\Sigma^{0} \ldots \ldots \ldots \ldots$. . . . . . . . . . . . . . . . . 77

3.4.4 Analysis Cuts . . . . . . . . . . . . . . . . . . . 81

3.5 Projected Real Coincidence Rates . . . . . . . . . . . . . . . . . . . 82

3.6 Projected Dominant Backgrounds . . . . . . . . . . . . . . . . 84

4 Discussion and Conclusion $\quad 88$

4.1 Summary for $p\left(e, e^{\prime} \pi^{+}\right) n$ analysis at high $-t \ldots \ldots \ldots$. . . . . . 89

4.2 Summary of exclusive $K^{+}$electroproduction rates projections . . . . . . . 94

$\begin{array}{ll}\text { Bibliography } & 97\end{array}$

$\begin{array}{ll}\text { A Updated beam time schedule } & 101\end{array}$ 


\section{List of Figures}

1.1 The strong coupling constant as a function of the momentum transfer $Q(\mathrm{GeV})$. 2

1.2 Exclusive $\pi^{+}$electroproduction via deep inelastic scattering . . . . . . . . . 6

1.3 Total collision cross sections as a function of invariant mass, $W$, for $\pi^{-} p$ and $K^{+} n$ collisions. . . . . . . . . . . . . . . . 7

1.4 Kinematics for $p\left(e, e^{\prime} \pi^{+}\right) n$ reaction in the lab frame . . . . . . . . . 9

2.1 Schematic representation of the Jefferson Lab site . . . . . . . . . . . . 15

2.2 Polar plot of the $|t|-\phi_{\pi}$ phase space coverage of the experiment. . . . . . . 17

2.3 Beam Line Elements . . . . . . . . . . . . . . . . . . . . . . . . . 20

2.4 Experimental Setup . . . . . . . . . . . . . . . . . . . . . . 22

2.5 Schematic side view of the HMS detector stack . . . . . . . . . . . . . . . . . . . . . . . .

2.6 Schematic of the Hall C trigger system . . . . . . . . . . . . . . . . . . . . . . . . . . . . . . . . . . .

2.7 Electron identification with the SOS. . . . . . . . . . . . . . . . . . . . . . . . . . . .

2.8 Pion identification with the HMS. . . . . . . . . . . . . . . . . 33

2.9 Spectrometer co-ordinates systems . . . . . . . . . . . . . . . . . . 35

$2.10 Q^{2}-W$ coverage at $-t=0.272$ and $2.127 \mathrm{GeV}^{2}$ settings . . . . . . . . . 36

2.11 A sample $\beta_{\text {ToF }}$ versus cointime distribution . . . . . . . . . . . . . . . . . . 37

2.12 Dummy target subtraction using ssztar distribution. . . . . . . . . . . . . . 39

2.13 Comparison of data and SIMC for HMS reconstructed quantities . . . . . . . 47

2.14 Comparison of data and SIMC for reconstructed physics quantities . . . . . . 48

2.15 Comparison of data and SIMC for the missing mass distribution . . . . . . . 50

2.16 Iteration results using the starting model input cross section . . . . . . . . . 54

2.17 The experimental cross section and yield ratios for the final iteration . . . . 55

2.18 The experimental cross section results comparison . . . . . . . . . . . . . 60

3.1 Experimental Setup . . . . . . . . . . . . . . . . . . . 64

3.2 SHMS detector stack . . . . . . . . . . . . . . . . . 66

3.3 Schematic representation of the $p\left(e, e^{\prime} K^{+}\right) \Lambda, \Sigma^{0}$ reaction in the lab frame. . . 68

$3.4 \mathrm{Q}^{2}-\mathrm{x}_{B}$ phase space available for $\mathrm{L}-\mathrm{T}$ separation in Hall $\mathrm{C}$ at $12 \mathrm{GeV}$ using the $H M S+S H M S$ combination. . . . . . . . . . . . . . . . 69

3.5 Simulated $t-\phi_{K}$ distribution for $Q^{2}=2.0 \mathrm{GeV}^{2}$ at high $\varepsilon$ setting. . . . . . . 72

3.6 Normalized distributions for the SHMS reconstructed target quantities. . . . 75

3.7 Simulated $Q^{2}-W$ phase space for high and low $\varepsilon$ settings. . . . . . . . . . . 77

3.8 Missing mass distribution for $\Lambda$ and $\Sigma^{0}$ final states. . . . . . . . . . . . . 78 
3.9 Contamination of the $\Lambda$ missing mass tail in the $\Sigma^{0}$ missing mass distribution. 79

3.10 Missing mass resolution as a function of $Q^{2}$. . . . . . . . . . . . . . . . 80

3.11 Comparison of real coincidence rates calculated using the VR model and "Old Kaon" model. . . . . . . . . . . . . . . . . . . . . . . . . . 83

4.1 Exclusive pion electroproduction at high $-t$ parameterization combining Hall $\mathrm{B}$ and $\mathrm{C}$ results . . . . . . . . . . . . . . . . . . . 90 


\section{List of Tables}

2.1 Central kinematic settings used for the exclusive $\pi^{+}$electroproduction at high $-t$ measurement. . . . . . . . . . . . . . . . . 18

2.2 HMS and SOS performance specifications. . . . . . . . . . . . . . . . . . . . . . . . . .

2.3 Overview of the analysis cuts used. . . . . . . . . . . . . . . . . 40

2.4 Summary of correction factors applied to the experimental data . . . . . . . 42

2.5 Summary of the systematic uncertainties for the analysis. . . . . . . . . . 57

2.6 Unseparated cross sections for the $p\left(e, e^{\prime} \pi^{+}\right) n$ reaction at high $-t \ldots \ldots 8$

3.1 HMS and SHMS performance specifications. . . . . . . . . . . . 66

3.2 Proposed kinematic settings for E12-09-011 experiment . . . . . . . . . . . . 71

3.3 Overview of the analysis cuts used in the calculation of the rates. . . . . . . 81

3.4 Estimated singles rates for HMS and SHMS as well as real and accidental rates for the E12-09-11 experiment . . . . . . . . . . . . . . . 82

4.1 The exponential slope of of the $t$-dependence of cross sections and the interaction radii . . . . . . . . . . . . . . . . . . . . . . . 93

4.2 Aerogel indices of refraction required for upcoming experiment, E12-09-011 . 95

A.1 Updated beam time estimates for E12-09-011 experiment . . . . . . . . . . 102 


\section{Chapter 1}

\section{Introduction}

The central topic in the research area of contemporary intermediate-energy subatomic physics is the description of hadronic matter in terms of the partonic constituents (quarks, $q$, and gluons, $g$ ) of Quantum Chromodynamics (QCD), the underlying theory of the strong interaction. In particular, the interface between hadronic and partonic descriptions of the strong interaction is of primary interest since the binding and confinement of quarks and gluons into hadrons ( $q q q$, or $q \bar{q}$ objects that interact strongly) is yet to be explained by the QCD, in detail.

The $q-q$ interaction potential in QCD is dependent upon the distance of separation between the quarks and the energy of the system, while the strength of the interaction between quarks and gluons is parameterized via the energy-dependent coupling constant, $\alpha_{s}$, of the strong interaction, as shown in Figure 1.1. At higher energies corresponding to asymptotically short distance scales, typically smaller than hadron size of $\sim 1$ Fermi $\left(10^{-15} \mathrm{~m}\right)$, the strong interaction is actually weak as $\alpha_{s}$ becomes very small (see Figure 1.1), resulting in nearly free quarks and gluons inside the hadrons (i.e., the so-called "Asymptotic Freedom" where partons are not subjected to any force). The relevant degrees of freedom at short distances are in terms of quarks and gluons, collectively known as partons, and their interactions 
can be quantified very precisely using perturbative theory of QCD (pQCD).

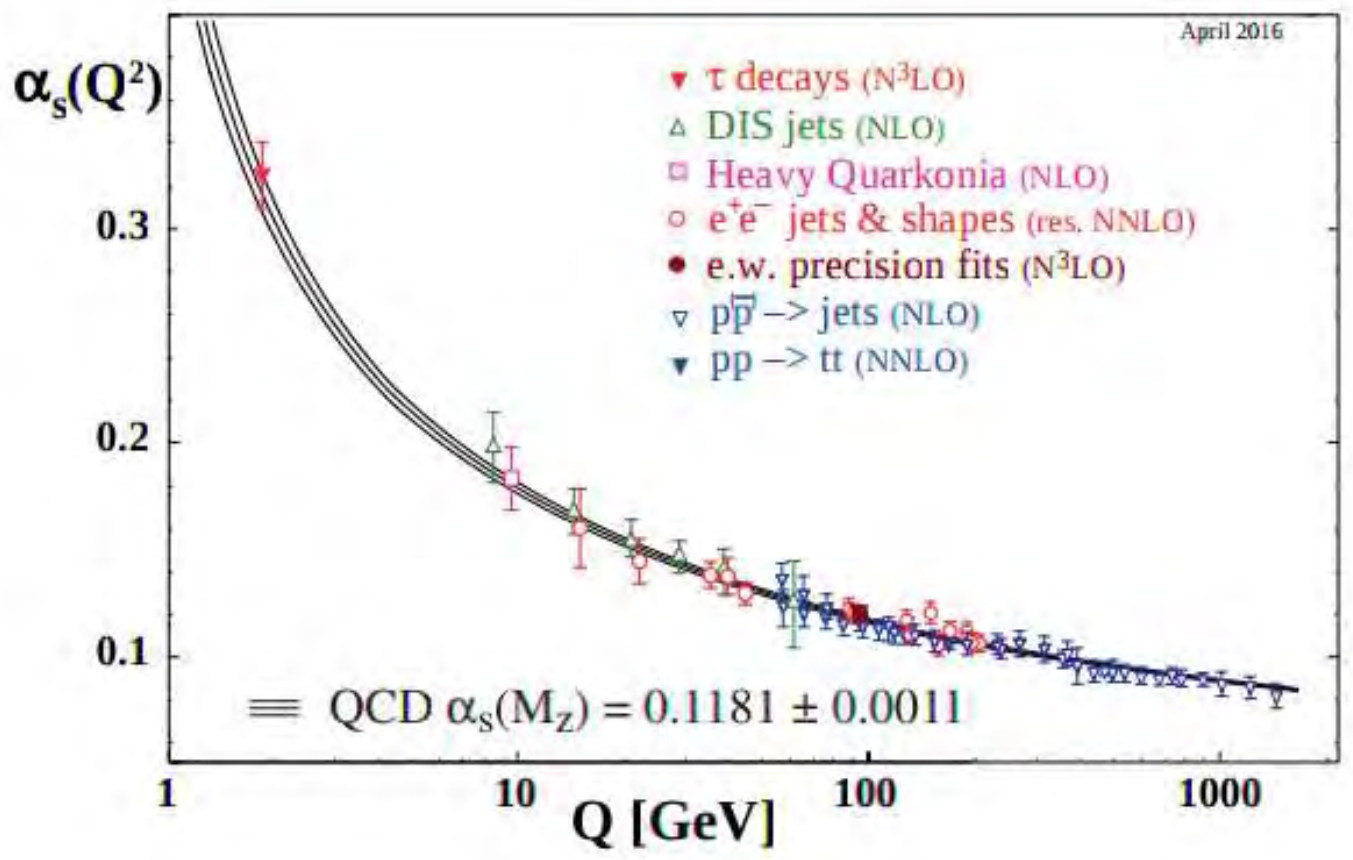

Figure 1.1: The strong coupling constant as a function of the energy scale, $Q(\mathrm{GeV})$. The wealth of available results (labeled in the figure) provides a rather precise and reasonably stable world average value of $\alpha_{s}\left(M_{Z}^{2}\right)$ (also included in the figure), as well as a clear signature and proof of the energy dependence of $\alpha_{s}$, in full agreement with the QCD prediction of Asymptotic Freedom. Figure and text taken from PDG [1].

Although static properties like magnetic moments of hadrons are taken into account by the constituent quark masses in QCD, many practical calculations involving dynamical properties (e.g, interactions of gluons and sea of quark as well as charge distributions inside hadrons) using QCD are very difficult to perform at large distance scales, mainly due to the fact that for a given $q-g$ interaction the strength of the QCD coupling exponentially grows greater than unity as the discrete energy scale is decreased. At lower energies and larger distances ( $\geq 1$ Fermi), the quarks and gluons cannot be isolated due to quark confinement, which is the phenomenon in which color charged particles ( $q$ and $g$ ) cannot be isolated singularly, and therefore cannot be observed directly. Thus, the strong interaction is characterized with effective theories, such as Quantum Hadrodynamics (QHD) [2], that consider hadrons - 
mesons $(q \bar{q})$ and baryons $(q q q)$ - as elementary particles. The interactions of these hadrons are described using Yukawa-like exchange of mesons models, whereas their properties and the strength of their interactions are phenomenologically determined via experiments, e.g., hadron-hadron scattering, photoproduction, electroproduction etc. and the use of symmetry relations (duality, crossing symmetry etc.) between various reactions.

The observable degrees of freedom (hadrons) at larger distance scales, as described earlier, are different than the constituent degrees of freedom in the QCD Lagrangian, $q$ and $g$, making up the hadronic bound states. Since the connection between these two domains of the strong interaction is not well understood, the study of transition region between partonic to hadronic degrees of freedom is of prime importance in characterizing hadrons from first principles. In order to study the transition region, a consistent analysis that includes both distance scales is required. However, in the absence of a complete solution to QCD, the predictive power of the theory is limited and we mostly rely on the extraction of related information from experimental data in the non-perturbative regime. The results thus extracted can be used to constrain effective theories describing the non-perturbative part of the strong interactions at low momentum transfer. The ultimate goal is to fully understand the onset of pQCD as the momentum transfer is increased [3].

Exclusive electroproduction of mesons, which is at the heart of this dissertation work, provides one way to study the transition from non-perturbative to perturbative regime of QCD. In exclusive meson electroproduction reactions, deep inelastic scattering of electrons is used to produce a single meson off of a nucleon (proton or neutron), which is detected in the experiment along which incoming and scattered electrons while the recoiling nucleon is observed via the reconstruction of missing mass. This work involves two electroproduction experiments at the Thomas Jefferson National Accelerator Facility (Jefferson Lab or JLab), aiming to study the transition region as well as the hadron structure. The first of the two is 
the exclusive charged pion electroproduction experiment at high four-momentum transfer, $-t$, conducted in 2003. The second is the upcoming $12 \mathrm{GeV}$ JLab-era kaon electroproduction experiment to be conducted in 2018. In this work, the analysis of E01-004 experimental data to determine $p\left(e, e^{\prime} \pi^{+}\right) n$ cross sections as well as the analysis of $p\left(e, e^{\prime} K^{+}\right) \Lambda\left(\Sigma^{0}\right)$ simulated data to project the estimated rates are presented. $\pi^{+}$and $K^{+}$are of interest since they are the two lightest mesons with relatively simple $q \bar{q}$ valence quarks structure. This thesis contains four chapters that are arranged in a manner described below.

The rest of the first chapter provides a brief introduction to deep exclusive electroproduction reactions; their relevance to QCD is also described in the same section. The first chapter also lays the theoretical ground for the determination of cross sections from the experimental data. The reaction kinematics and various kinematic quantities used in the analysis are also defined in this chapter. Finally, mathematical formalism to determine the cross section as well as to extract the structure functions from meson electroproduction data is presented towards the end of the chapter.

The second chapter is dedicated to the analysis of exclusive charged pion electroproduction data at high $-t$. The first half of the chapter is mainly concerned with the experiment, where discussions on experimental set-up, kinematics, as well as various equipments are provided. The second half of the chapter deals with the analysis of the experimental data to determine the cross sections. Here, the detailed description of the analysis of the experimental data, including the event selection, background subtraction, and yield calculation are documented along with a brief discussion on the Monte Carlo simulation tool used for the analysis. The chapter also gives a discussion on the statistical and systematic uncertainties in the determination of the cross sections and it ends with the comparison of the results to prior experimental data as well as two theoretical models.

The third chapter of this thesis focuses on the upcoming kaon electroproduction experi- 
ment, in particular, the analysis of simulated data for the projection of real and accidental coincidence rates. The goals of the experiments are covered, along with a discussion on upgraded equipments as well as the kinematic coverage of the experiment. The models used in the Monte Carlo simulation to produce the simulated data are also discussed in the first part of the chapter. The final half of the chapter mainly deals with the analysis of the simulated data to estimate the real and accidental coincidence rates. The procedure followed to project these rates are documented and the results are also provided towards the end of this chapter.

In the final chapter of this thesis, the discussion and implications of the results from the pion electroproduction experiment is provided. The chapter also provides a discussion on the results of the kaon electroproduction rates projections along with the updated beam time schedule.

\subsection{Deep Inelastic Exclusive Reactions}

In 1955, Hofstadter and McAllister showed that the observed cross sections from elastic scattering off hydrogen (proton) target deviated from the so-called "Mott" scattering crosssection, corresponding to elastic scattering between electron and a point-like nucleon target [4]. After various theoretical models were unable to explain the deviation in the two cross sections results [5], it was realized that the nucleons are not point-like particles. Rather, they are of finite size $(\sim 1$ Fermi), while the evidence from $\mathrm{SU}(3)$ symmetry, giving rise to the quark model [6], suggested that they are built from point-like quarks. Since then, the inner structure of the nucleons has garnered much attention from the hadronic physics community and deep inelastic scattering (DIS) of leptons (electron, muons and neutrinos) has become a popular probe to study nucleon structure.

The wide use of DIS of leptons, especially electrons, can be ascribed to two main reasons. Firstly, probing the nucleons dynamically using DIS of electrons has led to important out- 
comes in the field of hadronic physics, e.g., an understanding of the nucleon structure, the confirmation of the quarks as the point-like substructure of nucleon, and the measurement of the quark properties. Secondly, the leptons, having no color charge, do not involve in strong interactions and probe the inner structure of nucleon via electromagnetic (and/or weak) interactions. Since the electromagnetic processes, such as electron-photon interaction in the case of DIS of electron, can be calculated to a high precision by one of the most successful theories in modern physics, Quantum Electrodynamics (QED), the accelerated electron beam in accelerator facilities like Jefferson Lab is a simple and well understood probe, avoiding many complications, such as final state interactions that arise from the influence of the strong interaction in a technique like hadron-hadron scattering.

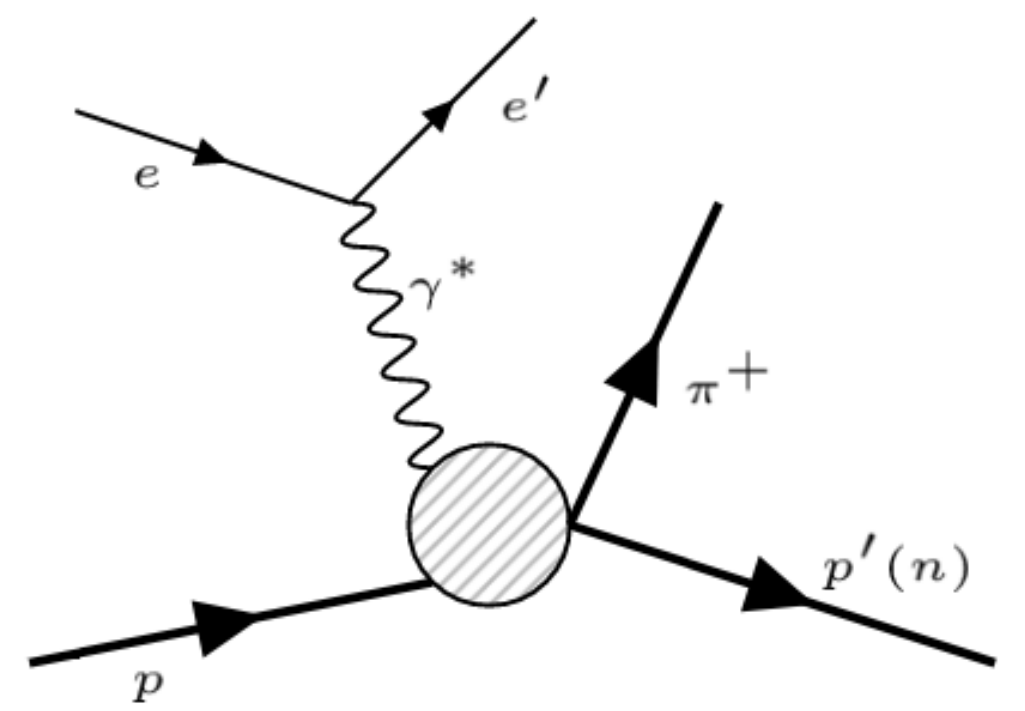

Figure 1.2: Feynman representation for the exclusive $\pi^{+}$electroproduction via deep inelastic scattering off the proton. In the diagram, an electron, $e$, is inelastically scattered off a proton, $p$, producing a space-like virtual photon, $\gamma^{*}$, which then interacts with $p$, resulting in $\pi^{+}$production. The blob in the middle represents the $\gamma^{*}-p$ interactions, while $p^{\prime}(n)$ is the final state of the nucleon, $p$, involved in the reaction.

In inclusive deep inelastic electron scattering reactions, typically only the energies and momenta of the initial and final electrons are observed, but not the particles produced from 


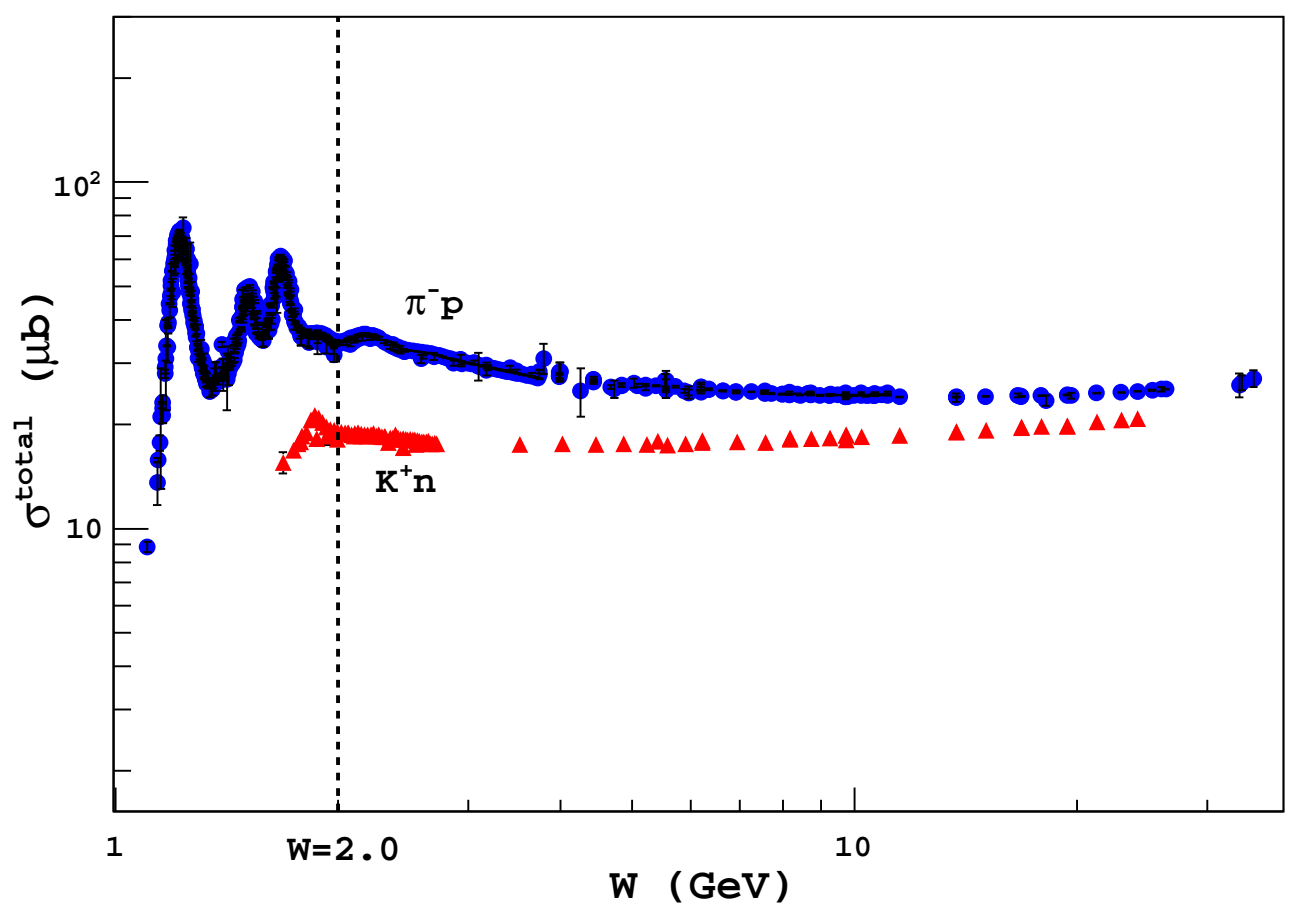

Figure 1.3: Total collision cross sections as a function of invariant mass, $W$, for $\pi^{-} p$ and $K^{+} n$ collisions (log-log plot). $\pi^{-} p$ is the charge symmetric state of the final state hadrons in exclusive $\pi^{+}$electroproduction, with respect to the $z$-component of isospin, $I_{z} \cdot K^{+} n$ denotes the total collision cross-sections. The region with peaks to the left of the dotted line at $W=2.0 \mathrm{GeV}$ is the resonance region, while the structureless flat region to the right of the dotted line is the deep inelastic scattering region [1].

the target, resulting in what is often called inclusive cross sections. However, if the energies and momenta of all produced particles are also observed, in addition to those of initial and scattered electron, such reactions are called deep inelastic exclusive reactions [7]. In Figure 1.2, a Feynman representation of the exclusive $\pi^{+}$electroproduction reaction is shown.

Depending on the kinematics, the system probed by the interaction via electron scattering can vary quite significantly. At low energy and momentum transfer, the space-like virtual photon, $\gamma^{* 1}$, interacts with the entire nucleon, scattering elastically, or exciting nucleon

\footnotetext{
${ }^{1}$ The virtual photon, $\gamma^{*}$, is divided into two types: space-like (i.e., more momentum than energy), and time-like (i.e., more energy than momentum). Space-like $\gamma^{*}$ has no real "rest mass" (it only has imaginary "rest mass") while time-like $\gamma^{*}$ does. The virtual photon used throughout this dissertation refers to the space-like $\gamma^{*}$.
} 
resonances - a special energy at which the particles involved 'prefer' to interact, forming a short-lived semibound state before breaking apart [8]. As the energy and momentum transfer increase, $\gamma^{*}$ is able to probe smaller distance scales as the system enters the deep inelastic region, and the interaction becomes more sensitive to the partonic degrees of freedom in the nucleon. In the presence of nucleon resonances, such as $N^{*}, \Delta, \Delta^{*}$, the appropriate physics of interest are the study of resonances and baryonic matter structure [9], which are different from the scope of this work, i.e., the study of hard-soft QCD transition using exclusive meson electroproduction via DIS. Thus, in order to avoid these resonances, the exclusive $\pi^{+}$and $K^{+}$ electroproduction measurements are performed in the kinematic region with the invariant effective mass, $W$, above $\sim 2 \mathrm{GeV}$, which is shown in Figure 1.3.

In order to take deep inelastic exclusive measurements off the nucleon, generally a coincidence electroproduction is performed, allowing for the properties of both the target nucleon and a produced particle to be measured. This is accomplished by probing the target nucleon with a beam of accelerated electrons, and detecting scattered probe and produced ejectile simultaneously, within a small coincidence window - typically chosen to be $<2.0 \mathrm{~ns}$ in the coincidence electroproduction experiments. The reactions describing the exclusive $\pi^{+}$and $K^{+}$electroproduction are given by

$$
e+p \rightarrow e^{\prime}+\pi^{+}+n
$$

and

$$
e+p \rightarrow e^{\prime}+K^{+}+\left(\Lambda \text { or } \Sigma^{0}\right)
$$

respectively. In the following section, the kinematics of exclusive pion electroproduction, $p\left(e, e^{\prime} \pi^{+}\right) n$, reaction are presented, along with the definitions of kinematic quantities used in the analysis. It should be noted that the kinematics of the $p\left(e, e^{\prime} K^{+}\right) \Lambda\left(\Sigma^{0}\right)$ reaction are analogous to that of the pion and are briefly discussed in Chapter 3 . 


\subsubsection{Relevant Kinematics Quantities}

The kinematics of the $\pi^{+}$electroproduction reaction in the laboratory frame are shown in Figure 1.4. The following treatment is based on Volmer [10], which has been successfully used for the measurement of exclusive pion electroproduction $[3,11,12]$. The incident electron with four momentum $k=\left(\varepsilon_{k}, \mathbf{k}\right)$ interacts with the nucleon via virtual photon, $\gamma^{*}$, exchange. The scattering plane is defined by three momentum vectors of the incoming and outgoing electrons, given by $\mathbf{k}$ and $\mathbf{k}^{\prime}$ respectively, and the electron scattering angle is denoted by $\theta_{e}$. The four-momentum transferred by the electron to the photon-nucleon system is given by $q=(\omega, \mathbf{q})$, where $\mathbf{q}=\mathbf{k}-\mathbf{k}^{\prime}$ and $\omega=E_{i}-E_{f}$. The square of the four-momentum vector $q^{2}=q_{\mu} q^{\mu}=\omega^{2}-\left|\mathbf{q}^{2}\right|$ is always negative for the space-like electron scattering process. Therefore, a Lorentz invariant quantity, $Q^{2}$, is defined, which is given by $Q^{2}=-q^{2}$. The virtual photon is absorbed by the target proton and a pion is emitted with four-momentum $p^{\prime}=\left(E\left(p^{\prime}\right), \mathbf{p}_{\pi}\right)$, where $\mathbf{p}_{\pi}$ is oriented relative to the scattering plane by a polar angle $\theta_{\pi q}$ with respect to $\mathbf{q}$, and an azimuthal angle $\phi_{\pi q}$.

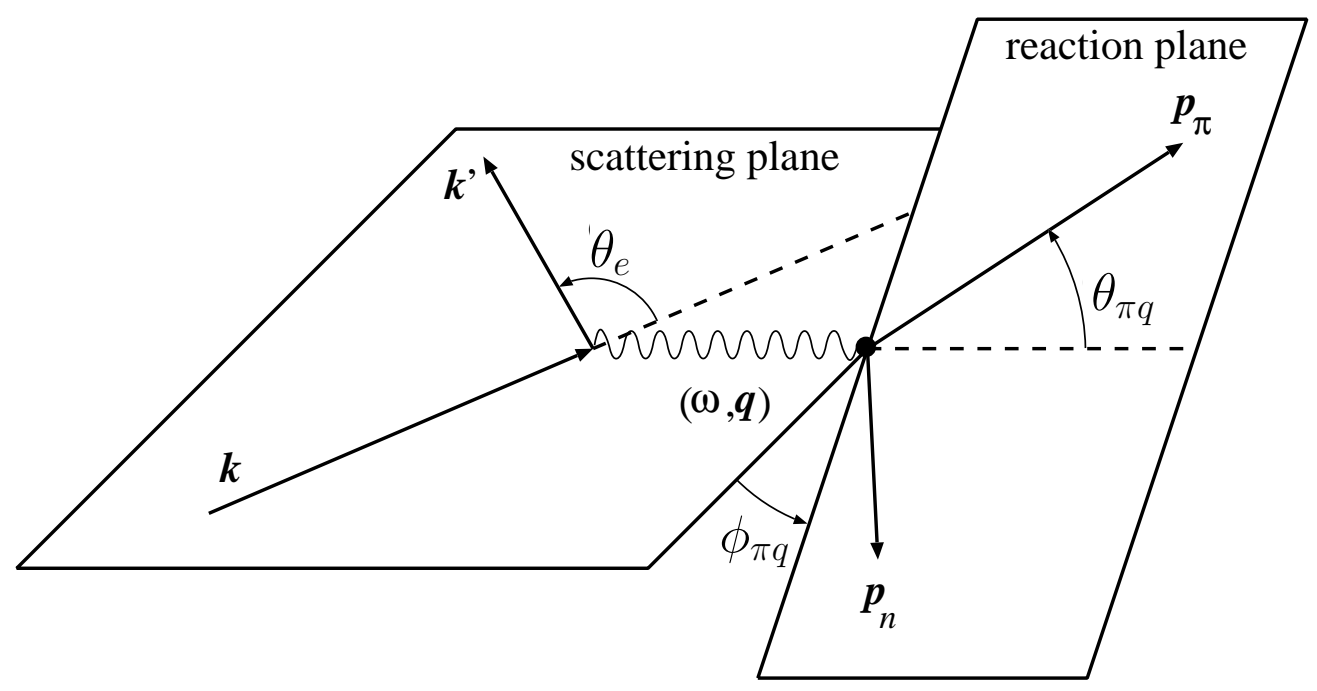

Figure 1.4: Kinematics for $p\left(e, e^{\prime} \pi^{+}\right) n$ reaction in the lab frame. The scattering plane is defined by the three-vectors of the initial and final electron. The momentum transferred to the photon-nucleon system is defined in terms of $Q^{2}$ and the reaction plane is defined by the three-vectors of the produced pion and the recoiling nucleon. 
Since the recoiled nucleon is not usually detected in the electroproduction experiment, the energy and momentum of the recoiled nucleon are reconstructed using energy and momentum conservation at the reaction vertex. For $p\left(e, e^{\prime} \pi^{+}\right) n$ reaction, the missing energy and missing momentum are given by

$$
\begin{aligned}
& E_{m}=E_{e}-E_{e^{\prime}}-E_{\pi}, \\
& \mathbf{p}_{\mathbf{m}}=\mathbf{q}-\mathbf{p}_{\pi} .
\end{aligned}
$$

Using these two quantities, the missing mass of the recoiled nucleon can be easily constructed as $M_{x}=\sqrt{E_{m}^{2}+\mathbf{p}_{\mathbf{m}}^{\mathbf{2}}}$ (further simplification also given Equation 2.6).

The kinematics of the electroproduction reaction are fully described in terms of the Lorentz invariant quantities: $W$ representing the invariant mass of the photon-nucleon system, and $t$, which is the square of the four-momentum transfer to the nucleon, in addition to $Q^{2}$. The Mandelstam variables, $s$ and $t$, for $p\left(e, e^{\prime} \pi^{+}\right) n$ reaction are defined as

$$
\begin{aligned}
& s=\left(q+p_{p}\right)^{2}=\left(p_{\pi}+p_{n}\right)^{2}, \\
& t=\left(p_{\pi}-q\right)^{2}=\left(p_{n}-p_{p}\right)^{2} .
\end{aligned}
$$

The invariant mass, $W$, is simply the square root of the Mandelstam variable, $s$, and can be expressed in terms of the mass of the proton, $M_{p}$, and the energy and four-momentum of the incoming virtual photon, $\omega$ and $Q^{2}$, respectively, using following equation.

$$
W=\sqrt{s}=\sqrt{M_{p}^{2}+2 M_{p} \omega-Q^{2}} .
$$

In the case of the exclusive pion electroproduction, the four-momentum transferred, $t$, in Equation 1.4 can be further simplified into following equation.

$$
t=\left(E_{\pi}-\omega\right)^{2}-\left|\mathbf{p}_{\pi}\right|^{2}-2\left|\mathbf{p}_{\pi}\right||\mathbf{q}| \cos \theta_{\pi q}-|\mathbf{q}|^{2}
$$

For the exclusive electroproduction reaction, $t$ is always negative, and thus, the notation $-t$ will be used henceforth. The minimum value of $-t$ will be denoted with $-t_{\text {min }}$, which is achieved when $\theta_{\pi q}=0$. Since the kinematic quantities are not independent of each other, 
$-t_{\min }$ increases with increasing values of $Q^{2}$, at fixed values of $W$.

\subsubsection{Cross Sections}

The cross sections for the exclusive electroproduction reaction can be defined using the plane wave Born approximation, where the incident and scattered electrons are described by Dirac plane waves. Similarly, the interaction with the nucleon target is described using the one-photon-exchange (OPE) approximation, where the interaction is mediated by a single virtual photon. Using both these approximations, the six-fold $\left(e, e^{\prime} \pi^{+}\right)$cross section can be written as the contraction of a lepton tensor, $L_{\mu \nu}$, and a hadron tensor, $W^{\mu \nu}[10]$.

$$
\frac{d^{6} \sigma}{d E^{\prime} d \Omega_{e^{\prime}} d \Omega_{\pi}^{*} d E_{\pi}}=\frac{\left|\mathbf{p}_{\pi}\right| E_{\pi} \alpha^{2} E^{\prime}}{Q^{4} E_{e}} L_{\mu \nu} W^{\mu \nu} .
$$

The lepton tensor can be calculated exactly using QED and the explicit structure of the hadron tensor depends on the specific process under investigation. For the exclusive meson electroproduction, as in the cases of both pions and kaons, the electroproduction cross section can be expressed as a product of a virtual photon flux factor, $\Gamma$, and a virtual photon cross section. The reduced five-fold pion electroproduction cross section is then given by,

$$
\frac{d^{5} \sigma}{d E^{\prime} d \Omega_{e^{\prime}} d \Omega_{\pi}^{*}}=\Gamma \frac{d^{2} \sigma}{d \Omega_{\pi}^{*}}
$$

where $E^{\prime}$ and $\Omega_{e^{\prime}}$ is the scattered electron lab energy and solid angle, respectively, and $\Omega_{\pi}^{*}$ is the pion solid angle in the center-of-mass frame. The virtual photon flux factor, $\Gamma$, can be expressed as:

$$
\Gamma=\frac{\alpha}{2 \pi^{2}} \frac{E^{\prime}}{E_{e}} \frac{q_{L}}{Q^{2}} \frac{1}{1-\varepsilon},
$$

where $\alpha$ is the fine structure constant $(\sim 1 / 137)$ and $\varepsilon$ describes the longitudinal polarization of the virtual photon and is given by,

$$
\varepsilon=\left(1+\frac{2|\mathbf{q}|^{2}}{Q^{2}} \tan ^{2}\left(\frac{\theta_{e}}{2}\right)\right)^{-1} .
$$


$q_{L}$ is the equivalent real photon energy, i.e., the lab energy a real photon would require to excite a target of mass, $M_{p}$, producing a system with invariant mass, $W$. It is given in the Hand convention [13] by,

$$
q_{L}=\frac{W^{2}-M_{p}^{2}}{2 M_{p}}
$$

It should be noted that a common convention for expressing the virtual photon cross section $\left(d \sigma / d \Omega_{\pi}^{*}\right)$ is in terms of the center-of-mass frame. This convention is more convenient if one wishes to extract resonance multipoles [13]. However, in this work, the virtual photon cross section will be expressed in the lab frame in the form, $d^{2} \sigma / d t d \phi$, as given in Equation 1.13 to facilitate the comparison, since various theoretical models and prior data use the same convention for expressing the cross sections. Further details are provided in Subsec. 2.3.1.

The virtual photon cross section can be decomposed into four structure functions that corresponds to the polarization states of the virtual photon: a longitudinal (L), a transverse (T), and the two interference terms (LT and TT). The two-fold differential cross section in Equation 1.8 can be expressed in terms of the structure functions as [14]:

$$
\frac{d^{2} \sigma}{d \Omega_{\pi}^{*}}=\frac{d^{2} \sigma_{T}}{d \Omega_{\pi}^{*}}+\varepsilon \frac{d^{2} \sigma_{L}}{d \Omega_{\pi}^{*}}+\sqrt{2 \varepsilon(1+\varepsilon)} \frac{d^{2} \sigma_{L T}}{d \Omega_{\pi}^{*}} \cos \phi+\varepsilon \frac{d^{2} \sigma_{T T}}{d \Omega_{\pi}^{*}} \cos 2 \phi .
$$

The differential cross section in Equation 1.12 can be expressed in the lab frame using following equation [3]:

$$
2 \pi \frac{d^{2} \sigma}{d t d \phi}=\frac{d \sigma_{T}}{d t}+\varepsilon \frac{d \sigma_{L}}{d t}+\sqrt{2 \varepsilon(1+\varepsilon)} \frac{d \sigma_{L T}}{d t} \cos \phi+\varepsilon \frac{d \sigma_{T T}}{d t} \cos 2 \phi .
$$

For the exclusive $\pi^{+}$electroproduction study at high $-t$, only the unseparated cross sections are of interest. However, for $p\left(e, e^{\prime} K^{+}\right) \Lambda\left(\Sigma^{0}\right)$ reactions, the main goal is to perform L-T-LT-TT separation of the cross sections. Along the direction of virtual photon (i.e. in parallel kinematics), the interference terms (LT and TT) can be eliminated either by taking measurements along the direction of virtual photon or by measuring these terms over the full 
$\phi$ coverage and integrating over the experimental acceptance. After the interference terms disappear, Equation 1.12 is reduced to the following form.

$$
\frac{d^{2} \sigma}{d \Omega_{\pi}^{*}}=\frac{d^{2} \sigma_{T}}{d \Omega_{\pi}^{*}}+\varepsilon \frac{d^{2} \sigma_{L}}{d \Omega_{\pi}^{*}}
$$

The longitudinal and transverse components of the cross sections can thus be separated via the so-called "Rosenbluth" technique, i.e, by taking measurements at two (or more) values of $\varepsilon$ keeping $W, Q^{2}$, and $-t$ constant and fitting a straight line to the known $\varepsilon$ dependence. The longitudinal cross section is given by the slope, while the transverse term by the $y$-intercept. 


\section{Chapter 2}

\section{Analysis of exclusive $\pi^{+}$}

\section{electroproduction data at high $-t$}

The exclusive $\pi^{+}$electroproduction data were taken as a part of experiment E01-004, $\mathrm{F}_{\pi^{-}}-2[12,11]$, with the aim to study the hard-soft transition of QCD at high four-momentum transfer, - $t$. The experiment was carried out in Jefferson Lab in 2003, and the analysis was performed to measure the unseparated cross sections over a wide $-t$ range. This chapter is devoted to the experiment and the analysis of the experimental data. It begins with an overview of the kinematic coverage of the data in Section 2.1 and provides a brief description of all the experimental apparatus used during the run period of the experiment.

Experimental cross sections are obtained by comparing the measured experimental yields to those generated by a Monte-Carlo simulation. Therefore, the data analysis of $\pi^{+}$electroproduction data over a wide $-t$ range is concerned primarily with two tasks. The first one involves deriving efficiencies corrected and charge normalized yields from the measured data: these are elaborated in Section 2.2. The second task, described in Sections 2.3-2.4, entails adjusting the Monte-Carlo simulation to generate model yields close to the measured yields in order to obtain the experimental cross sections. The latter also deals with the uncertainty 
estimates. The cross section results are presented in Section 2.5, which are then compared to the prior data $[3,15]$ as well as some theoretical models. Finally, the chapter ends with a discussion of the cross section results.

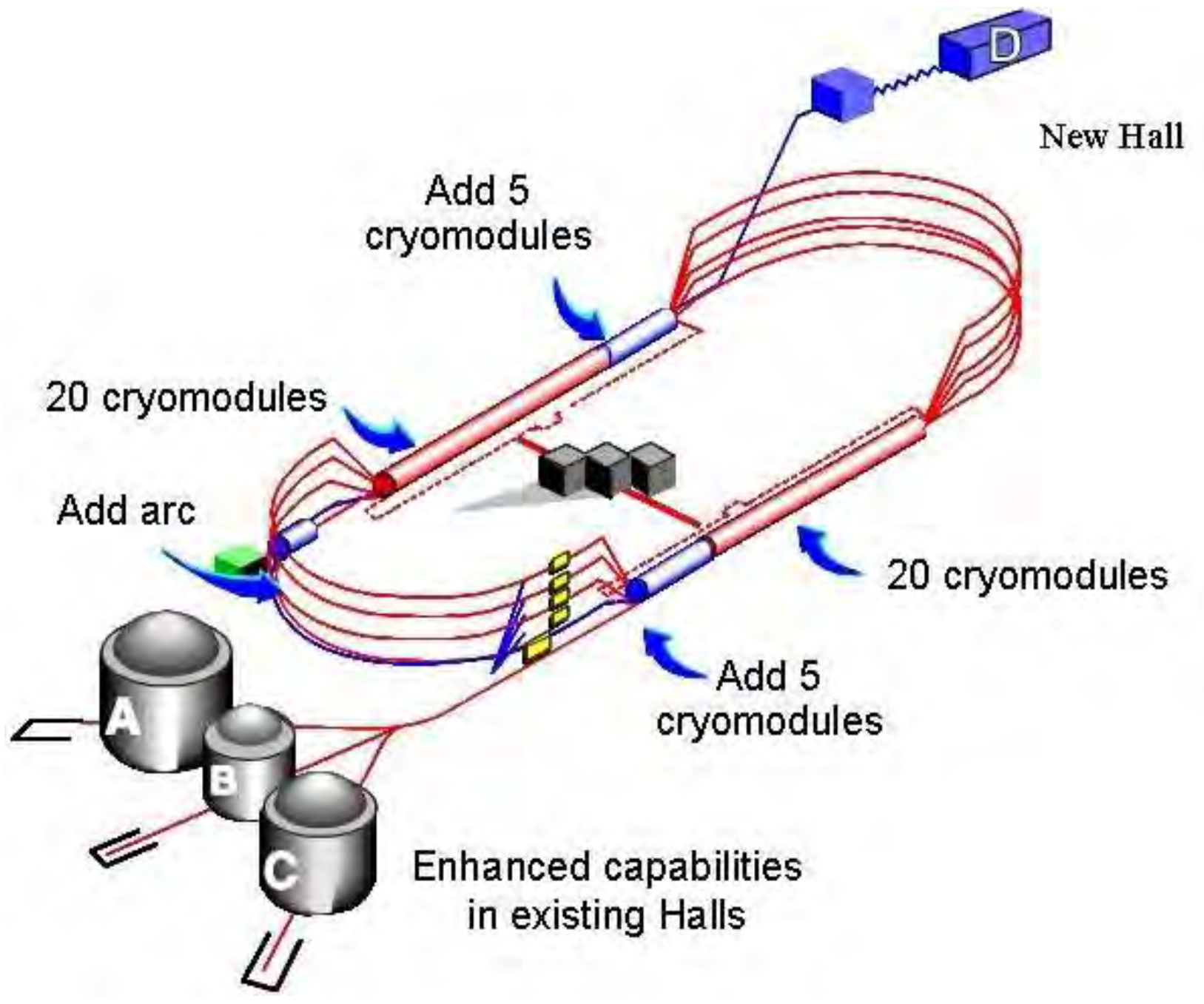

Figure 2.1: Schematic representation of the Jefferson Lab site with labels outlining the newly upgraded components [16]. (Color Online)

\section{$2.1 \quad$ Experiment and Setup}

Jefferson Lab is an intermediate energy nuclear physics facility located in Newport News, VA, USA. In 2003, Jefferson Lab consisted of a continuous wave electron accelerator and three experimental halls (A, B, and C) to study different aspects of nuclear and hadronic physics. 
Figure 2.1 shows schematic representation of Jefferson Lab. In 2014, the lab completed the upgrade shown in Figure 2.1, adding a new experimental hall, Hall D, and upgrading its electron accelerator to provide electron beam with higher energy of up to $12 \mathrm{GeV}$.

The high $-t$ data were acquired in Hall $\mathrm{C}$ as a part of the $\mathrm{F}_{\pi^{-}}-2$ experiment. The unpolarized electron beam from the Continuous Electron Beam Accelerator Facility (CEBAF) was incident on a $4 \mathrm{~cm}$ liquid hydrogen $\left(\mathrm{LH}_{2}\right)$ target located under vacuum in a scattering chamber [10]. The measurement of exclusive $\pi^{+}$electroproduction at high $-t$ was done at a fixed beam energy of $4.709 \mathrm{GeV}$, with a beam current of $75 \mu \mathrm{A}$. Two moderate acceptance, magnetic focusing spectrometers, namely, HMS and SOS, were employed to detect the particles of interest. The scattered electrons were detected in SOS, while the coincident electroproduced charged pions were detected in HMS.

\subsubsection{Experiment Kinematics}

The kinematics of the $p\left(e, e^{\prime} \pi^{+}\right) n$ reaction are illustrated in Figure 1.4 of the first chapter, along with the definitions of all the relevant kinematic variables. The central kinematic settings used for this experiment are shown in Table 2.1. The choice of kinematics for the measurement was primarily based on maximizing the $Q^{2}$ range for a value of invariant mass, $W$ above the resonance region, where constraints on the kinematics were imposed by the maximum available beam energy as well as the maximum central momentum of the SOS. The experiment covers the $t$-range from 0.272 to $2.127 \mathrm{GeV}^{2}$. The measurement was performed at fixed virtuality of the incoming photon $\left(Q^{2}\right)$ of $2.50 \mathrm{GeV}^{2}$, as well as the invariant mass $(W)$ of $2.00 \mathrm{GeV}$. In addition, the scattered electron energy, $T_{e^{\prime}}$, and central SOS angle, $\theta_{e^{\prime}}$, were also kept the same throughout the experiment.

In order to study the $t$-dependence of the exclusive pion electroproduction unseparated cross section, the central momenta of the pion arm were varied from $2.845 \mathrm{GeV} / c$ at the 


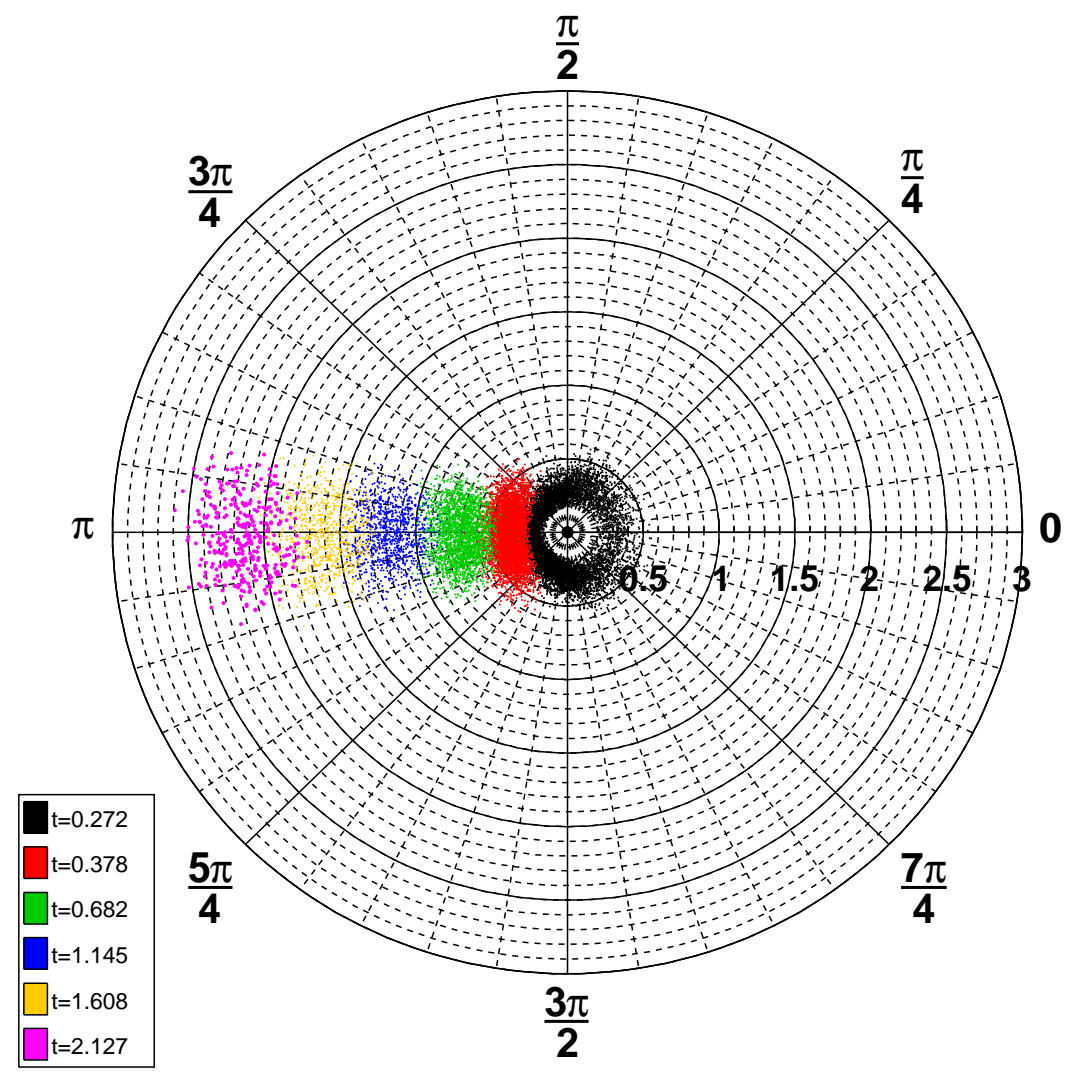

Figure 2.2: Polar plot showing the $|t|-\phi_{\pi}$ phase space coverage of the experiment. The Mandelstam variable $-t$ is plotted as the radial component and different $-t$ settings are color-coded in the plot. Similarly, $\phi_{\pi}$ is plotted as polar component that progresses counterclockwise, with $\phi_{\pi}=0^{\circ}$ at the right.

lowest $-t$ setting of $0.272 \mathrm{GeV}^{2}$ to $1.853 \mathrm{GeV} / c$ at the highest $-t$ setting of $2.127 \mathrm{GeV}^{2}$. This corresponds to varying the scattering angle, $\theta_{\pi}$, of the pion arm, which spans from $15.68^{\circ}$ at the near-parallel $\left(\theta_{\pi q} \approx 0^{\circ}\right)$ kinematics to $39.50^{\circ}$ at the highest $-t$ setting. At non-parallel kinematics, the acceptances of the two spectrometers do not provide uniform coverage in $\phi_{\pi}$. Thus, typically additional data are taken with the hadron arm, HMS, at a slightly smaller and a larger angle than the central angle to attain full $\phi_{\pi}$ coverage at a given $\theta_{\pi q}$. This is essential to the L-T separation experiments, such as E12-09-011, since the full coverage of azimuthal angle $(\phi)$ is required to extract $\sigma_{L T}$ and $\sigma_{T T}$, using the measured $\phi$ dependence of the cross section. However, for our measurement of exclusive $\pi^{+}$electroproduction at 
high $-t$, only the near-parallel setting has, approximately, full $\phi_{\pi}$ coverage; the rest of the settings have a limited $\phi_{\pi}$ span. The complete $|t|-\phi_{\pi}$ coverage of our data is illustrated in Figure 2.2.

\begin{tabular}{cccccccc}
\hline $\begin{array}{c}Q^{2} \\
\left(\mathrm{GeV}^{2}\right)\end{array}$ & $\begin{array}{c}W \\
(\mathrm{GeV})\end{array}$ & $\begin{array}{c}|t| \\
\left(\mathrm{GeV}^{2}\right)\end{array}$ & $\begin{array}{c}T_{\text {inc }} \\
(\mathrm{GeV})\end{array}$ & $\begin{array}{c}T_{e^{\prime}} \\
(\mathrm{GeV})\end{array}$ & $\begin{array}{c}P_{\pi} \\
(\mathrm{GeV} / c)\end{array}$ & $\begin{array}{c}\theta_{e} \\
(\mathrm{deg})\end{array}$ & $\begin{array}{c}\theta_{\pi} \\
(\mathrm{deg})\end{array}$ \\
\hline \hline & & 0.272 & & & 2.845 & & 15.68 \\
& & 0.378 & & & 2.788 & & 20.32 \\
2.50 & \multirow{2}{*}{2.00} & 0.688 & \multirow{2}{*}{4.70} & \multirow{2}{*}{1.74} & 2.622 & 32.40 & 25.15 \\
& & 1.145 & & & 2.378 & & 30.07 \\
& 1.608 & & & 2.131 & & 34.50 \\
& 2.127 & & & 1.853 & & 39.50 \\
\hline \hline
\end{tabular}

Table 2.1: Central kinematic settings used for the exclusive $\pi^{+}$electroproduction at high $-t$ measurement. $T$ represents the total kinetic energy and the columns with kinematic quantities that are kept constant throughout the measurement contains single value in the table.

The goal of this measurement is to determine the unseparated cross section over a range of $-t$, with the HMS+SOS spectrometers capable of measurements at high luminosity and small systematic errors. To study the $t$-dependence, the unseparated $\pi^{+}$electroproduction cross section is measured at a constant average $\phi_{\pi}$ of $\sim \pi$ and a fixed polarization of the virtual photon, $\varepsilon$, of $\sim 0.56$. This is because of the limited azimuthal acceptance of our data, which is also a limitation of our experimental procedure. Using these average values from our data, as well as Equation 1.13 for the Rosenbluth separation, the contribution from the interference terms: $\sigma_{L T}$ and $\sigma_{T T}$ can be estimated. The L-T separated cross sections from $\mathrm{F}_{\pi}-2$ experiment [11] at nominal $Q^{2}$ and $W$ of $2.45 \mathrm{GeV}^{2}$ and $2.22 \mathrm{GeV}$ (close to our kinematics region) were used to estimate the contribution from the interference terms. For average $-t$ ranging from 0.145 to $0.288 \mathrm{GeV}^{2}$, the contribution from the interference terms to the unseparated cross sections, estimated using the $\mathrm{F}_{\pi}-2$ data is small: $<7 \%$. Our data at high $-t$ will also be analyzed under different $\sigma_{L T}$ and $\sigma_{T T}$ assumptions and the results will be compared with the Hall B data [15] which has full $\phi_{\pi}$ coverage but lower statistics. 
This will discussed in more detail in Section 2.5.

\subsubsection{Accelerator}

The experiment made use of the unpolarized, continuous wave (CW, 100\% duty factor) electron beam provided by CEBAF. The high intensity, superconducting radio frequency (RF) electron accelerator at Jefferson Lab delivers an adjustable beam current up to a total of $200 \mu \mathrm{A}$ to all the experimental halls simultaneously. As shown in Figure 2.1, the accelerator consists of an injector to accelerate electrons and a Beam Switch Yard (BSY) to direct the beam towards the experimental halls. It has a loop configuration of a pair of superconducting linear accelerators (linacs) interconnected through 9 recirculation arcs. The electrons are injected into the North linac at $67 \mathrm{MeV}$ and undergo between one to five "passes" through the linacs and the arcs, in which they gain $570 \mathrm{MeV}$ per linac, or 1140 $\mathrm{MeV}$ per pass. At the time of experiment running, the accelerator was capable of delivering a maximum beam energy of $5.767 \mathrm{GeV}$ [17]. The details of the linacs and accelerator operations are discussed in a more comprehensive manner in Refs $[16,18,19]$. In 2013, the accelerator has been upgraded to $2.2 \mathrm{GeV} /$ pass $(1.1 \mathrm{GeV} /$ linac) and is now able to provide maximum beam energy of $12 \mathrm{GeV}$ (11 GeV for Halls A, B, and C). A more detailed description of the upgrade can be found in Ref. [16].

The accelerator produces short beam bunches that are $1.67 \mathrm{ps}$ long at an operating frequency of $1497 \mathrm{MHz}$. These bunches were, in turn, delivered to each of the three experimental halls simultaneously, resulting in a 2 ns bunch structure in each hall. This intrinsic micro-structure of the beam helps in the identification of coincident events, which is further described in the next section. The beam position and angle on the target were monitored using beam position monitors (BPMs) in order to precisely determine the kinematics of the experiment. The position measurement was performed with an accuracy of about $0.5 \mathrm{~mm}$, 
while the accuracy of the incident angle was about $0.2 \mathrm{mrad}$. The beam current was measured using two beam current monitors (BCM1 and BCM2) that are described thoroughly in Ref. [20]. To minimize drifts in gain, an Unser current monitor [21] was used to calibrate both of the BCMs. The Unser current monitor has an extremely stable gain, but suffers from large drifts in the offset on short time scales. The total uncertainty for the charge measurement was $0.5 \%$, out of which $0.2 \%$ is the run-to-run uncertainty in the current as measured by BCMs and $0.3 \%$ is the normalization uncertainty from the Unser monitor [20, 21].

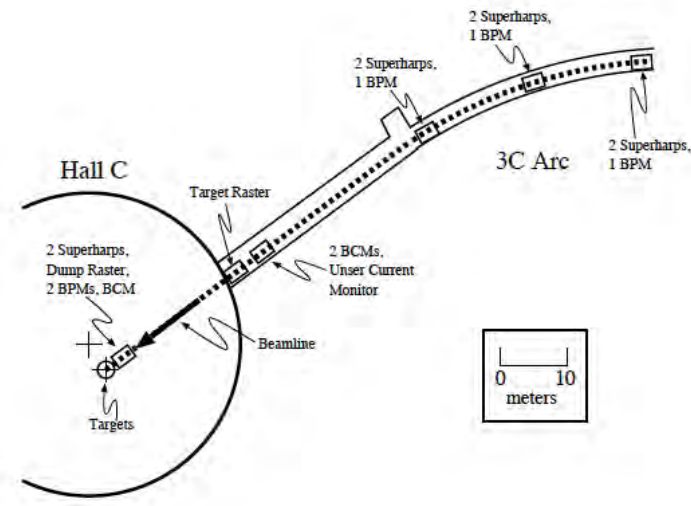

(a) Hall C Arc

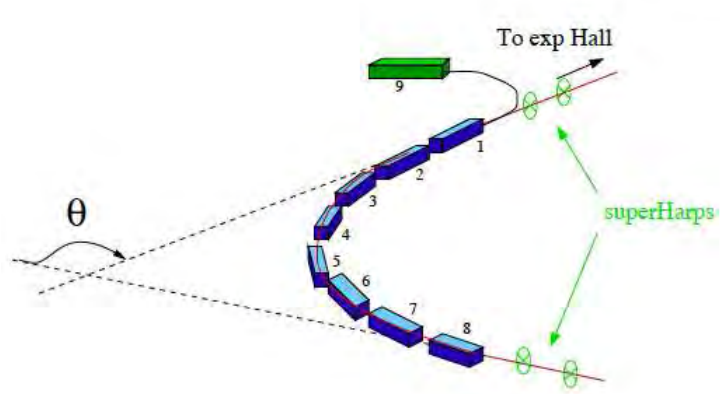

(b) Arc Energy Measurement

Figure 2.3: Beam Line Elements. The arc is used as a spectrometer to perform beam energy measurement. Only the dipole magnets are energized to bend the beam while the other magnets are degaussed to minimize the residual fields. With known magnetic field given by $\int B \cdot d l$, the beam energy can be calculated using the equation: $p=\frac{e}{\theta_{\text {arc }}} \int B \cdot d l$, where $p$ is the particle momentum, $e$ is the electron charge and $\theta_{\text {arc }}$ is the bend angle of the arc [3].

The energy of the electron beam in Hall $\mathrm{C}$ is measured using the arc method. The measurement was performed by deflecting the electron beam in a known magnetic $(\vec{B})$ field in the Hall $\mathrm{C}$ arc, as shown in Figure 2.3. It makes use of the fact that an electron moves on a circular trajectory in a constant $\vec{B}$ field where its radius depends on the strength of the $\vec{B}$ field and the electron momentum. Including the uncertainty in the field integral and the angular uncertainty, the beam energy can be determined with a precision of $\frac{\delta p}{p} \approx 1 \times 10^{-4}$. A detailed description of the arc method can be found in Ref. [22]. 


\subsubsection{Cryogenic Target}

The target system in Hall $\mathrm{C}$ consists of a three-loop cryogenic target stack, mounted together with a special optics target assembly on a target ladder in the scattering chamber. The solid target ladder consists of five carbon and two aluminum foils at different $z$-positions in the beam direction. The solid carbon foils, positioned at $z=0, \pm 3.8, \pm 7.5 \mathrm{~cm}$, are used to calibrate the spectrometer optics properties. Similarly, the two aluminum foils, placed 4 cm apart from each other, constitute the "dummy" target, which were used to measure the contribution of the aluminum cell wall to the cryotarget yields. Both the scattering chamber and the cryotarget system are standard Hall $\mathrm{C}$ equipment and are extensively discussed in Refs. [23, 24].

For the experiment, liquid hydrogen $\left(\mathrm{LH}_{2}\right)$ was used as the proton target. The $\mathrm{LH}_{2}$ target is a $4.0 \mathrm{~cm}$ diameter cylindrical cell with vertical axis (vertical-flow "tuna can" design). Along the direction of the beam, the target is $4.0 \mathrm{~cm}$ long and its $0.0127 \mathrm{~cm}$ thick cell walls are made from aluminum alloy Al-T6061 [23]; to speed up the data acquisition, higher strength Al-T7075 alloy, which is $\sim 7.8$ times thicker than the cryotarget cell walls, are used as the dummy target at room temperature (see Subsec. 2.2.3). Helium at $15 \mathrm{~K}$ provided cooling for the cryotarget, which is typically kept at a nominal operating temperature about $2 \mathrm{~K}$ below the hydrogen boiling point. Thus, the $\mathrm{LH}_{2}$ target was kept at a temperature of $19 \mathrm{~K}$ and a pressure of $166 \mathrm{kPa}$, giving a density of $0.0723 \pm 0.0005 \mathrm{~g} / \mathrm{cm}^{3}$. The uncertainty in the density is completely due to the equation of state. More details on both cryogenic and dummy targets can be found in Ref. [25].

\subsubsection{Spectrometers}

The core of the Jefferson Lab Hall C experimental apparatus consists of two medium resolution magnetic spectrometers that are used for the detection of the particles of interest. The 
experiment made use of the standard Hall C spectrometers: High Momentum Spectrometer (HMS) and Short Orbit Spectrometer (SOS), to measure the exclusive $\pi^{+}$electroproduction cross section. Since the data were taken in 2003, Hall C, like the CEBAF, went through an upgrade process, which mainly involved replacing the old SOS with another high momentum spectrometer, the Super High Momentum Spectrometer (SHMS). The upgrade is in its final stage now, with calibration of the detectors already underway. The new SHMS will be closely examined in Chapter 3 (Section 3.1).

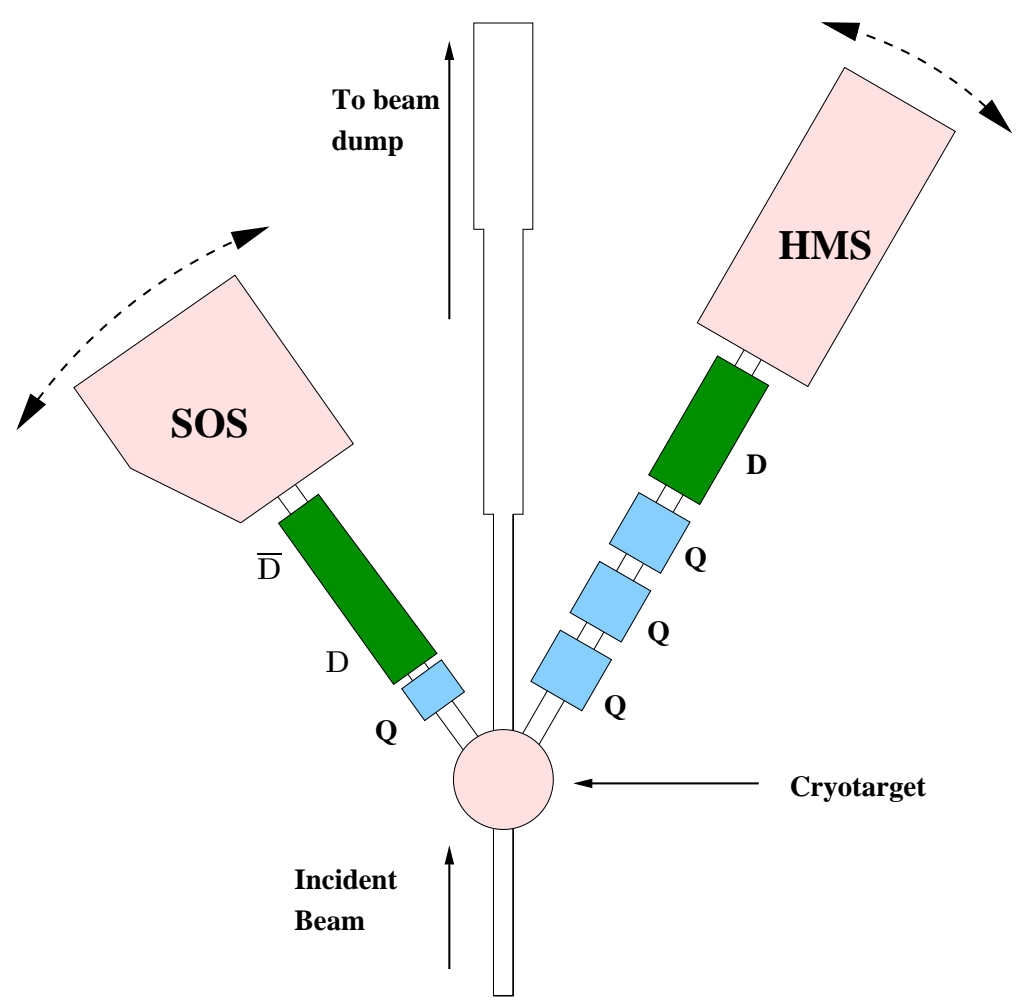

Figure 2.4: Schematic top view of the Jefferson Lab Hall C, showing HMS and SOS relative to the target and the beamline.

A schematic aerial view of the Hall $\mathrm{C}$ spectrometers during the experiment is shown in Figure 2.4. Both HMS and SOS have relatively large momentum and solid angle acceptances, as shown in Table 2.2. Additionally, they are also equipped with similar and highly versatile detector packages (see Figure 2.5) so that they can be used as an electron or a hadron detector. However, the design concepts of these two spectrometers are different. SOS has 
relatively short flight path of about $7.4 \mathrm{~m}$ and was optimized for the detection of short-lived particles at the expense of its maximum momentum, which is about $1.75 \mathrm{GeV} / c$. On the other hand, HMS, with its longer path length of $26 \mathrm{~m}$, is capable of detecting electrons or hadrons with momenta as high as $7.50 \mathrm{GeV} / c$. During the experiment, HMS was used to detect the charged pions even though the SOS is ideally suited to detect short-lived particles such as pions and kaons, because the momenta of the electroproduced pions exceeded the maximum central momentum of the SOS.

\section{High Momentum Spectrometer (HMS)}

The HMS is a long focal length, $25^{\circ}$ vertical bend focusing spectrometer. It consists of four super-conducting magnets, which include three focusing quadrupoles $(Q 1, Q 2, Q 3)$ and one momentum analyzing dipole $(d)$ with the focus perpendicular to the dispersive direction. They operate in a point-to-point tune for the central ray and provide momentum acceptance of about $\pm 10 \%$. Detectors are housed in a concrete shielding hut $26 \mathrm{~m}$ from the spectrometer pivot. The detector hut, as well as all the magnets, are mounted on a common carriage, which can be moved on rails around a rigidly mounted central bearing. The design specification comparing HMS to SOS can be found in Table 2.2. For a detailed description of the spectrometer hardware, refer to Ref. [26].

To minimize multiple scattering and to provide thermal insulation, the region between the first quadrupole magnet $(Q 1)$ and the entrance to the spectrometer hut from the target chamber was evacuated. The vacuum region is separated from the surrounding environment by $0.508 \mathrm{~mm}$ thick titanium vacuum windows (radiation length of $3.56 \mathrm{~cm}$ ) [11]. This window was installed in Hall $\mathrm{C}$ prior to the experiment, replacing the standard mylar window. A detailed discussion of the Hall $\mathrm{C}$ spectrometer vacuum system and vacuum windows can also be found in Ref. [26]. Similarly, a collimator was positioned in a collimator box between the 
target and $Q 1$ to study the HMS angular acceptance. The collimator, designed from 3.175 cm thick HEAVYMET (machinable tungsten alloy with $10 \% \mathrm{CuNi}$ ), defines a maximum HMS solid angle acceptance of 6.8 msr. More details on the collimator can be found in various Hall C theses [3, 10, 25].

\begin{tabular}{lcr}
\hline \multicolumn{1}{c}{ Quantity } & SOS & HMS \\
\hline \hline Central Momenta Range $(\mathrm{GeV} / \mathrm{c})$ & 1.75 & $0.5-7.5$ \\
Focal Length $(\mathrm{m})$ & 7.4 & 26.1 \\
Scattering Angle Range $\left(^{\circ}\right)$ & $13.4-165$ & $10.5-85$ \\
Momentum Acceptance & $\pm 20 \%$ & $\pm 10 \%$ \\
Momentum Resolution & $0.1 \%$ & $<0.1 \%$ \\
Solid Angle Acceptance (msr) & 7.5 & 6.8 \\
Horizontal Acceptance (mrad) & \pm 57.5 & \pm 27.5 \\
Vertical Acceptance (mrad) & \pm 37.5 & \pm 70.0 \\
Horizontal Resolution (mrad) & 2.5 & 1.0 \\
Vertical Resolution $(\mathrm{mrad})$ & 0.5 & 2.0 \\
Maximum DAQ Rate & $\sim 2000$ events/second & $\sim 2000$ events/second \\
Target Vertex Length $(\mathrm{cm})$ & \pm 1.5 & \pm 7.0 \\
Target Vertex Reconstruction Accuracy $(\mathrm{mm})$ & 1.0 & 1.0 \\
\hline \hline
\end{tabular}

Table 2.2: The performance and design parameters comparison between HMS and SOS [16].

For both spectrometers, the momentum is defined by the magnetic field integral over the central trajectory. In order to set the HMS momentum in a reproducible manner, an NMR probe was used in the dipole to fix the dipole field, with a reproducibility of the magnetic field at the level of one part in $10^{4}$ and a stability to within one part in $10^{5}$. Likewise, to ensure the reproducibility of the quadrupole fields, the quadrupoles are set by current using special procedure described in Refs. [10, 13], and are monitored using the power supply readback current and Hall probes.

\section{Short Orbit Spectrometer (SOS)}

The SOS is a replica of the Medium Resolution Spectrometer at the Los Alamos Meson Physics Facility (LAMPF) [18]. It consists of a $Q D \bar{D}$ configuration for the magnets, where 
$Q$ focuses the charged particles in the non-dispersive direction, $D$ bends them $33^{\circ}$ upward and $\bar{D}$ bends them downward by $15^{\circ}$. In addition to the $Q$, the fringe field due to the curved shape of the ends of the dipole magnets also provide additional focusing of the particles into the detector hut. Unlike HMS, these magnets are non-superconducting. They are watercooled and rest on a common carriage arm. A collimator box, analogous to the HMS, is placed in front of the quadrupole to measure the angular acceptance of the spectrometer. The design specifications of the SOS are listed in Table 2.2.

To determine the central momentum of the spectrometer, the SOS magnets are set by field, measured with Hall probe, providing a short-term reproducibility of $\pm 15 \mathrm{G}$ and longterm drifts of only a few parts in $10^{4}$. A particular cycling procedure, as described in Ref. [3], was used to make sure that the SOS magnets lie on the same part of the B-I hysteresis curve. Due to the iron in the magnets, the magnets in the SOS have some saturation effects at the regions of high fields corresponding to momenta higher than $1.0 \mathrm{GeV} / c$. At the maximum SOS momentum of $1.75 \mathrm{GeV} / c$, the deviation of the true central momentum of the spectrometer is in the order of $0.6 \%$. Thus, a correction to the central momentum had to be applied, which is detailed in Ref. [3].

\subsubsection{Detector Packages}

Both the HMS and SOS make use of similar detector packages, located in the detector huts of the respective spectrometers. The detector packages are standard Hall C equipment and their similarity in both spectrometers is important to ensure that the HMS and SOS can be used as hadron or electron arm interchangeably. The stack consists of two horizontal drift chambers for particle trajectory reconstruction, four scintillator hodoscopes for trigger set-up, and a gas ČCerenkov detector (see Figure 2.5) and a segmented electromagnetic leadglass calorimeter for particle identification. For the $\mathrm{F}_{\pi^{-}}-2$ experiment, an aerogel Cerenkov 
was added to the HMS detector with an aim of improving the pion-proton separation. A thorough description of all the detector components can be found in Refs. [20, 23, 26]

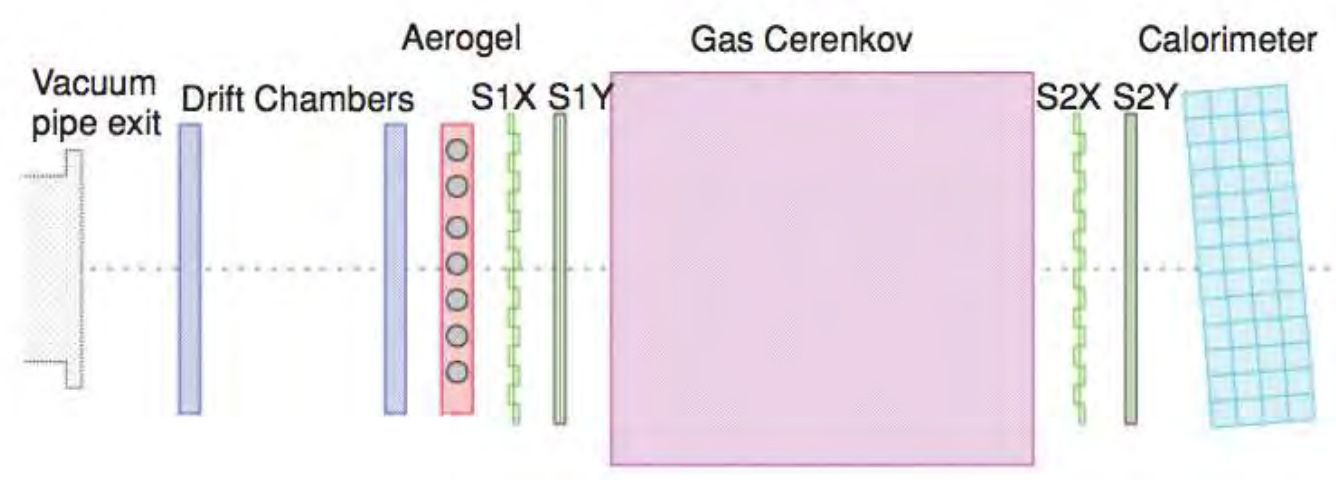

Figure 2.5: Schematic side view of the HMS detector packages during the pion electroproduction at high $-t$ run period.

\section{Drift Chambers}

The detector packages of both spectrometers contain a pair of drift chambers for tracking the trajectory of charged particles traversing through the respective spectrometers. The basic operating principle is based on ionization of inert gases induced by a charged particle [3]. Each drift chamber consists of six planes of sense wires that are $1 \mathrm{~cm}$ apart in spacing. The drift chambers in the SOS have six planes in the order: $u, u^{\prime}, x, x^{\prime}, v, v^{\prime}$, where the sense wires are oriented in three different directions. Similarly, the HMS drift chambers planes are ordered $x, y, u, v, y^{\prime}, x^{\prime}$ and the sense wires in the HMS are oriented in different directions. The orientation of wires in multiple directions allows for the measurement of the $x$ and $y$ hit positions of an incident charged particles. The $x$ and $y$ planes provide the vertical and horizontal track position, respectively while $u$ and $v$ planes are rotated by $\pm 15^{\circ}$ for the HMS and $\pm 60^{\circ}$ for the SOS, with respect to the $x$ plane. The redundancies in the number of planes used in the chambers are helpful in resolving multiple hits ambiguity and 
"left-right" ambiguity (i.e. which side of a wire a particle passed) [11]. Moreover, they also help determine a single-chamber estimate of the particle track. Further information on the workings of the drift chambers are detailed in Ref. [27].

\section{Hodoscopes}

Hodoscopes in both spectrometers consist of two scintillator planes and are situated before and after the threshold gas C̆erenkov counters. When charged particles passing through the scintillator material ionize electrons in the medium, the molecules get excited to higher energy levels. The de-excitation of these molecules emit photons, which are read out by photomultiplier tubes (PMTs) at both ends. In addition to facilitating the time-of-flight measurement, the hodoscopes are also an integral part of the trigger system in Hall C. In the SOS, the first plane of each hodoscope is segmented into "paddles" in the horizontal direction; the second plane in the vertical direction. The order of the planes is opposite for the HMS. The dimensions of paddles and geometry of the hodoscopes are discussed in Ref. [3], whereas the use of signals from hodoscopes in the trigger system is discussed in the end of this section.

\section{Čerenkov Detectors}

The primary purpose of the threshold gas Čerenkov detectors in both HMS and SOS is the separation of pions and electrons. These C̆erenkov detectors use the particle dependent threshold of Cerenkov radiation when a particle passes through a given medium in order to separate charged particles. More details on the working principle of Čerenkov detectors are given in Ref. [28]. The SOS gas Čerenkov detector is a cylindrical tank consisting four parabolic mirrors and four PMTs, maintained at atmospheric pressure with Freon-12 $\left(\mathrm{CCl}_{2} \mathrm{~F}_{2}\right)$. It has a refractive index of 1.00108 , which yields a pion threshold well above the 
maximum SOS central momentum, of $3 \mathrm{GeV} / c$.

The HMS detector stack at the time of data taking, on the other hand, contained two $\breve{C}$ Cerenkov detectors. Along with a gas C̆erenkov detector for pion-electron separation, the HMS was also equipped with an aerogel Čerenkov detector in order to separate pions and protons above momenta of $3 \mathrm{GeV} / c$. This was necessary because of the limitation on the time-of-flight resolution requirement needed to separate high momentum pions and protons [29]. The aerogel Cerenkov detector was added in 2003 for the $\mathrm{F}_{\pi^{-}}-2$ experiment and a refractive index of 1.03 was used, giving a pion threshold of $0.57 \mathrm{GeV} / c$ and a proton threshold of 3.8 GeV/c. The gas Cerenkov detector in the HMS is also a cylindrical tank, however unlike the SOS one, it has two parabolic mirrors as well as two PMTs mounted on the top and bottom surfaces. It was filled with $\mathrm{C}_{4} \mathrm{~F}_{10}$ gas at $47 \mathrm{kPa}$. It has refractive index of 1.00066 at that pressure, providing electron thresholds below $10 \mathrm{MeV} / c$ and pion thresholds of 3.8 $\mathrm{GeV} / c$. Further details on the C̆erenkov detectors used for the experiments can be found in Refs. [29, 30].

\section{Lead-glass Calorimeter}

The lead-glass calorimeter, located at the back of detector hut for both spectrometers, provides an additional means of selecting as well as separating pions and electrons. The principle of particle detection using calorimeters is based on the production of an electromagnetic shower in the lead glass material and the consequent Bremsstrahlung process. It has $10 \times 10 \times 70 \mathrm{~cm}^{3}$ blocks arranged in four different planes, stacked 13 and 11 blocks high in the HMS and SOS, respectively. Keeping in mind the losses due to particles passing through gaps between the blocks, the entire detector is tilted $5^{\circ}$ (refer to Figure 2.5) with respect to the central ray of the spectrometer. Moreover, each block is wrapped with aluminized mylar and Tedlar to ensure light-tightness. The calorimeter system hardware for both HMS and 
SOS are explained in more thoroughly in Ref. [26].

\subsubsection{Trigger System and Data Acquisition}

One of the main components of the Jefferson Lab Hall C data acquisition (DAQ) system is the trigger system, which is necessary to limit the large rate of particles from overwhelming the DAQ system. The main purpose of a hardware trigger system is to reduce electronic deadtime and total data influx while also providing a high trigger efficiency. This is achieved by the formation of a combination of logic signals that indicate when a particular set of detectors fired. The trigger system in Hall $\mathrm{C}$ has two main elements: single-arm trigger and coincidence trigger. The singles trigger can be further divided into two components: one coming out of the respective hodoscopes and the other from the combination of the signals from the gas C̆erenkov and the calorimeter. If these single-arm trigger signals are within a particular coincidence window, typically $40 \mathrm{~ns}$, then a coincidence trigger is formed and the corresponding event is recorded by the data acquisition system.

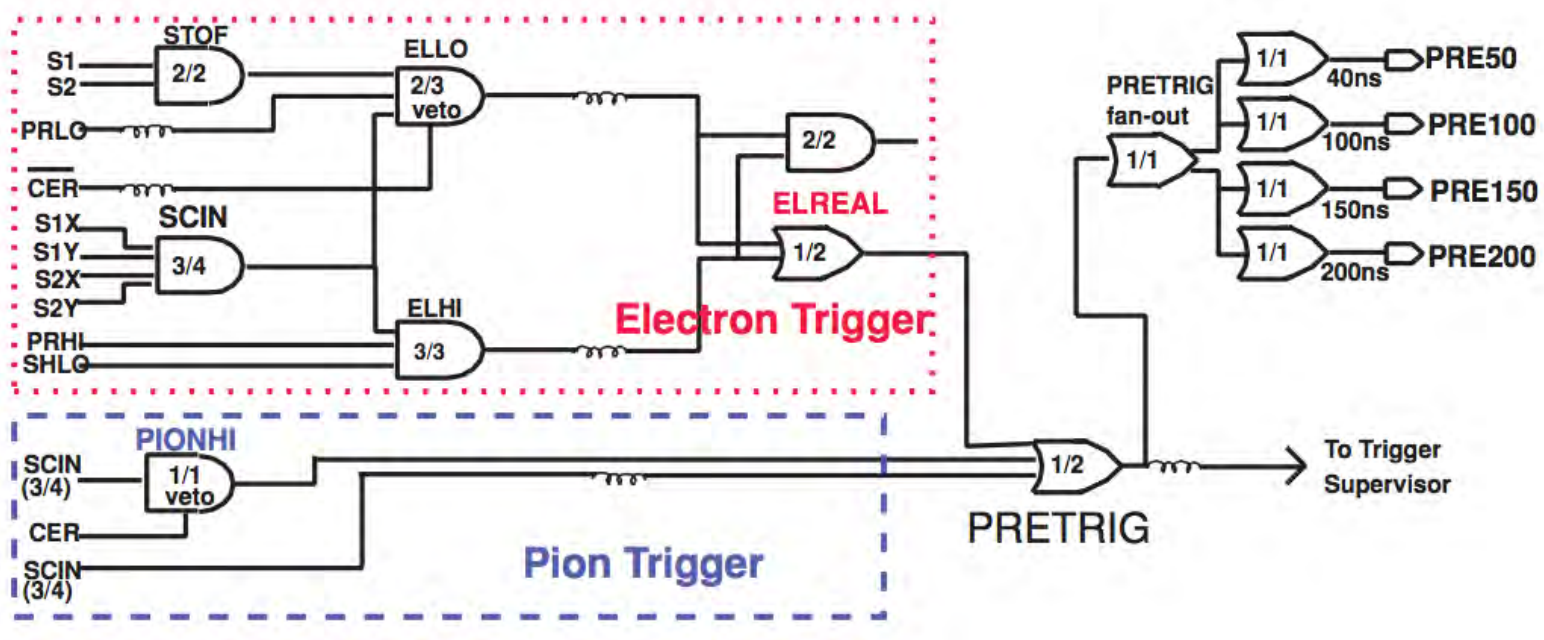

Figure 2.6: Schematic of the spectrometer pretrigger logic. The pre-selection of good electron events is done in the upper part of the system whereas the lower part of the diagram represents the logic used for the selection of good pion events. More details in Ref. [11, 3]. (Original diagram on the way) 
A schematic of the trigger system is shown in Figure 2.6. The SCIN trigger from the hodoscopes is the most basic trigger and it requires a hit in three out of the four scintillator planes. The scintillator information is also used to form the standard electron trigger, ELREAL, which is provided by two signals: the SCIN and the STOF that requires the logical AND of $\mathbf{S 1}$ and $\mathbf{S 2}$. These two signals are then used to give two conditions: ELHI and ELLO (see Figure 2.6). In the hadron-arm, good pion events were acquired using SCIN trigger with an additional requirement of no signal above a given threshold in the Čerenkov detector (PIONHI). Each trigger signal is sent to TDC and read out by the DAQ, which allows for determining the efficiency for a given trigger type. Further discussion on the trigger system and trigger efficiency can be found in Refs. $[2,3,10]$.

The data acquisition system used in Hall $\mathrm{C}$ is known as CODA (CEBAF online data acquisition) version 1.4, which records three types of data for each experimental run. TDC and ADC information for various detectors were recorded by the DAQ on an event-by-event basis, and scalers, for example, the charge and EPICS data from slow controls were read out every 2 and $30 \mathrm{~s}$, respectively. These data were read out over a network through Fastbus and VME crates with readout controlled CPU for each event in the data stream. A detailed discussion on CODA can be found in Ref. [31].

\subsection{Data Analysis}

The raw data collected by the data acquisition system were processed by the standard Hall C analysis software (ENGINE), which decodes the raw data into physical quantities on an event-by-event basis in order to perform the necessary data analysis. Some of the major components of the analysis, in particular, tracking, event reconstruction and optical calibration, are discussed extensively in Refs. [3, 11]. This section focuses on the determination of the normalized experimental yields, as a function of the relevant kinematic quantities, 
including identification of good events, spectrometer acceptance reconstruction, and various correction factors.

\subsubsection{Particle Identification (PID) and Event Selection}

The main particles of interest for the analysis of $\pi^{+}$electroproduction data at high $-t$ are scattered electrons and positively charged pions. Thus, the identification of true $p\left(e, e^{\prime} \pi^{+}\right) n$ events relies heavily on the correct identification of electrons and pions in the respective spectrometers as well as on precise coincidence timing information to separate out "real" from "random" coincidence events.

In the SOS, electrons are detected using a combination of the SOS gas Cerenkov and calorimeter. The gas C̆erenkov, filled with Freon-12 at 1 atm pressure, was used as a threshold detector with a mean SOS signal of seven photoelectrons per electron and a detector efficiency of $99.92 \pm 0.02 \%$ [13]. The criterion used to select good electron events was more than 0.5 photoelectrons $\left(N_{p e}>0.5\right)$ in the C̆erenkov detector. The cut was selected based on the position dependence of the mean photoelectron yield and to ensure good efficiency across the acceptance. Due to the relatively low photoelectron cut used for good electron event selection, some negatively charged pions $\pi^{-}$are able to pass the particle identification requirements. The contamination of pions that were not rejected by the electron trigger and that possess the correct coincidence timing and reconstructed missing mass was less than 2.5\%. In order to suppress these contaminated pions, an additional criterion of a "showersum" greater than $0.7\left(E_{\text {cal }} / p>0.7\right)$ was placed on the SOS calorimeter. The "showersum" is the amount of energy deposited in the calorimeter normalized to the central SOS momentum. The loss of electrons due to this requirement on the SOS calorimeter is less than $0.1 \%$, while the rejection of $\pi^{-}$is better than $99 \%$. The detection efficiency of the SOS calorimeter for the analysis is $99.50 \pm 0.10 \%$. The pion rejection by the SOS calorimeter 
resulted in the total pion contamination smaller than $0.03 \%$. Further information on the detector efficiencies are provided in Ref. [3].

a)

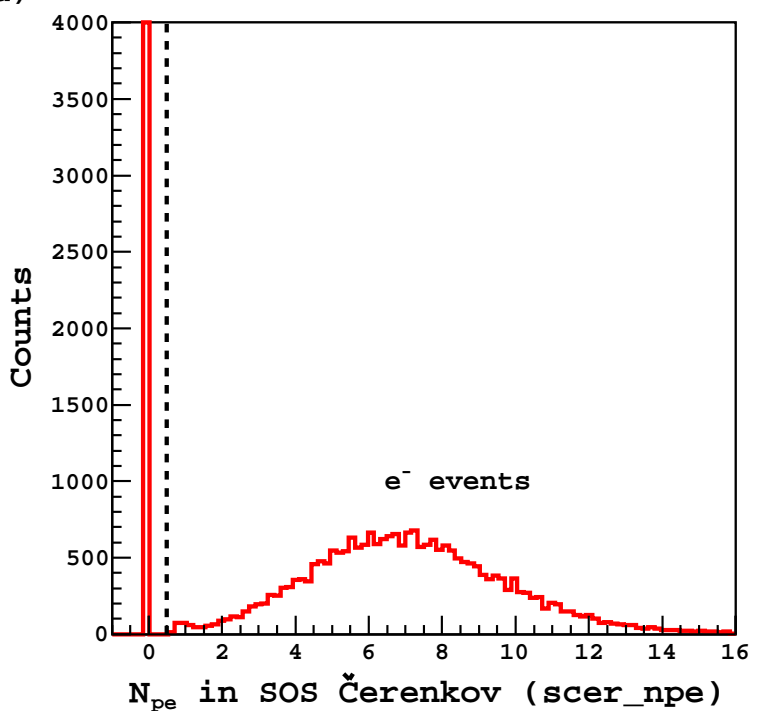

b)

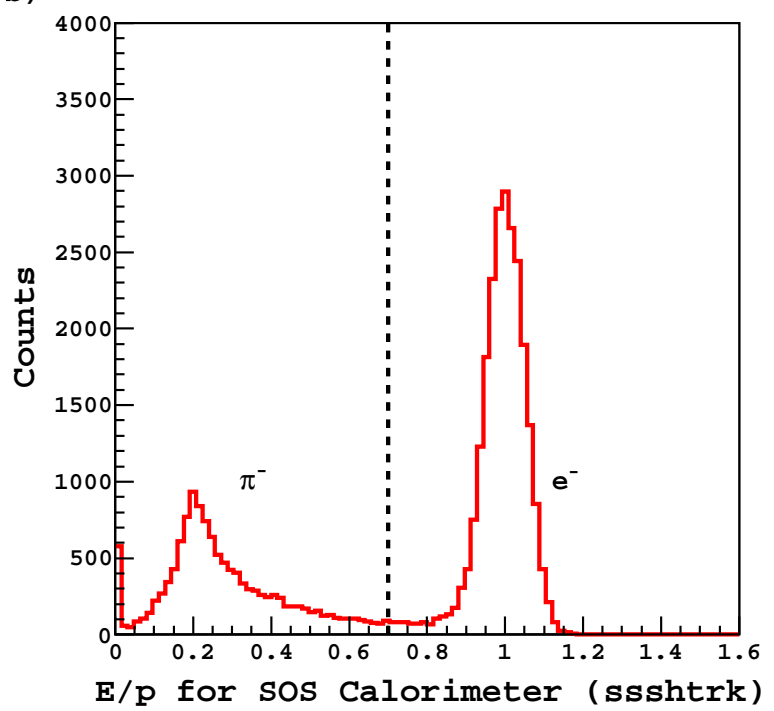

Figure 2.7: The number of photoelectrons in SOS Cerenkov is shown in panel a. The dotted line at 0.5 represents a cut placed to select good electron events. Similarly, the energy deposited in the calorimeter is plotted in panel b. The dotted line at 0.7 represents a cut to separate out $\pi^{-}$and electrons.

In the HMS, where positively charged pions, $\pi^{+}$were detected, the contaminating particles were protons and positrons. Although, in principle, pions do not produce a signal in the HMS gas Čerenkov detector, the probability that pions will produce knock-on electrons while traversing the detector is non-zero, resulting in a number of photoelectrons greater than zero. These pions may then be rejected by the same cut used to eliminate electrons (positrons). In order to account for this, the pion efficiency of the HMS gas Cerenkov was determined with a tight cut on the missing neutron mass and the calorimeter to eliminate electrons. The pion rejection correction used was $99.60 \pm 0.05 \%$ for a cut of $N_{p e}<2.0$. The contaminated positrons that passed the HMS gas C̆erenkov cut were removed by the subtraction of random coincidences in the analysis, which is elaborated further in the next section. Therefore, no offline restrictions were placed for eliminating positrons. Similarly, the 
rejection of proton events is typically achieved via the particle velocity, $\beta=v / c$, calculated from the time-of-flight difference between the two hodoscopes in the HMS detector stack. For our $p\left(e, e^{\prime} \pi^{+}\right) n$ kinematics, the pion and proton momenta were high, resulting in $\beta_{\text {ToF }}$ distributions for pions and protons which were not completely separated. Thus, the HMS aerogel C̆erenkov was used to further discriminate proton events, along with a rather loose $\beta_{\text {ToF }}$ cut (see Subsec. 2.2.2) with $0.10<\beta_{T o F}<1.50$. For a threshold cut of $N_{p e}>3.0$ (the mean number of photoelectrons being 12), the aerogel C̆erenkov efficiency was determined to be $99.50 \pm 0.02 \%$.

a)

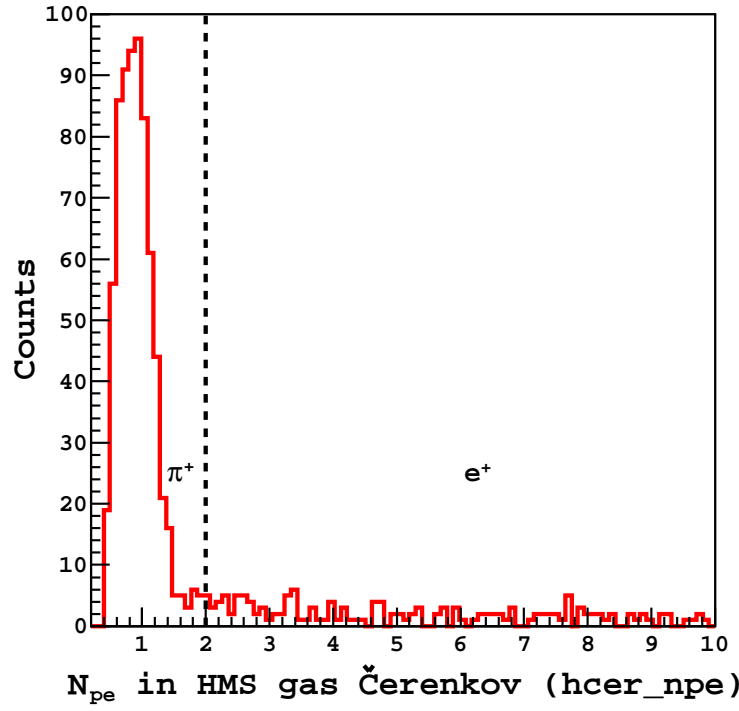

b)

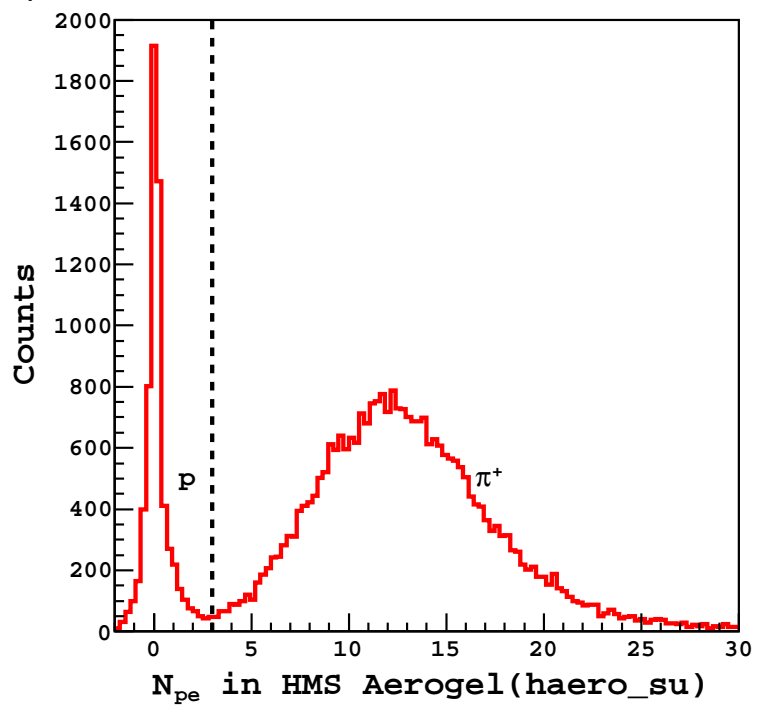

Figure 2.8: The number of photoelectrons in HMS Cerenkov is shown in panel a. The dotted line at 2.0 represents a cut placed to select pions from the positrons. Similarly, the number of photoelectrons in HMS aerogel Cerenkov is plotted in panel b. The dotted line at 3.0 represents a cut to separate out $\pi^{+} /$protons.

\subsubsection{Spectrometer Acceptance Reconstruction}

In addition to selecting particles of interest, the event selection process also entails placing cuts on reconstructed target quantities. The following equation, expressed in terms of power series expansion, is used to perform the reconstruction: 


$$
x_{t a r}^{i}=\sum_{n=j, k, l, m}^{N} M_{j k l m}^{i}\left(x_{f p}\right)^{j}\left(y_{f p}\right)^{k}\left(x_{f p}^{\prime}\right)^{l}\left(y_{f p}^{\prime}\right)^{m}, \quad j+k+l+m \in[0, N]
$$

where $x_{t a r}^{i} \in\left(\delta, y_{t a r}, x_{t a r}^{\prime}, y_{t a r}^{\prime}\right)$ and the $M_{j k l m}^{i}$ represent the reconstruction matrix elements. In particular, the reconstructed quantities, $x_{t a r}^{i}$ in the target system, are the in-plane position $y_{\text {tar }}$ of the event in spectrometer co-ordinate system (refer to Figure 2.9), the in and out of plane scattering angles, $x_{t a r}^{\prime}$ and $y_{t a r}^{\prime}$ respectively, with respect to the spectrometer central angle, and the momentum of the particle, $p$. The latter is commonly expressed as $\delta$, relative to the spectrometer central momentum, $p_{0}$. It is given by

$$
\delta=\frac{\left(p-p_{0}\right)}{p_{0}}
$$

The sum over indices are constrained by $0 \leq j+k+l+m \geq N$, where $N$ is the order of the series expansion. For this analysis, all the matrix elements up to sixth order $(N=6)$ were included for both the HMS and SOS. Further details on the target quantities, event reconstruction, and reconstruction matrix can be found in Refs. [2, 3, 10].

These cuts on reconstructed target quantities are commonly known as spectrometer acceptance cuts. The performances and acceptances of both spectrometers have been extensively studied in prior Hall C experiments $[3,10,13,26]$. Thus, to make sure only the appropriate physics events within the known acceptance of the spectrometers are selected, cuts on reconstructed target variables are necessary. Further information on the acceptances cuts, along with the data and Monte-Carlo comparisons, are provided in Section 2.3.2. A diamond-shaped cut on the $Q^{2}-W$ distribution shown in Figure 2.10 was also placed. Since the kinematic variables are correlated, the average values, $\overline{Q^{2}}$ and $\bar{W}$, can change significantly across various $-t$ settings. In order to keep $\overline{Q^{2}}$ and $\bar{W}$ roughly the same across the $-t$ range, 


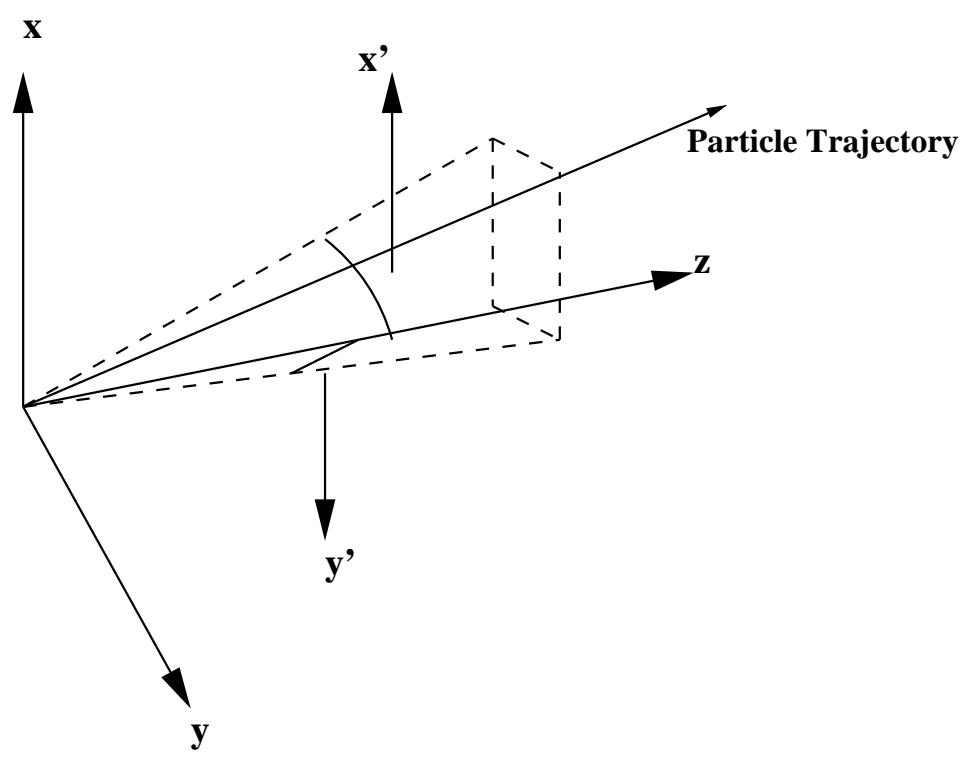

Figure 2.9: Spectrometer co-ordinates system along with the definition of the angles $x^{\prime}$ and $y / . \mathbf{z}$ points along the optical axis at any point inside the spectrometer. $\mathbf{x}$ points outward in the dispersive direction whereas $\mathbf{y}$ completes the right-handed system to the left in the non-dispersive direction [10].

so that - $t$-dependence of the unseparated cross section can be studied, the "diamond" cut was necessary.

\subsubsection{Subtraction of Backgrounds}

The $p\left(e, e^{\prime} \pi^{+}\right) n$ data contains mainly two types of non-physics background events: random coincidence events due to unrelated electrons, pions and/or protons; and coincident electrons and pions originating from the aluminum walls of the target cells. Both of these backgrounds have to be taken into account in calculating the normalized experimental yield. The handling of these background events are further detailed below.

\section{Random Coincidence}

Once the appropriate particle type has been selected and cuts on reconstructed spectrometer quantities (acceptance cuts) have rejected bad events (see Figure 2.13), the random $e-\pi$ coincidence events, constituting a background, have to be eliminated from the data sample. 
The acceptance cuts are further discussed in Sect. 2.3.2. In order to select "real" and "random" $e-\pi$ coincidence events, the distribution of the relative particle velocity ratio, $\beta_{T o F}$, and the so-called HMS coincidence time, cointime, were examined. $\beta_{\text {ToF }}$ is calculated once the particle velocity is determined using the time of flight information provided by the four layers of scintillating hodoscopes in the detector stack, while the raw HMS coincidence time is the relative timing between the HMS and the SOS, in which the start is given by an HMS pretrigger and the stop is given by a delayed coincidence trigger timed by the SOS. The raw coincidence time is corrected for the time difference due to variation in the particle trajectory with respect to the central ray of the spectrometer and the velocity of the particle. The corrected HMS coincidence time allows for a resolution of 200 ps, which is ample to resolve the 2 ns beam structure of the accelerator. A sample distribution of $\beta_{\text {ToF }}$ versus cointime for $Q^{2}=2.50 \mathrm{GeV}^{2}, W=2.00 \mathrm{GeV}$, and $-t=0.272 \mathrm{GeV}^{2}$ is shown in Figure 2.11.

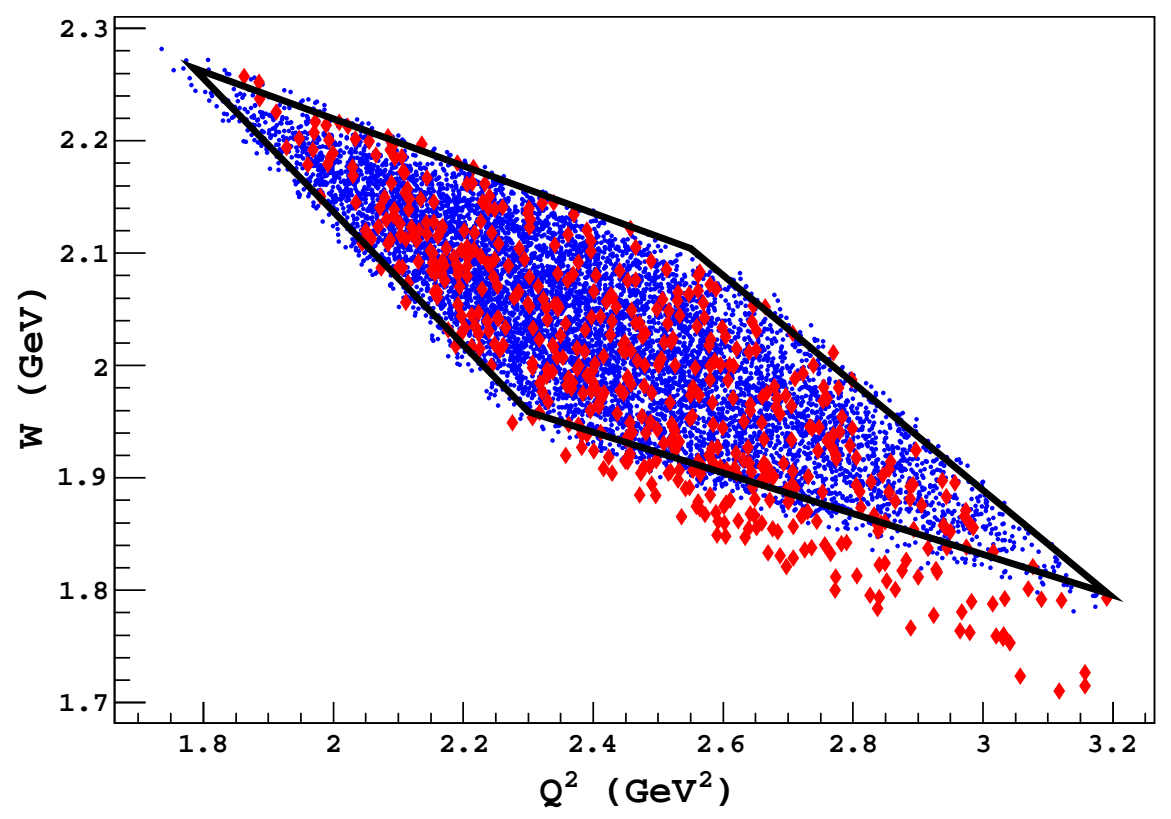

Figure 2.10: $Q^{2}-W$ coverage at two different $-t$ settings. The blue data points represent $-t=0.272 \mathrm{GeV}^{2}$ setting, which was used to define the boundary of the "diamond" shown with solid black line. The red data points show the coverage for $-t=2.127 \mathrm{GeV}^{2}$. (Color Online) 
The random subtraction method used for this analysis is as follows. A cut is placed on the "real" $e-\pi$ coincidence peak of \pm 1 ns, and a sample of seven clean "random" coincidence peaks (three early and four late random coincidences) was taken. Then, the corresponding real and random counts are accumulated. The random counts are then normalized by the number of peaks sampled and subtracted from the "real" peak counts to achieve the total

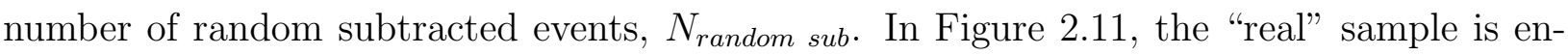
closed by solid red lines, and the "random" sample by dotted blue lines. Both the "real" and "random" coincidence samples have a "blob" and a "tail", as well as some "zero" events. The coincidences of interest, with valid tracking information, is given by the "blob" corresponding

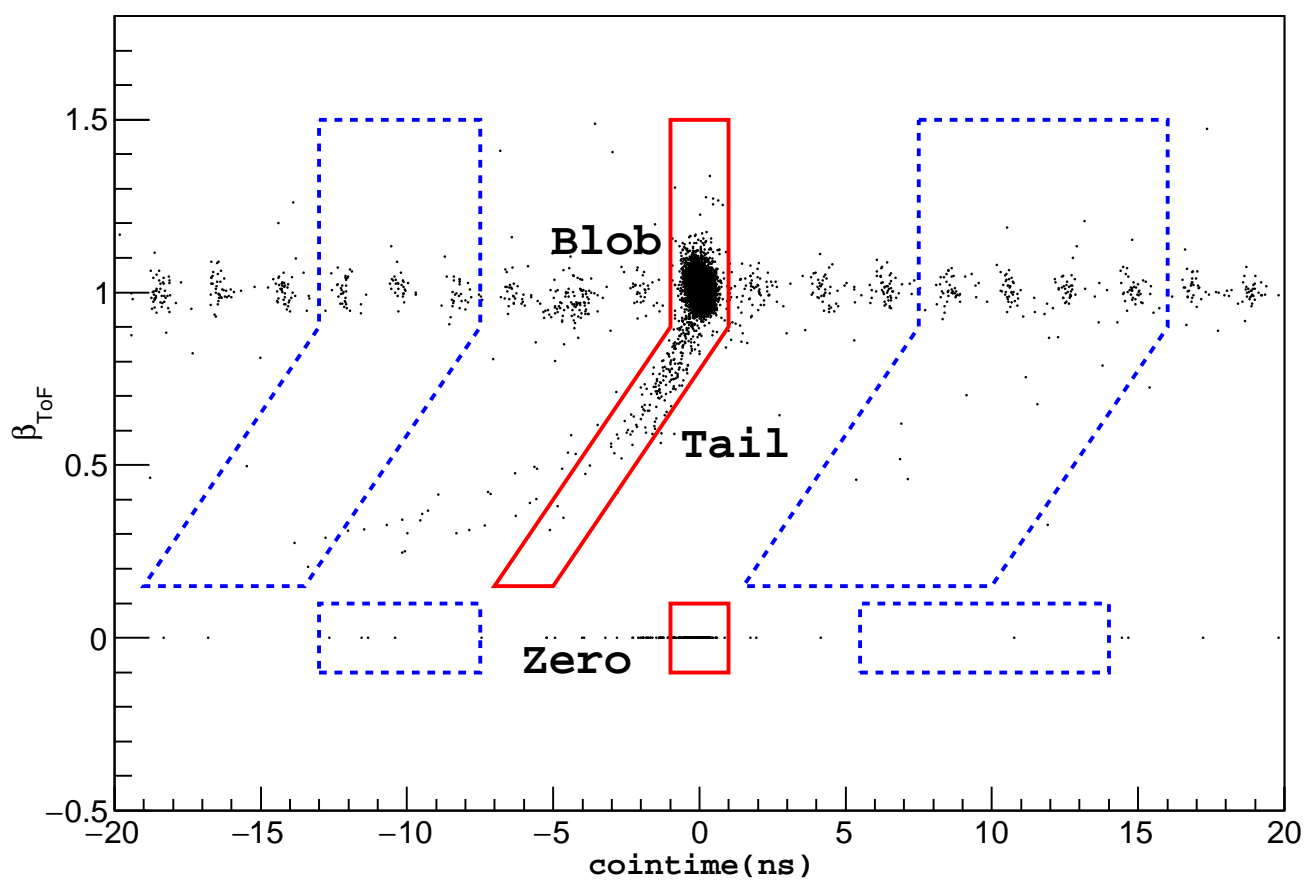

Figure 2.11: $\beta_{\text {ToF }}$ versus cointime distribution after the acceptance and PID cuts for kinematic setting: $Q^{2}=2.50 \mathrm{GeV}^{2}, W=2.00 \mathrm{GeV}$, and $-t=0.272 \mathrm{GeV}^{2}$. Red solid boxes represent the cut used for "real" coincidences, whereas blue dotted boxes show "random" coincidences. The boundaries of the boxes are the same across all the kinematic settings. The blob, tail, and zero, discussed further in the text, are clearly indicated in the figure. (Color Online) 
to $\beta_{T o F}>0.90$. Note that there is a "tail" in the distribution (towards low $\beta_{T o F}$ ) attached to the "blob". This feature, with $\beta_{T o F}<0.90$, is seen because the pions hadronically interact in the scintillator paddles, possibly knocking out a slower hadron, leading to a larger time of flight measurement. These pions pass the drift chambers before interacting in the hodoscope scintillators, the tracking information is likely still valid. Thus, a two-dimensional cut including both "blob" and "tail", as shown in Figure 2.11, was implemented. Furthermore, there are also "zero" events in the $\beta_{\text {ToF }}$ versus cointime distribution. These are the events for which the analyzer fails to find any $\beta_{T o F}$ at all and thus assigns a value of $\beta_{\text {ToF }}=0$ for these events. These events can arise from pions undergoing nuclear interactions and multiple scattering in the hodoscopes, ending in failure of the fiducial cut in the reconstruction algorithm; this in turn will result in a time of flight overflow $\left(\beta_{T o F}=0\right)$. However, like the "tail" events, "zero" events reconstruct to good values of missing mass. Hence, "zero" events combined with the "blob" and "tail" events provide a total $e-\pi$ coincidence events, where events corresponding to cointime $=0$ are the "real" coincidences and the rest after normalization makes up the "random" coincidences.

\section{Target Can Contribution}

Another source of background that has to be removed while accumulating a sample of "good" events is the background due to scattering from the aluminum target cell walls enclosing the cryogen. In order to characterize the effects due to the end caps of the target can, the so-called "dummy" target consisting of two aluminum foils placed at $z= \pm 2$ $\mathrm{cm}$ is used. As explained in Subsec. 2.1.3, the "dummy" target thickness is greater than the nominal $\mathrm{LH}_{2}$ target cell by a factor of 7.773 , resulting in higher luminosity and rapid accumulation of the required background data sample. The contribution of the target cell wall to the total normalized experimental yield is relatively small, $1-3 \%$. 


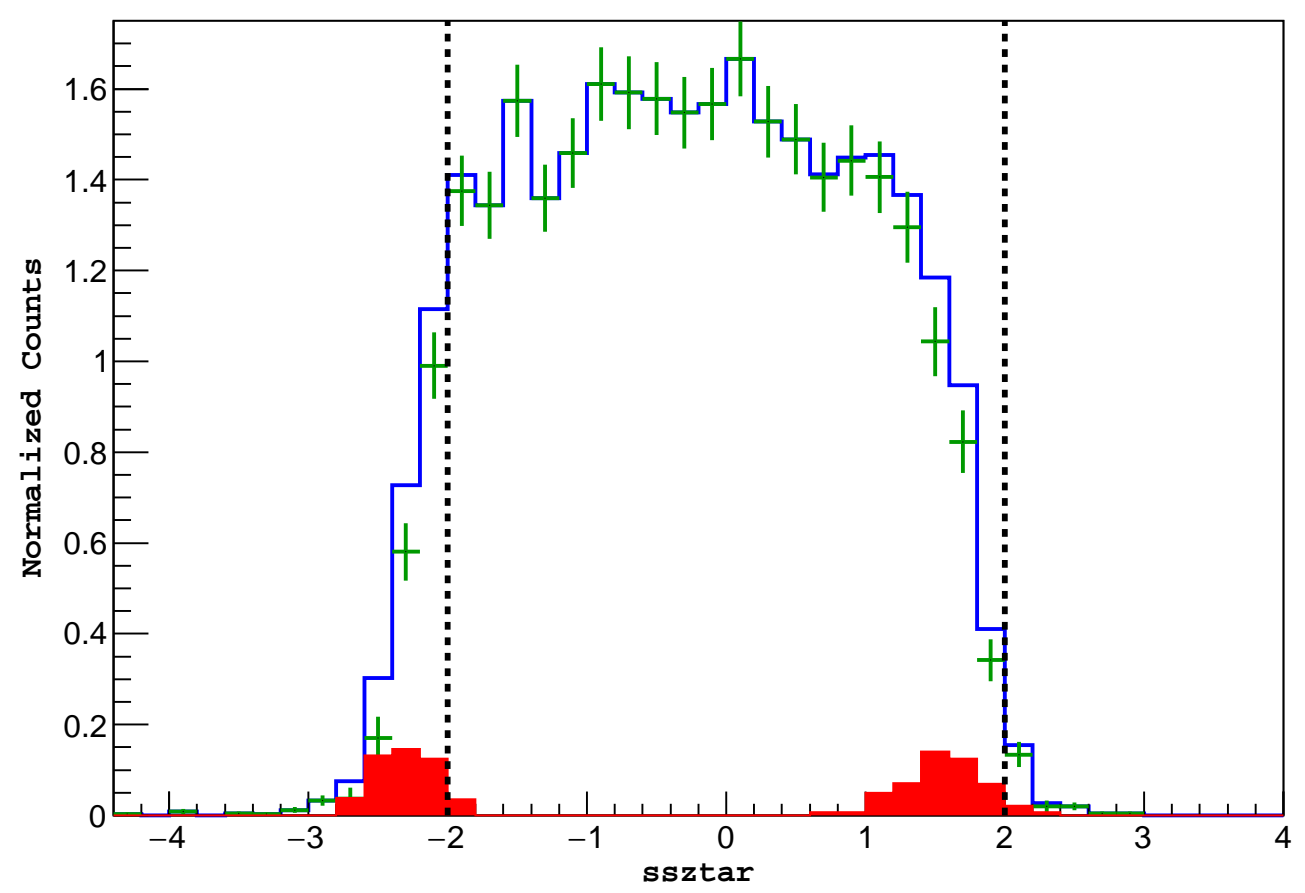

Figure 2.12: Distribution of event vertex position along the beam, ssztar (calculated from SOS $\left.y_{\text {tar }}\right)$ for kinematic setting: $Q^{2}=2.50 \mathrm{GeV}^{2}, W=2.00 \mathrm{GeV}$, and $-t=0.272 \mathrm{GeV}^{2}$. ssztar distribution for events from the $\mathrm{LH}_{2}$ are represented with blue histogram in the figure, while the solid red histogram contain properly weighted events from the dummy target $(\sim 1 \%$ of all events). The green histogram is the dummy subtracted ssztar distribution. Note that the distance between two red bumps is $\sim 4 \mathrm{~cm}$, as expected.

The data for the dummy runs are analyzed in the same manner as the data from the $\mathrm{LH}_{2}$ targets. Figure 2.12 shows the contribution of events from the cell walls. Identical cuts are used and random coincidences are subtracted in a similar fashion for both data samples. Finally, efficiency corrected and charge normalized experimental yields due to the "dummy" target is subtracted from the real cryotarget data, taking into account the thickness difference between the cryotarget cell wall and the "dummy" target. Compared to other experimental uncertainties, the uncertainty stemming from the thickness mismatch between the real and dummy targets is negligible. 


\begin{tabular}{|c|c|c|}
\hline Variable & Value & Reference \\
\hline \multicolumn{3}{|c|}{ PID and Event Selection cuts } \\
\hline$\pi^{+}$in HMS & haero_su $>3.0 \& \&$ hcer_npe $<2.0$ & Subsection 2.2.1 \\
\hline electron in SOS & scer_npe $>0.5 \& \&$ sshtrk $>0.70$ & \\
\hline Coincidence timing and HMS $\beta$ & - & Subsection 2.2.2 \\
\hline \multicolumn{3}{|c|}{ Spectrometers Acceptance Cuts } \\
\hline HMS $|\delta|$ & $\mid$ hsdelta $\mid<8.0$ & Subsection 2.3.2 \\
\hline HMS $\left|y_{t a r}\right|$ & $\mid$ hsytar $\mid<1.75$ & $"$ \\
\hline HMS $\left|x_{t a r}^{\prime}\right|$ & $\mid$ hsxptar $\mid<0.080$ & $"$ \\
\hline HMS $\left|y_{\text {tar }}^{\prime}\right|$ & $\mid$ hsyptar $\mid<0.035$ & $"$ \\
\hline $\operatorname{SOS}|\delta|$ & $\mid$ ssdelta $\mid<15.0$ & $"$ \\
\hline $\operatorname{SOS}\left|y_{\text {tar }}\right|$ & ssytar $<1.50$ & Ref. [3] \\
\hline $\operatorname{SOS}\left|x_{t a r}^{\prime}\right|$ & $\mid$ ssxptar $\mid<0.040$ & Subsection 2.3 .2 \\
\hline SOS $\left|y_{\text {tar }}^{\prime}\right|$ & $\mid$ ssyptar $\mid<0.065$ & Subsection 2.3.2 \\
\hline $\operatorname{SOS}\left|x_{f p}\right|$ & $|s s x f p|<20.0$ & Ref. $[3,10]$ \\
\hline \multicolumn{3}{|c|}{ Kinematic Cuts } \\
\hline Missing Mass (GeV) & $0.92<M_{x}<1.00$ & Figure 2.15 \\
\hline$Q^{2}-W$ cut & - & Figure 2.10 \\
\hline
\end{tabular}

Table 2.3: Overview of the analysis cuts used to select data for the extraction of exclusive pion electroproduction cross sections.

\subsubsection{Analysis Cuts}

An overview of the cuts used in the analysis of these pion electroproduction data at high $-t$ is shown in Table 2.3. The first panel contains coincidence timing and particle identification cuts applied to select the particles of interest, namely, pion and electron as well as "good" coincidence events. The second panel contains cuts on the reconstructed target quantities (see Subsec. 2.3.2) for both HMS and SOS. Outside the limits of these acceptance parameters, the quality of the reconstruction worsens. The last panel in Table 2.4 contains cuts on physics quantities such as $Q^{2}, W$, and the missing mass. The missing mass cut was varied by $\pm 20 \mathrm{MeV}$ in order to estimate the systemtic uncertainty, which is elaborated further in Sec. 2.4.3. 


\subsubsection{Efficiencies and Other Corrections}

After event selection and subtraction of various backgrounds, one must apply corrections for any inefficiencies in which good events may be lost. Some of these, in particular Čerenkov efficiencies, are briefly examined in Subsec. 2.2.1. Other corrections include inefficiencies resulting from track reconstruction, data acquisition dead time, trigger, and absorption of pions in the spectrometers. These are discussed in more detail in Refs. [3, 11, 13]. Here, a brief discussion of few of the important efficiencies are given, along with a table listing all the efficiencies used for the analysis.

The basis of kinematic reconstruction is to find a valid track in the pair of wire chambers in each spectrometer. Each chamber has six planes of wires, and signal in at least five planes is required by the tracking algorithm to start constructing a track for a given event. Fitting a straight line through both the wire chambers, a $\chi^{2}$ minimization is performed by the tracking algorithm. In case the fit results in more than one possible track, the track that comes closest to the scintillator paddle in the second hodoscope that fired is selected. A more detailed information of tracking algorithm is given in Ref. [32]. Projecting the fitted track to the nominal focal plane yields the position $\left(x_{f p}, y_{f p}\right)$ and Euler angles $\left(x_{f p}^{\prime}, y_{f p}^{\prime}\right)$ of the particle at the focal plane.

The tracking efficiency is defined as the probability that the tracking algorithm found a valid track for a particle identified as an electron (or pion). It depends both on the efficiency of the drift chambers and on the tracking algorithm. The PID requirements eliminate the bias introduced by the presence of other particle types. The HMS tracking efficiency was $97 \%$, where as the SOS tracking efficiency was slightly worse at $96 \%$.

At high rates, the probability of more than one particle passing through the drift chamber within the 200 ns TDC window used in this analysis is non-zero. The tracking algorithm only determines one "best" track for each event; additional tracks are taken into account 
with the electronic or computer dead-time corrections. However, it has been observed in Ref. [11] that the efficiency for finding a single track in the presence of multiple tracks is significantly lower, primarily due to limitation of software in handling the multiple hits. The rate dependence of the tracking efficiency then mostly comes about from the increased probability of having multiple tracks at high rates. A rate-dependent tracking efficiency calculation, detailed in Ref. [3], was developed to resolve the issue.

Additionally, a fraction of the produced pions are lost as a result of nuclear interactions in the materials while traversing the HMS before reaching the detectors in the detector hut. The loss of pions is mainly because of absorption and large angle scattering. The transmission of pions through the spectrometer was calculated using the list of traverse materials and the pion-nucleon reaction cross section, which includes absorption and inelastic reactions. The calculation was performed for the $\mathrm{F}_{\pi}-2$ experiment and the details are provided in Ref. $[11,3]$.

\begin{tabular}{|c|c|c|}
\hline \multicolumn{3}{|c|}{ Summary of Correction Factors } \\
\hline HMS Tracking efficiency correction & 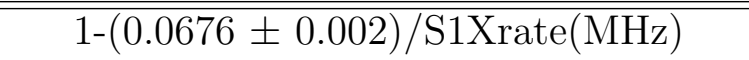 & Ref. [3] \\
\hline$\beta$-cut correction & $2.42 \pm 0.12 \%$ & Ref. $[3,11]$ \\
\hline Pion Absorption & $2.0 \pm 1.0 \%$ & Subsec. 2.2 .5 \\
\hline SOS Čerenkov efficiency & $99.92 \pm 0.02 \%$ & Refs. $[3,11]$ \\
\hline SOS Calorimeter efficiency & $99.50 \pm 0.10 \%$ & n \\
\hline HMS C̆erenkov efficiency & $99.60 \pm 0.05 \%$ & $"$ \\
\hline HMS Aerogel efficiency & $99.50 \pm 0.02 \%$ & $"$ \\
\hline Coincidence time blocking & $e^{- \text {SOSPretrigrate } *(92 n s)}$ & $"$ \\
\hline HMS electronic live time & 1 - 6/5(hPRE100 - hPRE150)/hPRE100 & Refs. [3] \\
\hline SOS electronic live time & 1 - 6/5(sPRE100 - sPRE150)/sPRE100 & \\
\hline
\end{tabular}

Table 2.4: Summary of correction factors applied to the experimental data. Here, S1Xrate represents the rate in the first scintillator layer, which is used to determine the HMS tracking efficiency. Similarly, the pre-trigger(PREXXX) signals from the scalers at varying limiting gate widths are used to determine the electronic live times corrections for both spectrometers. In particular, PRE100 and PRE150 denote the total number of counts with corresponding gate widths of $100 \mathrm{~ns}$ and $150 \mathrm{~ns}$ respectively [3].

Once all of the corrections listed in Table 2.4 are applied to the experimental data, one 
can proceed further to determine the total experimental yield, which is given by the following equation.

$$
Y_{\text {exp }}=\frac{N_{\text {random sub }}}{\varepsilon_{\text {total }} \cdot Q_{\text {total }}}
$$

Here, $N_{\text {random sub }}$ is the total number of random subtracted coincidence events (i.e. $N_{\text {random sub }}=N_{\text {real }}-N_{\text {random }}$ ), $\varepsilon_{\text {total }}$ is the efficiency correction factor (all efficiencies combined) and $Q_{\text {total }}$ is the total accumulated beam charge in $\mathrm{mC}$.

\subsection{SIMC}

The standard Hall C Monte Carlo package (SIMC), which was used for the analysis of several previous Hall $\mathrm{C}$ experiments, has been described in detail elsewhere (Refs. $[2,3,13])$. Therefore, this section will provide a brief overview of the program. In addition to the overview, comparison between the data and simulation will also be done for various optics as well as physics variables in this section.

\subsubsection{Overview}

The primary framework for SIMC is based on the code SIMULATE, which was designed for SLAC experiment NE18 [33]. While the general structure of the program has been kept similar, the code had to be modified to include the Hall $\mathrm{C}$ co-ordinate system and Monte Carlo simulations of the Hall $\mathrm{C}$ spectrometers combination, with the main purpose of characterizing the limited acceptances of both magnetic spectrometers. The Hall C coordinate system, which is different than the spectrometer co-ordinates, is defined as follows $[10]:$

* $\mathbf{x}$ points to the right of the beam (downstream), in the horizontal plane, 
* $\mathbf{y}$ points down towards the floor,

* $\mathbf{z}$ points along the direction of beam (downstream).

SIMC was designed as an aperture checking Monte Carlo and hence does not have the capability to simulate individual detector signals. However, SIMC includes all relevant details of the experimental conditions such as the rastering and energy spread of the electron beam, radiative processes, hadron decay, multiple scattering, ionization energy loss and finite track resolution [13] to ensure a realistic simulation of the experimental data. SIMC allows both polarized and unpolarized target configurations.

Both the initial co-ordinates of the interaction vertex $(x, y, z)$, and the kinematic properties such as direction and momentum for the particles of interest, are generated randomly by the Monte Carlo for each event. The starting values for the generation limits in angle and momentum are fixed by the input to the simulation, which are typically chosen to exceed the physical acceptance of the spectrometers. The outgoing particles are radiated and followed on the trip through the target, taking into account energy loss and multiple scattering, only if the kinematics of that particular event are physically allowed. The spectrometer co-ordinate system, shown in Figure 2.9, which is different than the Hall C co-ordinate system described earlier, is used to generate all of the angular information at the vertex. Once the event generation process is complete in SIMC, the events are sent to the single-arm spectrometer modules, which simulate the magnetic optics inside the Hall $\mathrm{C}$ spectrometers and propagate the particle forward through apertures and magnets into the relevant detector hut using a COSY INFINITY [34] magnetic transport model of the respective spectrometer. The COSY model consists of matrix elements which transport the particle sequentially through the magnetic optics in the spectrometer. Inside the detector hut, the trajectory of the particle of interest is tested at each detector aperture. All the events that clear all the apertures and cross the minimum number of detectors in the huts are considered to produce a valid 
trigger. Once a valid trigger is generated, the particle trajectories are back-reconstructed to the target. The event simulation does not take into account the inefficiencies, since only detector apertures are simulated.

Each reconstructed event is weighted by the relevant model cross section corrected for radiative processes, and the overall luminosity factor. The model cross section for a simulated event is expressed in terms of the center-of-mass (CM) photoproduction cross section, $\frac{d^{2} \sigma}{d \Omega_{K}^{*}}$, and virtual photon flux factor, $\Gamma_{\nu}$. It is given by five-fold differential form of Equation 1.3:

$$
\frac{d^{5} \sigma}{d E^{\prime} d \Omega_{e^{\prime}} d \Omega_{\pi}^{*}}=\Gamma_{\nu} \frac{d^{2} \sigma}{d \Omega_{\pi}^{*}}
$$

However, the relevant physics quantities such as the interaction "vertex", the electron and pion directions, and the electron momentum (the hadron momentum is determined by energy and momentum conservation) are generated in the laboratory frame. Thus, a transformation has to be made in order to express the model cross section as the Lorentz invariant quantity [3], which is given by following equation.

$$
\frac{d^{2} \sigma}{d \Omega_{\pi}^{*}}=\left(\frac{d t}{d \cos \theta^{*}}\right) \cdot\left(\frac{d^{2} \sigma}{d t d \phi}\right)
$$

where $d t / d \cos \theta^{*}=2\left|\mathbf{p}_{\pi}^{*}\right|\left|\mathbf{q}^{*}\right|$ is the Jacobian factor to transform from CM to lab frame, and $\left|\mathbf{p}_{\mathbf{K}}^{*}\right|$ and $\left|\mathbf{q}^{*}\right|$ are the three-momenta in the CM frame of the electroproduced pion and the virtual photon.

\subsubsection{Data and Monte Carlo comparison}

The target quantities $\left(\delta, y_{t a r}, x_{t a r}^{\prime}, y_{t a r}^{\prime}\right)$ in SIMC are reconstructed with realistic wire chamber resolutions using a set of reconstruction matrix elements. The simulated reconstructed quantities can be compared to the experimental data as a check on the model cross section as well as the spectrometer optics models. The HMS reconstructed target quantities 
in SIMC are compared with the experimental data in Figure 2.13. If the detector setup is realistically simulated in SIMC, the edges of the measured and simulated target quantities should match. In our analysis, the Monte Carlo simulation generally describes the data quite well, except for the edges of the $y_{\text {tar }}$ distribution in panel b. This effect is well-documented in Refs. [3, 11]; the mismatch is because of $y_{\text {tar }}$ acceptance not being described properly in the SIMC. The quantity does not, however, contribute towards the calculation of the relevant physics variables; only fractional momentum, $\delta$, and spectrometer angles, $x_{t a r}^{\prime}$ and $y_{\text {tar }}^{\prime}$, are used for this purpose. Thus, the quantity, $y_{t a r}$, was not optimized further. The HMS acceptance cuts placed on each of the four target variables are shown with black dotted lines in Figure 2.13. The acceptance limits used in the simulation typically surpass the physical acceptance of the spectrometers. Therefore, to select the "good" events, acceptance cuts (see Table 2.3) for both spectrometers need to be placed on the relevant target variables.

The relevant physics variables are calculated using the reconstructed target variables (except $y_{\text {tar }}$ ). Figure 2.14 shows the comparison of the simulated and experimental distributions of several physics variables. As seen in figure, the Monte Carlo and data agree with each other reasonably well. Since a cross section weight is applied to each event, the agreement of the simulated and experimental distributions of the one dimensional projection of physics quantities such as, $Q^{2}, W$, and $-t$, serves as an additional check on the cross section model used in the SIMC. Moreover, the comparison of the physics quantities also yields information about the description of the kinematic dependence of the cross section.

To account of the so-called radiative effects, i.e., emission of photons by the incoming and/or outgoing electron and/or hadron via the Bremsstrahlung process, a correction factor was applied in SIMC. The radiation correction is implemented in the SIMC by simulating the effects of radiation in the Monte Carlo of the experiment and iterating the input model of the fundamental physics process of interest. For exclusive $\pi^{+}$electroproduction, the radiative 
a)

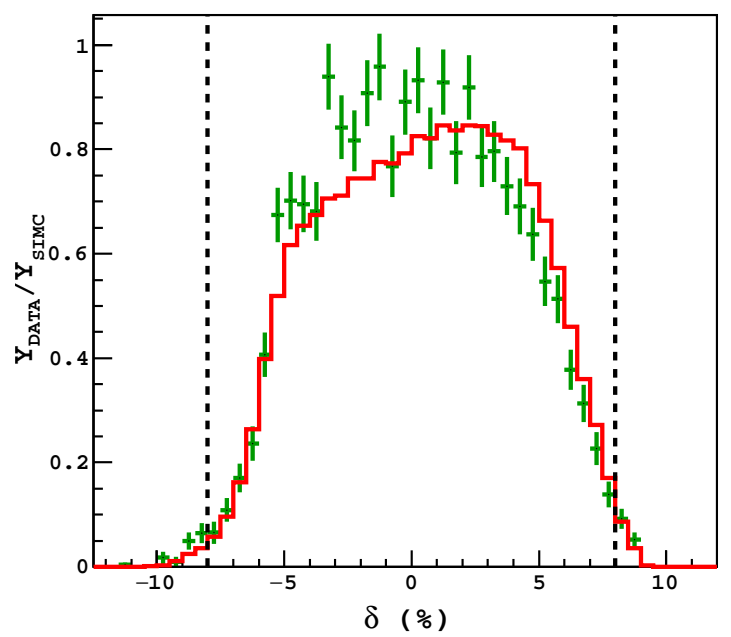

c)

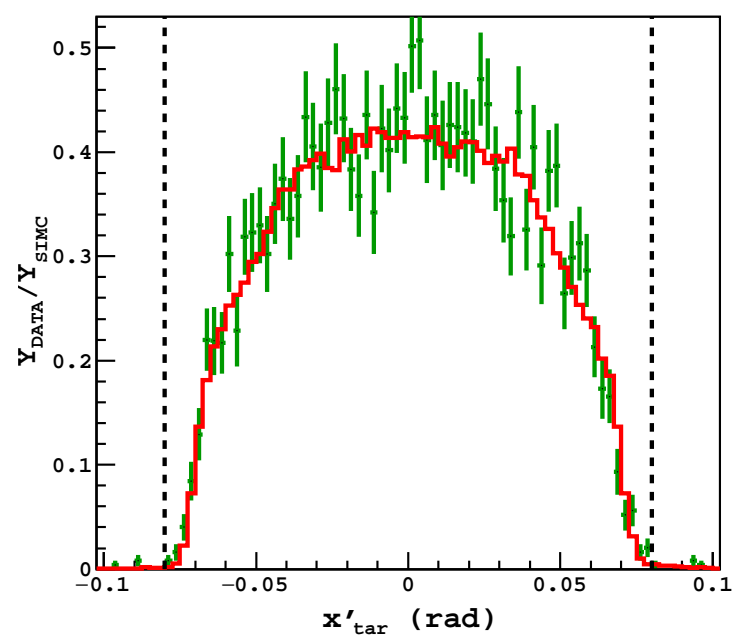

b)

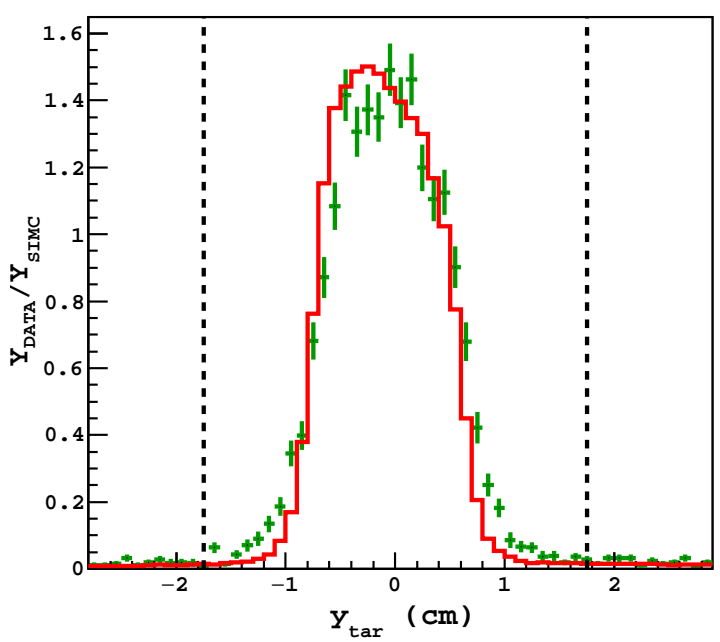

d)

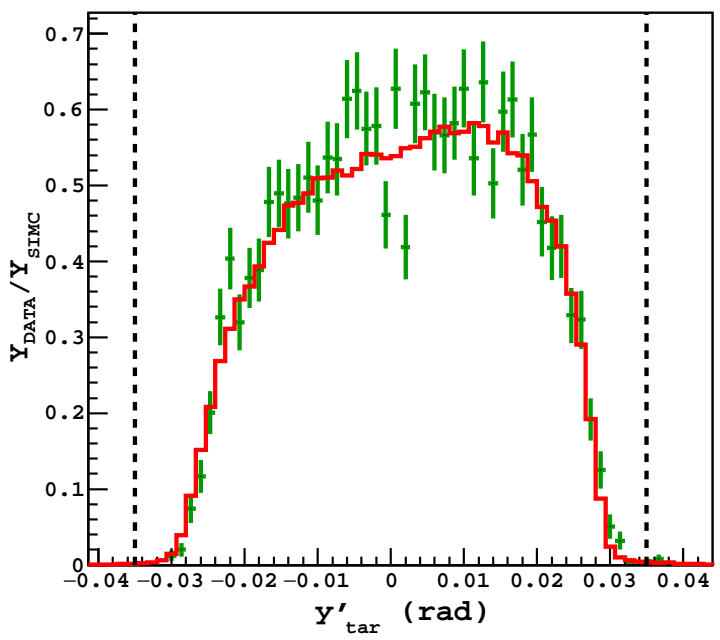

Figure 2.13: Comparison between experimental data (green crosses) and SIMC (red histograms) charge normalized counts for HMS reconstructed target quantities at kinematic setting $Q^{2}=2.50 \mathrm{GeV}^{2}, W=2.00 \mathrm{GeV}$, and $-t=0.378 \mathrm{GeV}^{2}$. The black dotted lines represent the cuts placed on the target quantities, based on the known acceptance of the spectrometer. (Color Online) 
a)

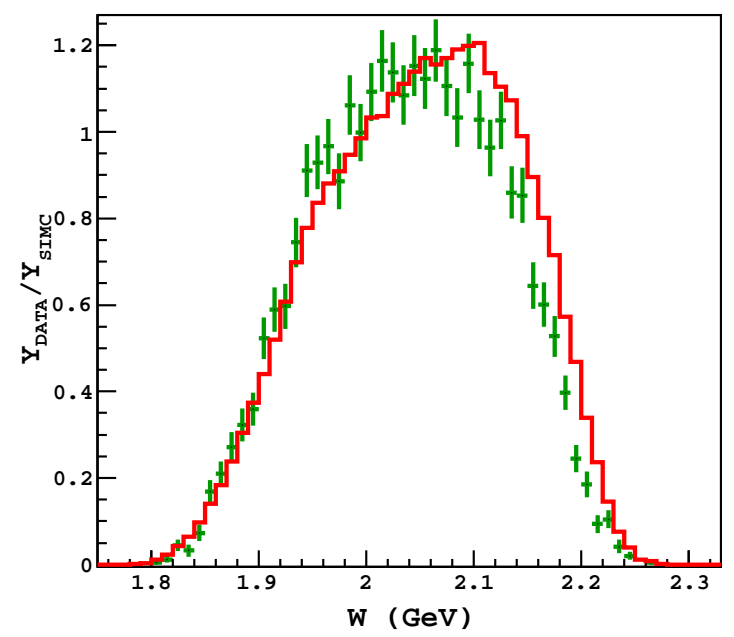

c)

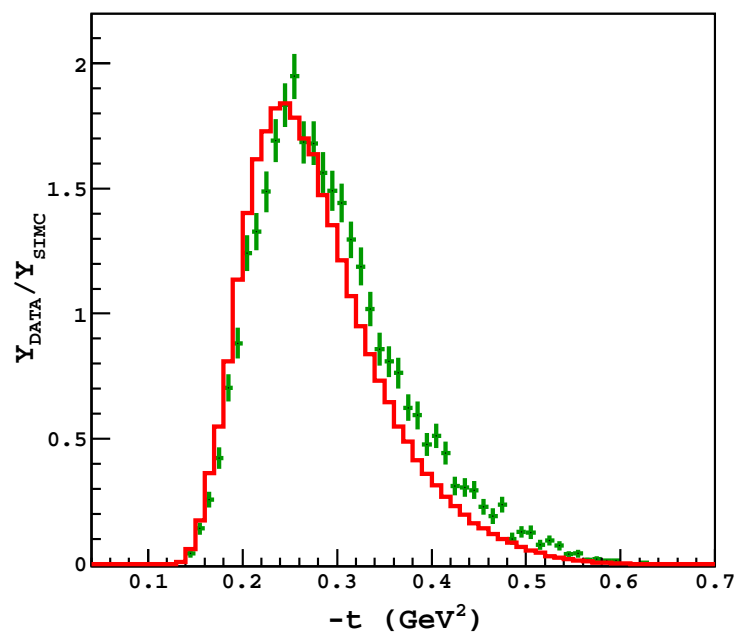

b)

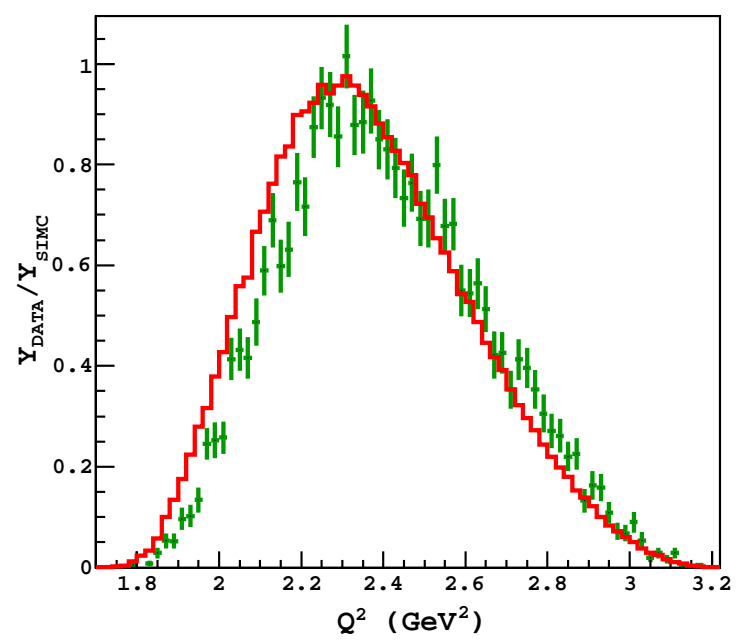

d)

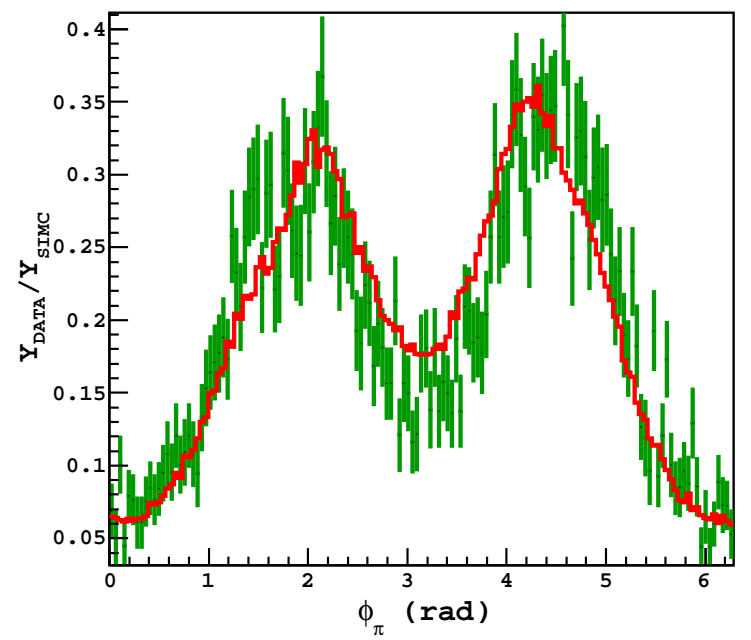

Figure 2.14: Comparison between experimental data (green crosses) and SIMC (red histograms) charge normalized counts for physics quantities at kinematic setting: $Q^{2}=2.50$ $\mathrm{GeV}^{2}, W=2.00 \mathrm{GeV}$, and $-t=0.272 \mathrm{GeV}^{2} . W, Q^{2},-t$, and $\phi_{\pi}$ are shown in panels a, b, c, and d respectively (kinematic variables are defined in Chapter 1). Similar agreements between data and Monte Carlo were observed for all the kinematic settings. (Color Online) 
effects are calculated considering the target is a stationary proton and the pion is taken to be an off-shell proton. The correction does not take into account the contribution from the two-photon exchange diagrams; however, it is expected to be very small. The radiative correction used in SIMC relies on two important approximations. The first of these is the soft photon approximation, where the energy of the radiation photon is restricted to be much smaller than the energies of the initial and final state particles. The second assumption made in SIMC is the extended peaking approximation. In this approximation, the radiation at the one photon level is taken to be in three discrete directions: along the direction of the incoming electron, of the scattered electron, and of the electroproduced pion. The formalism used is discussed more thoroughly in Ref. [35].

In Figure 2.15, the missing mass $\left(M_{x}\right)$ distribution for the data and SIMC is compared. The missing mass is reconstructed using the four-momenta of the scattered electron and the emitted pion and is given by:

$$
M_{x}=\sqrt{\left(\omega-E_{\pi}\right)^{2}-\left(\mathbf{q}-\mathbf{p}_{\pi}\right)^{2}},
$$

where $(\omega, \mathbf{q})$ is the four-momentum of the virtual photon and $\left(E_{\pi}, \mathbf{p}_{\pi}\right)$ is the four-momentum of the emitted pion. One of the ways to check the radiation corrections used in the MonteCarlo is to compare the shape of the radiative tail at higher missing mass region with the data. This shape is largely dominated by radiative effects. In addition, the events that pass through the collimator also contribute to the radiative tail of the missing mass distribution. The collimators used in the experiment are very effective at stopping electrons, but not in the case of hadrons. In particular, some fraction of pions blocked by the HMS collimator are able to pass through, undergoing only multiple scattering and ionization energy loss. The contribution of both the effects described here to the reconstructed missing mass is small $(<5 \%)$. 


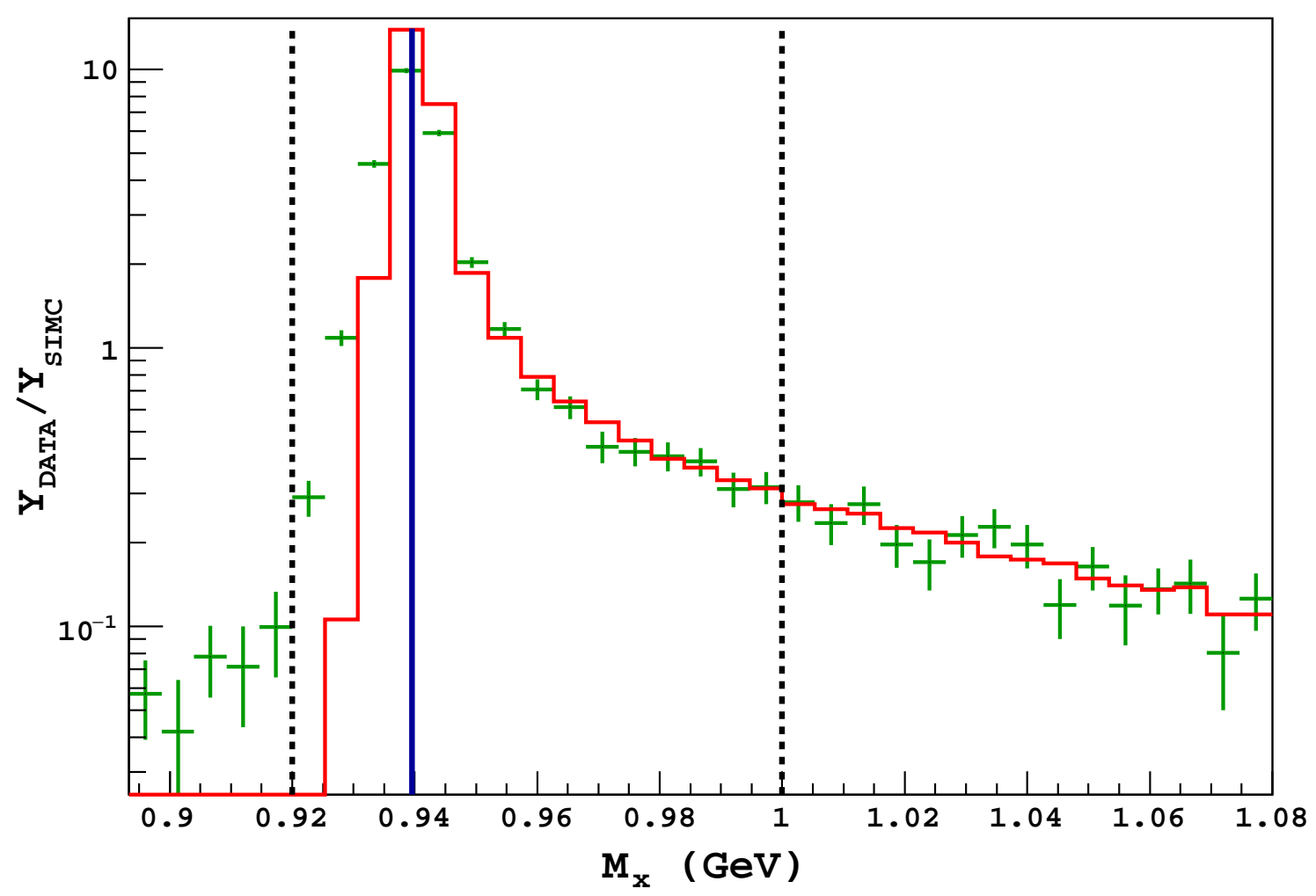

Figure 2.15: Comparison between experimental data (green crosses) and SIMC (red histograms) charge normalized counts for the missing mass $\left(M_{x}\right)$ distribution at $Q^{2}=2.50$ $\mathrm{GeV}^{2}, W=2.00 \mathrm{GeV}$, and $-t=0.272 \mathrm{GeV}^{2}$. With the radiative and collimator corrections, the simulated radiative tails give good description of the measured one. However, there is some disagreement in the lower missing mass region due to multiple scattering inside the target chambers and spectrometers. The solid blue line represent the PDG accepted neutron mass of $0.9396 \mathrm{GeV}$, whereas the two dotted lines at 0.92 and $1.00 \mathrm{GeV}$ represent a cut on the allowed missing mass for the analysis to ensure that no additional pions were produced. The cut will also reduce random coincidence background from events with larger inelasticity than $p\left(e, e^{\prime} \pi^{+}\right) n$ reaction and was varied to study the systematics (see Subsec. 2.4.3). (Color Online)

\subsection{Determination of the cross section}

The Hall C Monte Carlo, SIMC, plays an important part in the determination of the experimental cross section. It is done by comparing the experimental yields to those given by SIMC. As discussed earlier before, the Monte Carlo includes a model of the electroproduction cross section and applies corrections such as spectrometer acceptance, ionization energy loss, 
multiple scattering, radiative corrections, and pion decay. Given that the SIMC simulates these experimental effects appropriately, one can extract the experimental cross section using an iterative process until a good agreement between the data and Monte Carlo is achieved.

\subsubsection{Method}

The determination of the experimental cross section relies heavily on the comparison of the measured experimental yield to the results of a Monte Carlo simulation for the actual experimental set-up, in which a realistic cross section model is implemented. When the model input to the Monte Carlo describes the dependence of the cross section on all kinematic variables $\left(W, Q^{2},-t, \theta_{\pi}\right.$, and $\left.\phi_{\pi}\right)$ correctly (i.e. the ratio of experimental to simulated yield is close to unity within statistical uncertainty), the cross section for any values of $\bar{W}$ and $\overline{Q^{2}}$ within the acceptance can be determined as

$$
\left.\left(\frac{d^{2} \sigma}{d t d \phi}\right)_{\bar{W}, \overline{Q^{2}}}^{\exp }\right|_{\phi_{\pi}=\pi}=\left.\frac{Y_{\exp }}{Y_{\text {sim }}}\left(\frac{d^{2} \sigma}{d t d \phi}\right)_{\bar{W}, \overline{Q^{2}}}^{\text {model }}\right|_{\phi_{\pi}=\pi},
$$

where $Y_{\exp }$ is the charge normalized and efficiency corrected experimental yield integrated over the kinematic acceptance and $Y_{\text {sim }}$ is the equivalent simulated yield resulting from the input model cross section at $\phi_{\pi}=\pi$. The barred quantities in the expression above indicate the acceptance weighted averages for the corresponding quantities. The model cross section provides the appropriate cross section weighting of the kinematic acceptance and also takes care of bin centering corrections to the experimental cross section [3].

After the calculation of "dummy" contribution subtracted, efficiency corrected, charge normalized experimental yield using Equation 2.3, the equivalent simulated yield has to be determined in order to extract the experimental cross section by comparing data and Monte Carlo. The simulated yield has to be normalized to $1 \mathrm{mC}$ of beam charge as the experimental yield is expressed in counts per mC. The Monte Carlo luminosity, $(L)$, is given by, 


$$
L=\frac{\rho t N_{A}}{M}
$$

where $\rho$ is the target density in $\mathrm{g} / \mathrm{cm}^{2}, t$ is the target thickness in $\mathrm{cm}, N_{A}$ is the Avogadro's number and $M$ is the target mass in amu. Using the general case of the simulated yield in SIMC for coincidence experiments described in Ref. [3, 13], the simulated yield can be calculated for pion electroproduction from hydrogen in a relatively easy manner. This is because the pion momentum is constrained by the electron kinematic quantities and the emitted pion direction by the exclusive nature of the reaction. The six-fold model cross section then reduces to five-fold and the simulated yield can be expressed as,

$$
Y_{S I M C}=L \int_{V}\left(\frac{d^{5} \sigma}{d \Omega_{e} d E_{e} d \Omega_{\pi}}\right)^{\text {model }} A(V) R(V) J\left(\Omega \rightarrow \mathbf{X}^{\prime}\right) d \mathbf{X}_{\mathbf{e}}^{\prime} d E_{e} d \mathbf{X}_{\pi}^{\prime}
$$

where $A$ is the coincidence acceptance function, $R$ is the radiative correction factor, $d \mathbf{X}^{\prime}=$ $d x^{\prime} d y^{\prime}$ is the differential solid angle in the spectrometer co-ordinates, and $J$ is the Jacobian for transforming the model cross section from "physics" to the spectrometer coordinates (also used for event generation), given by,

$$
J\left(\Omega \rightarrow \mathbf{X}^{\prime}\right)=\frac{1}{\left(1+\left(y_{e}^{\prime}\right)^{2}+\left(x_{e}^{\prime}\right)^{2}\right)^{3 / 2} \cdot\left(1+\left(y_{\pi}^{\prime}\right)^{2}+\left(x_{\pi}^{\prime}\right)^{2}\right)^{3 / 2}} .
$$

The technique used in this analysis entails evaluating the cross section at a particular point in the experimental acceptance using a model cross section, the so-called "bincentering" method. Since the approach is sensitive to the detailed structure of the model across the experimental acceptance, an iterative procedure is used to optimize the kinematic dependencies of the Monte Carlo to the experimental data. The experimental cross section is extracted using Equation 2.7 and the central model cross section is evaluated using the same model as the one used in the Monte Carlo. 


\subsubsection{Model cross section}

The model cross section for this analysis was determined using an iterative fitting procedure. The starting pion electroproduction cross section model used in the simulation is based on a cross section parameterization developed during the $\mathrm{F}_{\pi^{-}}-2$ analysis, which is given by following equations [11]:

$$
\begin{aligned}
\frac{d \sigma_{L}}{d t} & =\frac{350 Q^{2}}{\left(1+1.77 Q^{2}+0.05 Q^{4}\right)^{2}} \cdot e^{\left(16-7.5 \ln Q^{2}\right) t}, \\
\frac{d \sigma_{T}}{d t} & =\frac{4.5}{Q^{2}}+\frac{2.0}{Q^{4}}, \\
\frac{d \sigma_{L T}}{d t} & =\left(\exp \left[0.79+\frac{2.4}{\sqrt{Q^{2}} t}\right]+1.1+\frac{3.6}{Q^{4}}\right) \sin \left(\theta^{*}\right), \\
\frac{d \sigma_{T T}}{d t} & =-\frac{5.0}{Q^{2}} \frac{|t|}{\left(|t|+m_{\pi}^{2}\right)^{2}} \sin ^{2}\left(\theta^{*}\right) .
\end{aligned}
$$

Here, the cross sections have units of $\mu \mathrm{b} / \mathrm{GeV}^{2}$, the units of $Q^{2}, t$, and $m_{\pi}^{2}$ are $\mathrm{GeV}^{2}$, and $\theta^{*}$ has unit of radians. The initial model input cross section, $\left(\sigma^{\text {model }}\right)$, was then constructed using Rosenbluth formula given in Equation 1.3. Since the $-t$ range of the $\mathrm{F}_{\pi}-2$ kinematics goes to a maximum of only $0.365 \mathrm{GeV}^{2}$, compared to $2.127 \mathrm{GeV}^{2}$ for these data, the parameterization above was not expected to work with the high $-t$ data. The experimental cross section, $\sigma^{e x p}$, along with the fit as a function of $-t$, and yield ratio using the $\mathrm{F}_{\pi}-2$ parameterization as a model input cross section is shown in panels a and b of Figure 2.16, respectively.

The parameterization (see 2.16) of $t$-dependence of the experimental cross sections, extracted using Equation 2.11, was implemented as the input model cross section for the next iteration. This procedure was iterated until the yield ratios were close to unity and $\sigma^{e x p}$ changed by less than a prescribed amount (typically 1\%). The model cross section was taken as the product of global functions describing $W$ and $Q^{2}$ dependences times $t$-dependent parameterized function for the unseparated experimental cross sections. The $W$-dependence 
a)

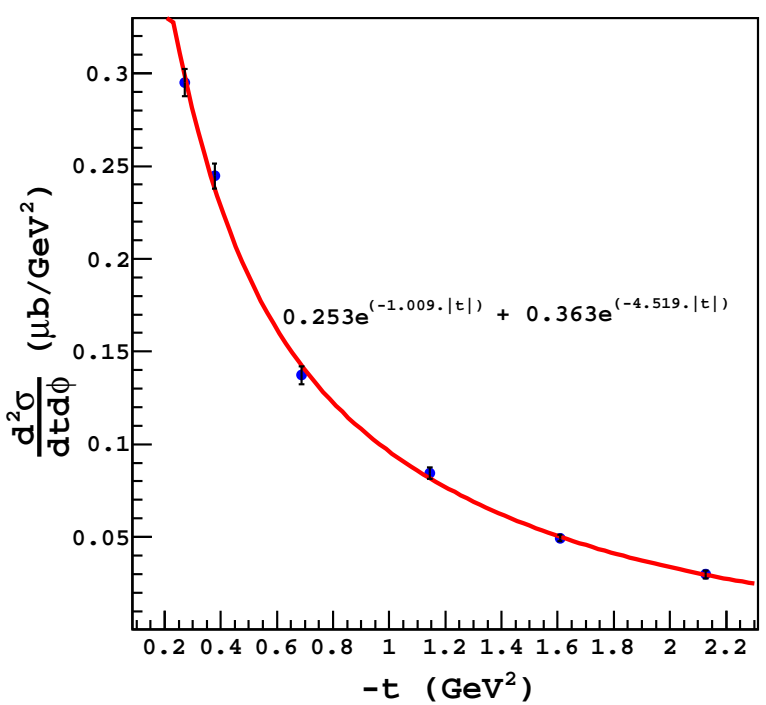

b)

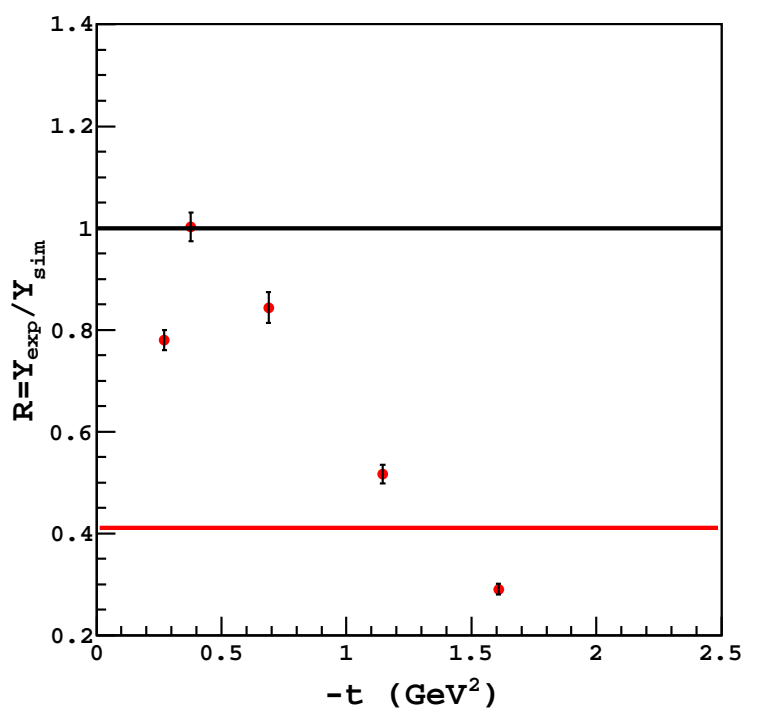

Figure 2.16: The $t$-dependence of the experimental cross sections and the data to Monte Carlo yield ratios, determined using the starting model input cross section described above are shown in panels a and b, respectively, of this figure. The solid red line in panel a) represents the parameterized function describing the extracted experimental cross section and this function was used as a model input cross section for the next iteration. The solid black line in the ratio plot is at unity, which corresponds to the true experimental cross section. The solid red line is the best fit line for the ratios. (Color Online)

is assumed to follow the phase-space factor, $\left(W^{2}-M_{p}^{2}\right)^{2}$ (where $M_{p}$ is the proton mass), based on analyses of the experimental data from Refs. [36, 37], while the $Q^{2}$-dependence was taken as $\left(Q^{2}\right)^{-3}$, based on the scaling study of the prior pion electroproduction transverse cross sections, $\sigma_{T}[11,38]$ done in Ref. [39], since $\sigma_{L}$ drops quite rapidly with increasing $-t$ $[39,40,41]$. The model was optimized to the nominal $Q^{2}$ of $2.4 \mathrm{GeV}^{2}$ and $W$ of $2.0 \mathrm{GeV}$ in an iterative fitting procedure to match the $t$-dependence of the experimental data. As shown in Figure 2.17, the final cross section parameterization for the exclusive $\pi^{+}$electroproduction at high $-t$ is given by a sum of two $t$-dependent exponential functions as,

$$
\frac{d^{2} \sigma}{d t d \phi}=0.568 \cdot e^{-5.676 \cdot|t|}+0.335 \cdot e^{-1.117 \cdot|t|}
$$

This model cross section is valid in the range of $-t$ between 0.272 and $2.127 \mathrm{GeV}^{2}$. The 
significance of $|t|$ coefficients, 5.676 and $1.117 \mathrm{GeV}^{-2}$, are further elaborated in the final chapter of this work.

a)

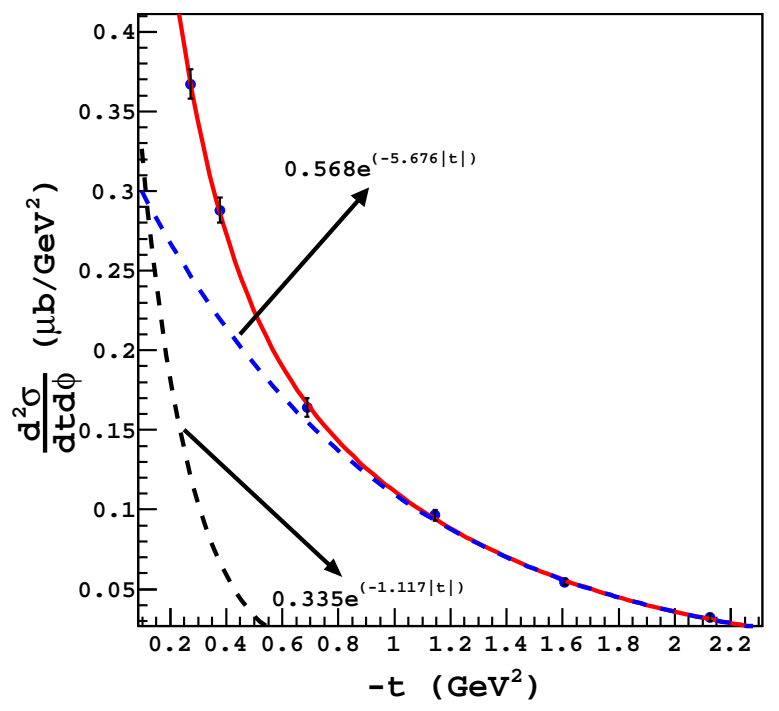

b)

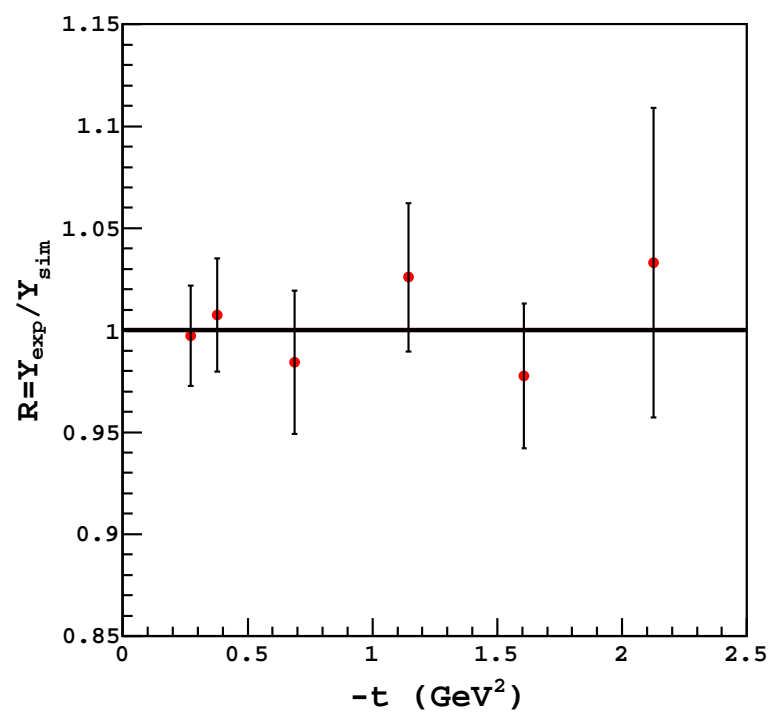

Figure 2.17: In panel a, the extracted experimental cross section versus $-t$ is shown and the data to Monte Carlo yield ratios versus $-t$ is shown in panel b. The solid black line in the ratios plot represents the best fit line, which is at unity. Thus, the extracted cross section in panel a is the final experimental unseparated cross section for this exclusive pion electroproduction at high $-t$ data. The solid red line in panel a is a fit to the experimental cross sections, given by Equation 2.12, whereas the dotted lines represent the breakdown the parameterized function described by the solid red lines into two exponentially decaying functions, given by equations labeled in the plot. Note that the error bars (also in Figure 2.16) include statistical uncertainties only; the systematic uncertainties are discussed in Subsec. 2.4.3. (Color Online)

The unseparated cross sections, extracted following the methodology discussed earlier, is, in principle, dependent on the model cross section; therefore, there is a model dependent systematic uncertainty associated with the extracted $\sigma^{e x p}$. In order to study this uncertainty, a $\phi$-dependence was introduced to the model cross section and the same iterative procedure was applied. The interference terms, $\sigma_{L T}$ and $\sigma_{T T}$ were determined, based on T. Horn's parameterization that reproduces the $\mathrm{F}_{\pi^{-}}$(larger $Q^{2}$ ) [10], $\mathrm{F}_{\pi^{-}} 2$ [11] and Brauel [37] separated cross section data, given by Equation 2.13. The $\sigma^{\text {exp }}$ determined with the $\phi$-dependence was then compared with the $\sigma^{e x p}$ parameterized by Equation 2.11 to determine the model 
dependent uncertainties.

$$
\begin{aligned}
& \frac{d \sigma_{L T}}{d t}=\frac{16.533}{\left(1+Q^{2}\right)} \cdot e^{(-5.1437 \cdot|t|)} \cdot \sin \left(\theta^{*}\right), \\
& \frac{d \sigma_{T T}}{d t}=\frac{178.06}{\left(1+Q^{2}\right)} \cdot e^{(-7.1381 \cdot|t|)} \cdot \sin ^{2}\left(\theta^{*}\right) .
\end{aligned}
$$

\subsubsection{Estimate of Uncertainties}

The statistical uncertainties in the unseparated experimental cross sections are determined by the propagation uncertainties in $Y_{\exp }$ and $Y_{\text {sim }}$ in Equation 2.7. The statistical uncertainty in $Y_{\text {exp }}$ is largely dominated by the uncertainty in the number of measured real events. The $Y_{\text {exp }}$ are calculated using Equation 2.3 and the uncertainties in the total efficiency, $\varepsilon_{\text {total }}$, and in the total accumulated beam charge, $Q_{\text {total }}$, also contribute to the statistical uncertainties in $Y_{\text {exp }}$. The fractional uncertainty in $Q_{t o t a l}$ is $0.5 \%$ for all the $-t$ settings, while the relative uncertainty in $\varepsilon_{\text {total }}$ is less than $2 \%$. Similarly, the only factor contributing to the statistical uncertainty in $Y_{\text {sim }}$ is the uncertainty in the number of simulated real events after applying all the analysis cuts. The statistical uncertainties in both the yield ratio $(R)$ and the experimental unseparated cross sections $\left(\sigma^{e x p}\right)$ range from $2 \%$ to $4 \%$ for all the settings except at the highest $-t$; for that setting, the statistical uncertainty is close to $6 \%$.

The systematic uncertainties are subdivided into two categories: correlated and uncorrelated systematic uncertainties. The correlated (or scale) uncertainties, i.e., those that are the same for all the $-t$ settings, are the ones largely due to the radiative corrections, pion absorption, pion decay and target thickness corrections. Each of the correlated systematic uncertainties are added in quadrature to determine total correlated systematic uncertainty of $3-4 \%$.

The uncorrelated uncertainties, also called random or point-to-point uncertainties, af- 


\begin{tabular}{|c|c|c|c|}
\hline Corrections & $\begin{array}{c}\text { Uncorrelated } \\
\text { (Random) } \\
(\%)\end{array}$ & $\begin{array}{c}\text { Correlated } \\
\text { (Scale) } \\
(\%)\end{array}$ & References \\
\hline Acceptance & 0.6 & 1.0 & Subsec. 2.3 .2 \\
\hline$M_{x}$ cut dependence & 0.5 & & Subsec. 2.4 .3 \\
\hline Model dependence & 4.7 & & Subsec. 2.4 .2 \\
\hline Radiative corrections & & 2.0 & Subsec. 2.3.1 \\
\hline Pion absorption & & 2.0 & Subsec. 2.2 .5 \\
\hline Pion decay & 0.03 & 1.0 & Subsec. 2.3.1 \\
\hline Charge & & 0.5 & Subsec. 2.1.2 \\
\hline Target thickness & & 0.9 & Subsec. 2.2.3 \\
\hline HMS tracking & & 1.0 & Ref. $[11,12]$ \\
\hline SOS tracking & & 0.5 & n \\
\hline CPU dead time & & 0.2 & $"$ \\
\hline HMS trigger & & 0.1 & $"$ \\
\hline SOS trigger & & 0.1 & $"$ \\
\hline Electronics dead time & & 0.3 & Ref. [3] \\
\hline Coincidence blocking & & 0.1 & Subsec. 2.4 .3 \\
\hline$d \theta_{e}$ & 0.1 & & Ref. [3] \\
\hline$d E_{\text {beam }}$ & 0.1 & & " \\
\hline$d p_{e}$ & 0.1 & & $"$ \\
\hline$d \theta_{\pi}$ & 0.1 & & $"$ \\
\hline Total & 4.8 & 3.5 & \\
\hline
\end{tabular}

Table 2.5: Summary of systematic uncertainties in the experimental cross sections. The first column contains all the factors contributing to the systematic uncertainties, which are further broken down into uncorrelated (random) systematic uncertainties in the second column and correlated systematic uncertainties in the third column. The total in the last row of the table is calculated by adding relevant uncertainties quadratically. The final column lists the sections or the references where the various items are discussed.

fect each $-t$ setting independently. For this analysis, the fully uncorrelated uncertainty is dominated by model dependence, resulting in a total uncorrelated uncertainty of $4.8 \%$. As discussed earlier, the experimental cross section was re-calculated using a $\phi$-dependent model (see Equation 2.13) and the resultant changes were evaluated to determine the uncertainty. The random uncertainty due to model dependence was $4.7 \%$. Other larger contributions to the uncorrelated uncertainty come from missing mass, $M_{x}$, cut dependence and acceptance. The influence of the uncertainty due to $M_{x}$ cut dependence, which was around $0.5 \%$, was determined by changing the upper limit of the missing mass cut (see Table 2.3) by \pm 20 and 
assessing the changes in the cross sections. The uncertainties in acceptance are based on extensive single-arm elastic and deep-inelastic measurements on ${ }^{1} \mathrm{H}\left(e, e^{\prime} p\right)$ data $[3,32,42]$ and how well the sieve slit data are reproduced by the used optical matrix elements. The acceptance contributes $0.6 \%$ to the uncorrelated uncertainty and $1.0 \%$ to the correlated uncertainty. The point-to-point uncorrelated systematic uncertainty was added in quadrature with the statistical uncertainty to give total random uncertainty (Table 2.6).

\subsection{Results}

In this section, the results of the analysis are presented. The unseparated experimental cross sections, $\sigma_{U}$, listed in Table 2.6 have been extracted with the help of the Monte Carlo simulation, SIMC, using the relation in Equation 2.7 with the model cross section given by Equation 2.12 and the analysis cuts of Table 2.3. The cross sections are presented for the average values, $\bar{W}$ and $\overline{Q^{2}}$, and bin centered nominal values of $-t$. In the following subsections, the cross section results shown in Table 2.6 will be compared to prior pion electroproduction cross sections data as well as two theoretical models.

\begin{tabular}{cccc}
\hline $\begin{array}{c}|t| \\
\left(\mathrm{GeV}^{2}\right)\end{array}$ & $\begin{array}{c}\overline{Q^{2}} \\
\left(\mathrm{GeV}^{2}\right)\end{array}$ & $\begin{array}{c}\bar{W} \\
(\mathrm{GeV})\end{array}$ & $\begin{array}{c}\sigma_{U} \\
\left(\mu \mathrm{b} / \mathrm{GeV}^{2}\right)\end{array}$ \\
\hline \hline 0.272 & 2.402 & 2.039 & $0.367 \pm 0.020,0.013$ \\
0.378 & 2.427 & 2.029 & $0.288 \pm 0.016,0.010$ \\
0.688 & 2.449 & 2.018 & $0.164 \pm 0.010,0.006$ \\
1.145 & 2.427 & 2.029 & $0.096 \pm 0.006,0.003$ \\
1.608 & 2.433 & 2.020 & $0.054 \pm 0.003,0.002$ \\
2.127 & 2.423 & 2.026 & $0.032 \pm 0.003,0.001$ \\
\hline \hline
\end{tabular}

Table 2.6: Unseparated cross sections, $\frac{d^{2} \sigma}{d t d \phi}\left(\right.$ or $\left.\sigma_{U}\right)$, at $\phi \approx \pi$ for the $p\left(e, e^{\prime} \pi^{+}\right) n$ reaction at high $-t$. The results are presented in the lab frame for the nominal $-t$ values. The kinematic quantities, $\overline{Q^{2}}$ and $\bar{W}$, are the weighted averages after applying all the analysis cuts. The two uncertainies given for $\sigma_{U}$ are the combination of statistical and uncorrelated systematic uncertainties added in quadrature, and the correlated (scale) uncertainty. 


\subsubsection{Comparison with prior data}

The cross section results are compared to two of the prior exclusive pion electroproduction experiments: $\mathrm{F}_{\pi}-2$ and Hall $\mathrm{B}$ data at high $-t$. The second pion form factor experiment, $\mathrm{F}_{\pi^{-}}$, was carried out in 2003 with the aim to increase the $Q^{2}$ range of pion form factor from 1.6 to $2.5 \mathrm{GeV}^{2}$. In order to extract the form factor, first the separated cross sections (L, T, LT, TT) have to be determined using the unseparated cross sections at two different $\varepsilon$ values. As shown in panel a of Figure 2.18, the extracted unseparated cross sections at two lowest $-t$ settings of 0.272 and $0.378 \mathrm{GeV}^{2}$ were compared to the $\mathrm{F}_{\pi}-2$ data at near-parallel kinematics, corresponding to $-t$ values of 0.228 and $0.365 \mathrm{GeV}^{2}$, respectively. The $\mathrm{F}_{\pi}-2$ data compared were at average values, $\left(\overline{Q^{2}}, \bar{W}\right)$ of $\left(2.539 \mathrm{GeV}^{2}, 2.181 \mathrm{GeV}\right)[3]$.

Exclusive electroproduction of $\pi^{+}$above the resonance region was studied using CEBAF Large Acceptance Spectrometer (CLAS) at Jefferson Lab by scattering a $6 \mathrm{GeV}$ continuous electron beam off a proton target. The unseparated cross sections were measured for kinematics region: $0.16<x_{B}<0.58,1.6<Q^{2}<4.5 \mathrm{GeV}^{2}$ and $0.1<-t<5.3 \mathrm{GeV}^{2}[15]$. For the comparison, the data set close to the kinematics of this high $-t$ measurement was used. The CLAS data compared span the $-t$ range from 0.85 to $4.50 \mathrm{GeV}^{2}$ at $Q^{2}=2.65$ $\mathrm{GeV}^{2}, W=2.1 \mathrm{GeV}$, and $\varepsilon$ and $x_{B}$ of 0.56 and 0.37 , respectively. As shown in panel a of Figure 2.18, the CLAS data agree with the unseparated cross sections determined with this analysis within the uncertainties. Note that both data sets used for comparison had to be scaled to $Q^{2}$ and $W$ of $2.40 \mathrm{GeV}^{2}$ and $2.0 \mathrm{GeV}$, respectively, using the same scaling relations discussed in Subsec. 2.4.2.

\subsubsection{Theoretical models}

In addition to comparing the results from this analysis to the previous data, they were also compared with two different theoretical models available. The first model used for 
a)

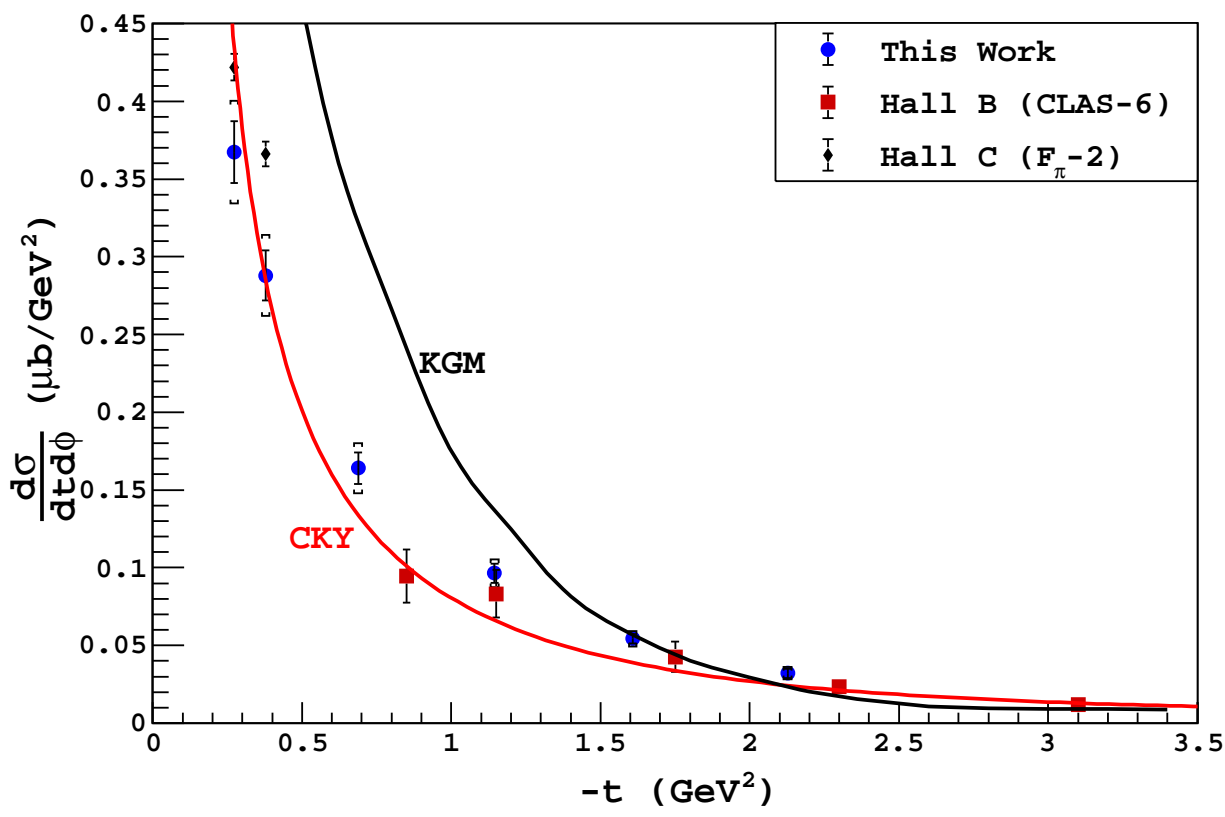

b)

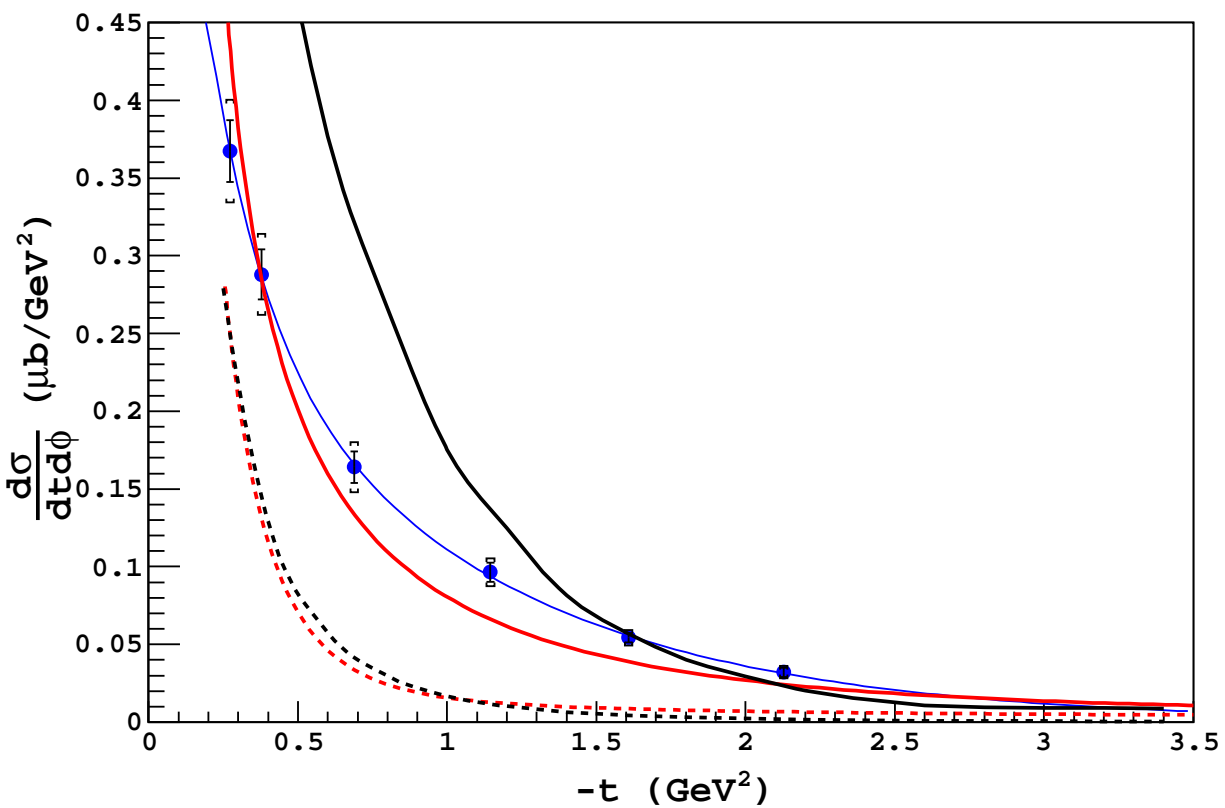

Figure 2.18: Unseparated cross section versus - $t$. In panel a, unseparated cross section versus $-t$ is shown is compared with prior data from $\mathrm{F}_{\pi}-2$ and CLAS collaboration, which are shown in black and red data points, respectively. The blue data points, consisting of two error bars, are the results from this work. The smaller error bar represent the random (statistical and uncorrelated systematics added in quadrature) uncertainties and the difference between the two error bars represent the correlated systematic uncertainties. The solid blue line represents the model cross section, which reproduces the cross section results from this analysis. In panel b, log plot comparing the extracted unseparated cross sections with the two models is shown. The solid red and black lines (in both panels) represent the CKY and KGM models, respectively. The dotted red and black lines (in panel b only) show the longitudinal contributions to the respective model cross sections. (Color Online) 
comparison is the Regge-based model developed by T. K. Choi, K. J. Kong, and B. G. Yu, the so-called "CKY" model. The results are also compared to the "hybrid" model proposed by M. Kaskulov, K. Gallmeister, and U. Mosel, the so-called "KGM" model, which taken into account both hadronic and partonic components. These models are briefly described in the following subsections.

\section{CKY model}

In Ref. [41], Choi, Kong, and Yu (CKY) developed a Regge-based model for pion electroproduction, in which pole-like propagators of Born term models are replaced with Regge propagators. In other words, the interaction in this model is effectively described by $\pi+\rho$ Regge trajectory exchanges. Analyses of electroproduction data have shown that the cross sections of the process are largely determined by the pion and nucleon electromagnetic form factors that include information about the hadron structure [40, 41, 43]. This model is a modification of VR-type model (see Subsec. 3.3.1), mostly in the treatment of form factors. In the VR model, the charge form factor is described using a simple dipole-like form. For instance, the proton charge form factor in the VR model is given by [40],

$$
F_{p}\left(Q^{2}, s\right)=\frac{1}{\left(1+Q^{2} / \Lambda_{\gamma p p^{*}}^{2}\right)^{2}},
$$

where the cutoff mass $(\Lambda)$ has an energy, $s$, dependence. Unlike the VR model, where the cutoff mass is fixed, in the CKY model, this parameter, $\Lambda$, for pion or nucleon form factors can be adjusted to fit to cross sections. The model describes the prior DESY and CLAS data at high $Q^{2}$ and $-t$ pretty well and is valid up to $-t \approx 5.0 \mathrm{GeV}^{2}$ at large $Q^{2}$ and high invariant mass, $W$ [41]. The comparison between the cross section results from this analysis and the CKY model is done in Figure 2.18. In panel a, the CKY model in solid red line describes the unseparated cross section very well, both at low and high $-t$ regions. The 
agreement is better at high $-t$ but the model also describes the low $-t$ region fairly well. The longitudinal component of the cross sections using the CKY model is shown in panel b of the same figure as a dotted red line.

\section{KGM model}

The second model with which these high $-t$ cross section results are compared is the "hybrid" two-component hadron-parton model proposed in Ref. [44]. Similar to the VR and CKY models discussed earlier, the KGM model is also based on the $\pi+\rho$ Regge trajectory exchanges in the $-t$ channel. However, the model complements the soft hadron-like interactions, which dominate in photoproduction and low $Q^{2}$ electroproduction, by direct hard interactions of virtual photons with partons, followed by the hadronization process into $\pi^{+} n$ at higher values of $Q^{2}$ [44]. The partonic part of the production mechanism is described by a "deep-inelastic" scattering (DIS) type electroproduction mechanism, where the quark knockout reaction, $\gamma^{*} q \rightarrow q$, is followed by the quark fragmentation process of the Lund type $[15,44]$. Figure 2.18 shows the results of this model compared to these data as a solid black line, where a good agreement was found at higher values of $-t\left(>1.0 \mathrm{GeV}^{2}\right)$. The dotted black line in panel b of Figure 2.18 shows the longitudinal component of the cross section given by the model. The discussion on the results, and comparisons as well as some parting remarks are given in the final chapter of this work. 


\section{Chapter 3}

\section{Projections for $p\left(e, e^{\prime} K^{+}\right) \Lambda, \Sigma^{0}$}

\section{Experiment}

The primary goal of the upcoming exclusive $K^{+}$electroproduction experiment, E1209-011, is to measure, for the first time, separated cross sections (L,T,TT,LT) for the $p\left(e, e^{\prime} K^{+}\right) \Lambda\left(\Sigma^{0}\right)$ reactions using the Rosenbluth separation technique [14]. With the measurement of the L-T separated exclusive $K^{+}$electroproduction cross sections, we will have the unique opportunity to elucidate the reaction mechanism underlying strangeness production by studying the $Q^{2}$ and $t$-dependences of the cross sections. Moreover, the transition from hadronic to partonic degrees of freedom in exclusive processes can also be studied using separated cross sections via QCD scaling studies. This chapter focuses on projection studies for the E12-09-011 experiment. The chapter begins with an overview of the experimental setup, particle identification (PID) method, as well as the proposed kinematics. In addition to a brief discussion on the two theoretical models used for comparison in the simulation, the chapter also describes, in detail, the analysis of the simulated data in order to estimate projected real and dominant backgrounds for $\Lambda$ and $\Sigma^{0}$ final states. 


\subsection{Experimental Overview}

Even with many decades of effort $[45,46,47,48,49,50]$, a thorough description of the reaction mechanism underlying strangeness production from a proton above the resonance region is not available. This plight, in comparison to the pion production $[3,11,12]$, can mainly be imputed to the experimental challenges encountered in kaon electroproduction. For instance, the kaon production cross section is much smaller, and kaon PID is more challenging due to the shorter lifetime, and separation of kaons from pion and proton backgrounds. With the higher beam energies and a high luminosity spectrometer system consisting of the new Super High Momentum Spectrometer (SHMS) and the well studied and understood High Momentum Spectrometer (HMS) at Hall C, we have the opportunity to drastically improve upon this situation.

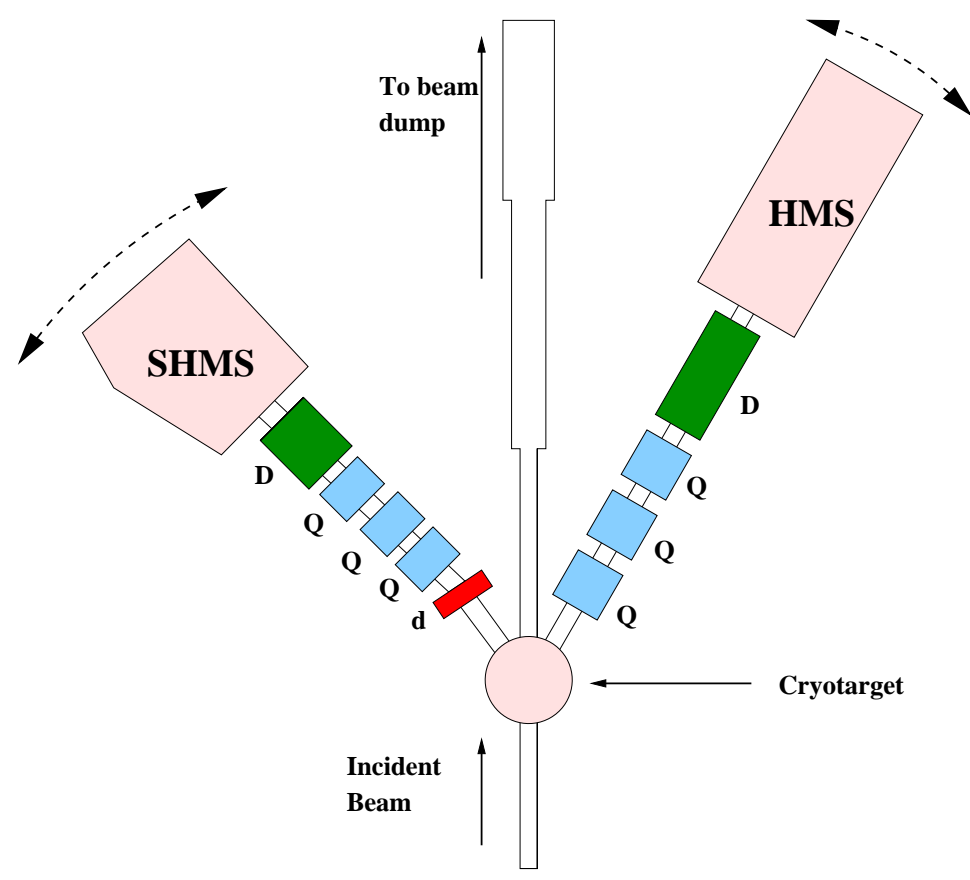

Figure 3.1: Schematic top view of the Jefferson Lab Hall C spectrometers relative to the target and the beamline.

The $p\left(e, e^{\prime} K^{+}\right) \Lambda, \Sigma^{0}$ experiment will be performed in Hall $\mathrm{C}$ at Jefferson Lab. The schematic top view of the experimental setup is illustrated in Figure 3.1, where the unpo- 
larized continuous electron beam from the Jefferson Lab accelerator will be incident on a liquid hydrogen $\left(\mathrm{LH}_{2}\right)$ target situated in a vacuum scattering chamber. The electroproduced kaon will be detected in the SHMS, in coincidence with the scattered electron in the HMS. The individual detectors are housed in concrete-shielded detector huts in either spectrometer. The signals from the detectors in the experimental hall are sent to remote electronics situated in the Hall $\mathrm{C}$ counting house, where the data are processed and recorded by the data acquisition (DAQ) system.

\subsubsection{Spectrometers}

For this experiment, the HMS will be configured for electron detection, whereas the new spectrometer SHMS that replaced the old Short Orbit Spectrometer (SOS) will detect kaons. The configuration of the magnets for the SHMS is nearly identical to the HMS, with three super-conducting quadrupole magnets $(Q 1, Q 2, Q 3)$ and one vertically bending dipole magnet $(D)$, as can be seen in Figure 3.1. The quadrupoles are used for focusing the particles into the dipole, improving the solid angle acceptance of the spectrometer and the dipole is used to disperse the incoming particles according to their charge and momentum. Apart from the standard $Q Q Q D$ magnet configuration, the SHMS is also equipped with an additional super-conducting horizontal bending magnet $(d)$ in order to achieve a smaller scattering angle. It is located in front of the $Q 1$ and behind the target station, and is capable of bending produced charged particles by $3^{\circ}$. With this additional bending magnet $d$, the optical configuration of the SHMS becomes $d Q Q Q D$.

A high luminosity spectrometers system, like the SHMS+HMS combination in Hall C, is well-suited for these measurements, since the magnetic spectrometers benefit from relatively small point-to-point uncertainties, which are crucial for a meaningful L-T separation. In particular, the optical properties and the acceptance of the HMS have been studied exten- 


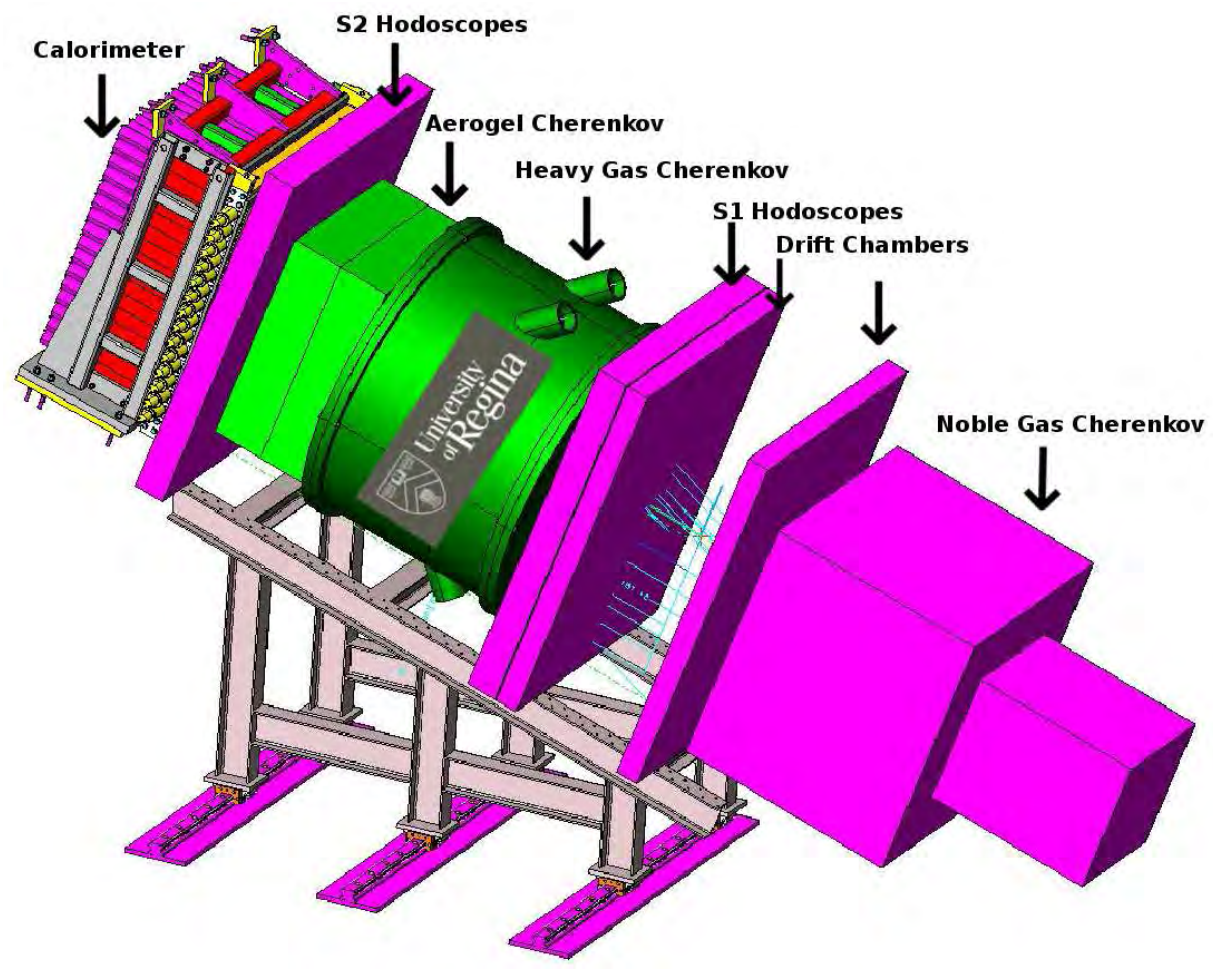

Figure 3.2: Schematic side view of the SHMS detector package [16].

\begin{tabular}{lcr}
\hline \multicolumn{1}{c}{ Quantity } & HMS & SHMS \\
\hline \hline Dipole Bend Angle $\left(^{\circ}\right)$ & 25.0 & 18.4 \\
Central Momenta Range $(\mathrm{GeV} / \mathrm{c})$ & $0.5-7.5$ & $2.0-11.0$ \\
Focal Length $(\mathrm{m})$ & 26.0 & 18.1 \\
Scattering Angle Range $\left(^{\circ}\right)$ & $12.5-90$ & $5.5-40.0$ \\
Momentum Acceptance $(\delta p / p)$ & $\pm 10 \%$ & $-10 \%<\delta<+22 \%$ \\
Momentum Resolution & $<0.1 \%$ & $0.03-0.08 \%$ \\
Solid Angle Acceptance $(\mathrm{msr})$ & 6.7 & 4.0 \\
Horizontal Acceptance $(\mathrm{mrad})$ & \pm 27.5 & \pm 24.0 \\
Vertical Acceptance $(\mathrm{mrad})$ & \pm 70.0 & \pm 40.0 \\
Horizontal Resolution $(\mathrm{mrad})$ & 0.8 & $0.5-1.2$ \\
Vertical Resolution $(\mathrm{mrad})$ & 0.9 & $0.3-1.1$ \\
Maximum DAQ Rate & $\sim 10000$ events/second & $\sim 10000$ events/second \\
Target Vertex Length $(\mathrm{cm})$ & \pm 7.0 & \pm 15.0 \\
Target Vertex Reconstruction Accuracy $(\mathrm{mm})$ & 1.0 & $0.1-0.3$ \\
\hline
\end{tabular}

Table 3.1: The performance and design parameters comparison between HMS and SHMS [16]. 
sively and are very well understood in the kinematic range between 0.5 to $7.5 \mathrm{GeV} / \mathrm{c}$. The HMS detector package consists of a pair of drift chambers which provides the position and trajectory information of a particle, Aerogel C̆erenkov Detector (ACD) to provide adequate hadron identification at central momenta above $3 \mathrm{GeV} / \mathrm{c}$, two pairs of plastic scintillator hodoscopes to provide the raw trigger for the data acquisition, Heavy Gas Cerenkov Detector (HGC) for particle separation, and Lead Glass Calorimeter as an additional way of separating particles as discussed in Chapter 2. Both the spectrometers, HMS and SHMS, make use of similar detectors. However, the SHMS detector package consists of an additional removable Noble Gas Cerenkov Detector (NGC) with the main aim being to separate electrons from heavier charged particles at high central momenta above $6 \mathrm{GeV} / \mathrm{c}$. Furthermore, one of the planes in S2 (see Figure 3.2) hodoscopes in the SHMS contains quartz bars instead of scintillator, relying on the Čerenkov process rather than scintillation. The complete detector package for SHMS is shown in Figure 3.2. For this experiment, the NGC in the SHMS will be removed and replaced with a vacuum tank of the same length to eliminate sources for multiple scattering. The HMS HGC and calorimeter will be used for electron identification and the SHMS hodoscopes, Aerogel and Heavy Gas Čerenkov detectors will be used for the $K^{+}$particle identification, where the hodoscopes and HGC will be used for the pion/kaon separation and the ACD will provide the proton/kaon separation. The important performance specifications comparing both spectrometers are shown in Table 3.1.

\subsection{Proposed Kinematics}

The kinematics of the $p\left(e, e^{\prime} K^{+}\right) \Lambda\left(\Sigma^{0}\right)$ reactions in the laboratory frame are shown in Figure 3.3. The incident electron with four momentum $k=\left(\varepsilon_{k}, \mathbf{k}\right)$ interacts with the nucleon via virtual photon exchange. The scattering plane is defined by three momentum vectors of the incoming and outgoing electrons, given by $\mathbf{k}$ and $\mathbf{k}^{\prime}$ respectively, and the electron 
scattering angle is denoted by $\theta_{e}$. The four-momentum transferred by the electron to the photon-nucleon system is given by $q=(\omega, \mathbf{q})$, where $\mathbf{q}=\mathbf{k}-\mathbf{k}^{\prime}$ and $\omega=E_{i}-E_{f}$. The virtual photon is absorbed by the target proton and a kaon is emitted with four-momentum $p^{\prime}=\left(E\left(p^{\prime}\right), \mathbf{p}_{\mathbf{K}}\right)$, where $\mathbf{p}_{\mathbf{K}}$ is oriented relative to the scattering plane by a polar angle $\theta_{K q}$ and an azimuthal angle $\phi_{K q}$.

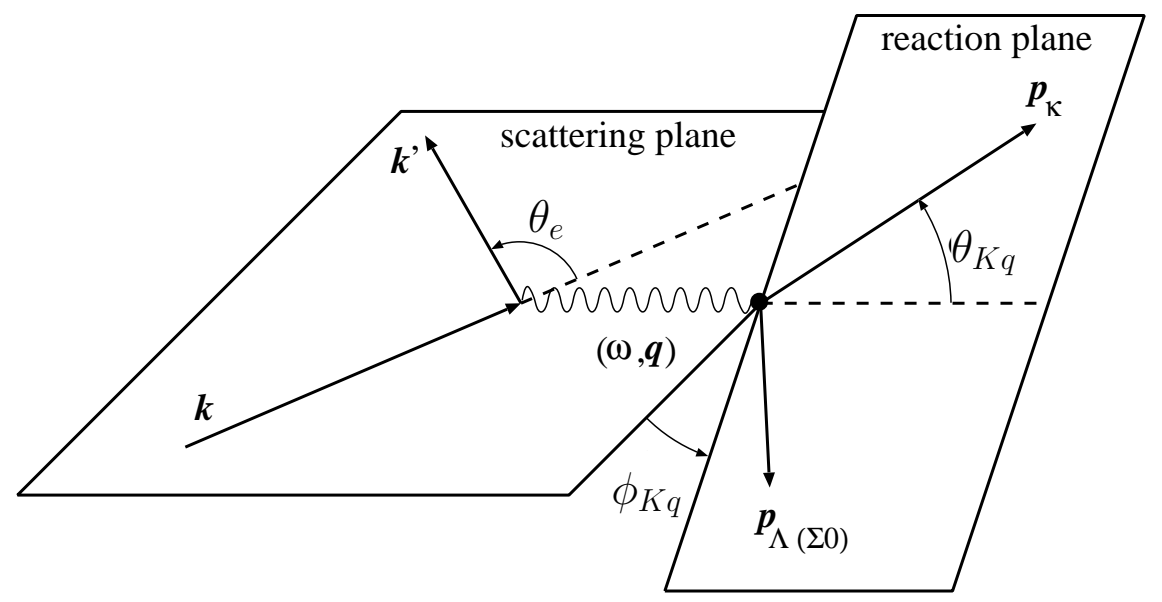

Figure 3.3: Schematic diagram of the $p\left(e, e^{\prime} K^{+}\right) \Lambda, \Sigma^{0}$ reaction in the lab frame. The scattering plane is defined by the three-vectors of the initial and final electron. The momentum transferred to the photon-nucleon system is defined in terms of the four-momentum transfer, $Q^{2}$. The reaction plane is defined by the three-vectors of the produced kaon and the recoiling nucleon [51].

The kinematics for the exclusive $K^{+}$electroproduction reaction are selected keeping in mind the two-fold scientific goals for the experiment, which were discussed in Chapter 1. In order to study the reaction mechanism, the $t$-dependence of separated cross sections will be measured at fixed $Q^{2}$, and if the evidence of $K^{+}$pole dominance is seen in the longitudinal cross section, the $Q^{2}$-dependence of the kaon form factor will also be measured above the resonance region for the first time. Additionally, the hard $\mathrm{QCD} Q^{-n}$ scaling tests will be performed by examining the $Q^{2}$-dependence of the cross section at two fixed values of $x_{B}$. The accessible $Q^{2}-\mathrm{x}_{B}$ phase space for the experiment is illustrated in Figure 3.4. The $Q^{2}$ coverage for this experiment is roughly a factor of three larger compared to the $4 \mathrm{GeV}$ kaon electroproduction data [2] at small $-t$ since the beam energies available for the $12-\mathrm{GeV}$ era 
Jefferson Lab are higher. The proposed measurements for this experiment will provide the first data in the region above the dominant resonances, as shown in Figure 3.4, which will allow for a more reliable interpretation of the $-t$ and $Q^{2}$ dependences of $\sigma_{L}$ and $\sigma_{T}$ with access of higher values of $W$.

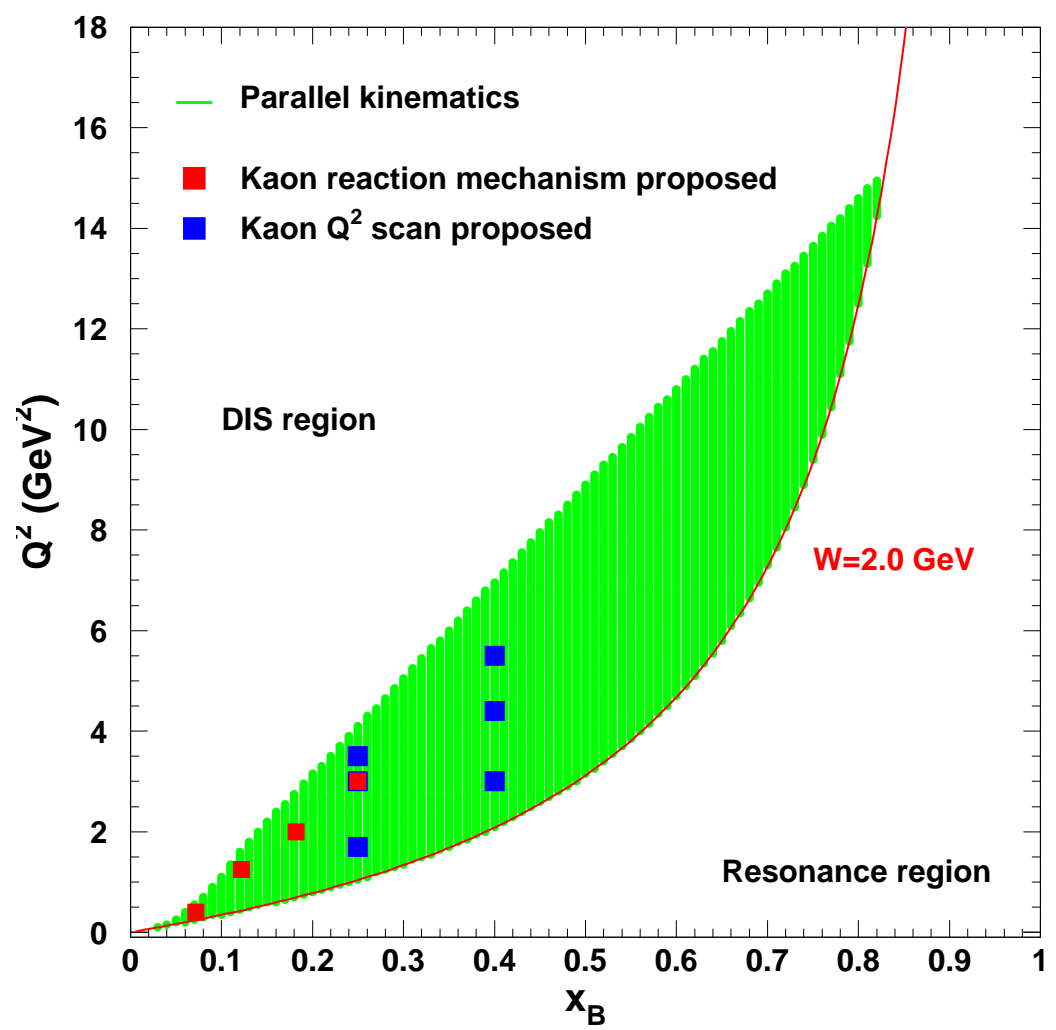

Figure 3.4: $Q^{2}-x_{B}$ phase space available for L-T separation in Hall C at $12 \mathrm{GeV}$ using the $H M S+S H M S$ combination. The $Q^{2}$-dependence of the longitudinal cross section will be measured at two different values of $x_{B}$ of 0.25 and 0.40 and the relevant settings are denoted by blue data points in the plot. The settings represented by red data points will be used to study the $t$ dependence of the longitudinal cross section for $Q^{2}$ ranging from 0.40 to 3.00 $\mathrm{GeV}^{2}[51]$.

The complete kinematics coverage, showing all the relevant kinematic quantities for the E12-09-011 experiment, are listed in Table 3.2. The kinematics settings pertaining to the study of the reaction mechanism and to the hard QCD scaling study are clearly labeled in the table. The data point at $Q^{2}=3.0 \mathrm{GeV}^{2}$ and $x_{B}=0.25$ is optimized to be used for both 
studies. All the proposed kinematic settings use beam energies with standard linac gradients of $2.20 \mathrm{GeV}$ per pass, except for the lower $Q^{2}$ settings of $0.40,1.25$, and $2.00 \mathrm{GeV}^{2}$, which require special linac tunes to achieve the necessary beam energies. The charged kaons will be detected by the SHMS in near-parallel kinematics $\left(\theta_{K q}\right.$ near zero), which allows for a separation of each of the cross section components over a limited $-t$ range. A minimum of two beam energies are required to be able to extract $\sigma_{L}$ and $\sigma_{T}$ from the data, hence the measurement will be taken at two different values of $\varepsilon$, keeping the rest of the Lorentz invariant kinematic variables the same. The two $\varepsilon$ settings are chosen in such a way that $\Delta \varepsilon \geq 0.20$, where possible, to keep the amplification of the systematic uncertainty to a minimum. For the $Q^{2}=2.0 \mathrm{GeV}^{2}$ setting, the data will be taken at three different beam energies instead of two, to provide a consistency check in the extraction of $\sigma_{L}$ and $\sigma_{T}$.

In order to perform a complete separation of the L, T, LT, and TT terms over a wider $-t$ range, the data will be acquired not only along the $\vec{q}$ vector but also $3^{\circ}$ to the left and right of $\vec{q}$ vector. This will provide a wider $|t|-\phi_{K}$ coverage, which in turn will make the extraction of cross section interference terms, $\sigma_{L T}$ and $\sigma_{T T}$ possible with the study of the $\phi_{K}$-dependence of the cross section. An example polar plot of $|t|-\phi_{K}$ coverage, including all three angle settings, is shown in Figure 3.5.

The study of $-t$ dependence will be done at $Q^{2}=0.40,1.25,2.00$, and $3.00 \mathrm{GeV}^{2}$. First, the L-T-LT-TT separated cross sections will be determined, and the contributions of $\sigma_{L}$ and $\sigma_{T}$ to the $\Lambda$ and $\Sigma^{0}$ final states can be examined. This would provide important information about the role of $K$ and $K^{*}$ exchange contributions in the $t$-channel. If the $K$ pole contribution at low $-t$ dominates the $\sigma_{L}$ for the $K^{+} \Lambda$ channel, the data can be used to extract the kaon form factor, $F_{K}$, analogous to the $\pi^{+}$case [51]. If the data allows for the extraction of $F_{K}$, a comparison of the $Q^{2}=0.40 \mathrm{GeV}^{2}$ data taken close to the kaon pole, $-t_{\text {min }}=0.064 \mathrm{GeV}^{2}$, with the elastic kaon form factor results from CERN will help 
cross-check the extracted $F_{K}$ values using the electroproduction method against those obtained without approximation from elastic $e-K$ scattering at the CERN SPS [52]. With the validation of the electroproduction method to extract form factors for the lowest $Q^{2}$ setting, the data at higher values of $Q^{2}$ could possibly provide the first extraction of the kaon form factor above the resonance region.

Similarly, the $Q^{2}$ scans at fixed values of $x_{B}=0.25$ and $x_{B}=0.40$ at fixed $-t$ of 0.2 and $0.5 \mathrm{GeV}^{2}$, respectively, will access the $Q^{2}$ regime between $1.0-5.5 \mathrm{GeV}^{2}$ for the first time above the resonance region. This will allow us to obtain reliable L-T-LT-TT separated

\begin{tabular}{|c|c|c|c|c|c|c|c|c|c|c|}
\hline $\begin{array}{c}Q^{2} \\
\left(\mathrm{GeV}^{2}\right) \\
\end{array}$ & $\begin{array}{c}W \\
(\mathrm{GeV})\end{array}$ & $x_{B}$ & $\begin{array}{c}-t \\
\left(\mathrm{GeV}^{2}\right)\end{array}$ & $\varepsilon$ & $\begin{array}{c}T_{i n c} \\
(\mathrm{GeV})\end{array}$ & $\begin{array}{c}T_{e^{\prime}} \\
(\mathrm{GeV})\end{array}$ & $\begin{array}{c}\theta_{e^{\prime}} \\
(\mathrm{deg})\end{array}$ & $\begin{array}{c}\theta_{q} \\
(\mathrm{deg})\end{array}$ & $\begin{array}{c}P_{k} \\
(\mathrm{GeV} / \mathrm{c})\end{array}$ & $\begin{array}{r}\theta_{k q} \\
(\mathrm{deg})\end{array}$ \\
\hline \multicolumn{11}{|c|}{ Study of the reaction mechanism and form factor $\mathrm{Q}^{2}$ dependence } \\
\hline 0.40 & 2.45 & 0.072 & 0.064 & 0.411 & 3.799 & 0.857 & 20.2 & -5.6 & 2.669 & $0,+3$ \\
\hline 0.40 & 2.45 & 0.072 & 0.064 & 0.685 & 4.951 & 2.008 & 11.5 & -7.7 & 2.669 & $-2.15,0,+3$ \\
\hline 1.25 & 3.14 & 0.122 & 0.084 & 0.492 & 7.495 & 2.044 & 16.4 & -6.0 & 5.189 & $0,+3$ \\
\hline 1.25 & 3.14 & 0.122 & 0.084 & 0.699 & 9.343 & 3.892 & 10.6 & -7.4 & 5.189 & $-1.9,0,+3$ \\
\hline 2.00 & 3.14 & 0.182 & 0.138 & 0.395 & 7.495 & 1.645 & 23.2 & -6.2 & 5.561 & $0,+3$ \\
\hline 2.00 & 3.14 & 0.182 & 0.138 & 0.580 & 8.761 & 2.910 & 16.1 & -7.7 & 5.561 & $-2.2,0,+3$ \\
\hline 2.00 & 3.14 & 0.182 & 0.138 & 0.752 & 10.921 & 5.070 & 10.9 & -9.2 & 5.561 & $-3,0,+3$ \\
\hline 3.00 & 3.14 & 0.250 & 0.219 & 0.391 & 8.191 & 1.807 & 26.0 & -6.9 & 6.053 & $0,+3$ \\
\hline 3.00 & 3.14 & 0.250 & 0.219 & 0.691 & 10.921 & 4.537 & 14.1 & -9.6 & 6.053 & $-3,0,+3$ \\
\hline \multicolumn{11}{|c|}{ Scaling study at fixed $\mathrm{x}_{B}=0.25,-\mathrm{t}=0.2$} \\
\hline 1.70 & 2.45 & 0.249 & 0.239 & 0.595 & 5.647 & 2.012 & 22.3 & -11.4 & 3.277 & $0,+3$ \\
\hline 1.70 & 2.45 & 0.249 & 0.239 & 0.856 & 8.761 & 5.125 & 11.6 & -14.9 & 3.277 & $-3,0,+3$ \\
\hline 3.50 & 3.37 & 0.250 & 0.215 & 0.364 & 9.343 & 1.895 & 25.7 & -6.1 & 7.122 & $0,+3$ \\
\hline 3.50 & 3.37 & 0.250 & 0.215 & 0.557 & 10.921 & 3.473 & 17.5 & -7.8 & 7.122 & $-3,0,+3$ \\
\hline \multicolumn{11}{|c|}{ Scaling study at fixed $\mathrm{x}_{B}=0.40,-\mathrm{t}=0.5$} \\
\hline 3.00 & 2.32 & 0.400 & 0.531 & 0.634 & 6.601 & 2.603 & 24.1 & -14.1 & 3.486 & $0,+3$ \\
\hline 3.00 & 2.32 & 0.400 & 0.531 & 0.888 & 10.921 & 6.923 & 11.4 & -18.4 & 3.486 & $-3,0,+3$ \\
\hline 4.40 & 2.74 & 0.399 & 0.507 & 0.479 & 8.191 & 2.314 & 27.9 & -10.0 & 5.389 & $0,+3$ \\
\hline 4.40 & 2.74 & 0.399 & 0.507 & 0.735 & 10.921 & 5.045 & 16.3 & -13.1 & 5.389 & $-3,0,+3$ \\
\hline 5.50 & 3.02 & 0.400 & 0.503 & 0.372 & 9.343 & 2.021 & 31.3 & -7.9 & 6.842 & $0,+3$ \\
\hline 5.50 & 3.02 & 0.400 & 0.503 & 0.562 & 10.921 & 3.599 & 21.6 & -9.9 & 6.842 & $-3,0,+3$ \\
\hline
\end{tabular}

Table 3.2: Proposed kinematic settings for exclusive $K^{+}$electroproduction above the resonance region. The data points at $Q^{2}=3.0 \mathrm{GeV}^{2}$ and $W=3.14 \mathrm{GeV}$ is optimized so that they can be used for both the study of the reaction mechanism and the scaling studies. This will not only reduce the required beam time, but will also improve the statistical uncertainty for scaling studies, which typically has lower statistics [51]. 


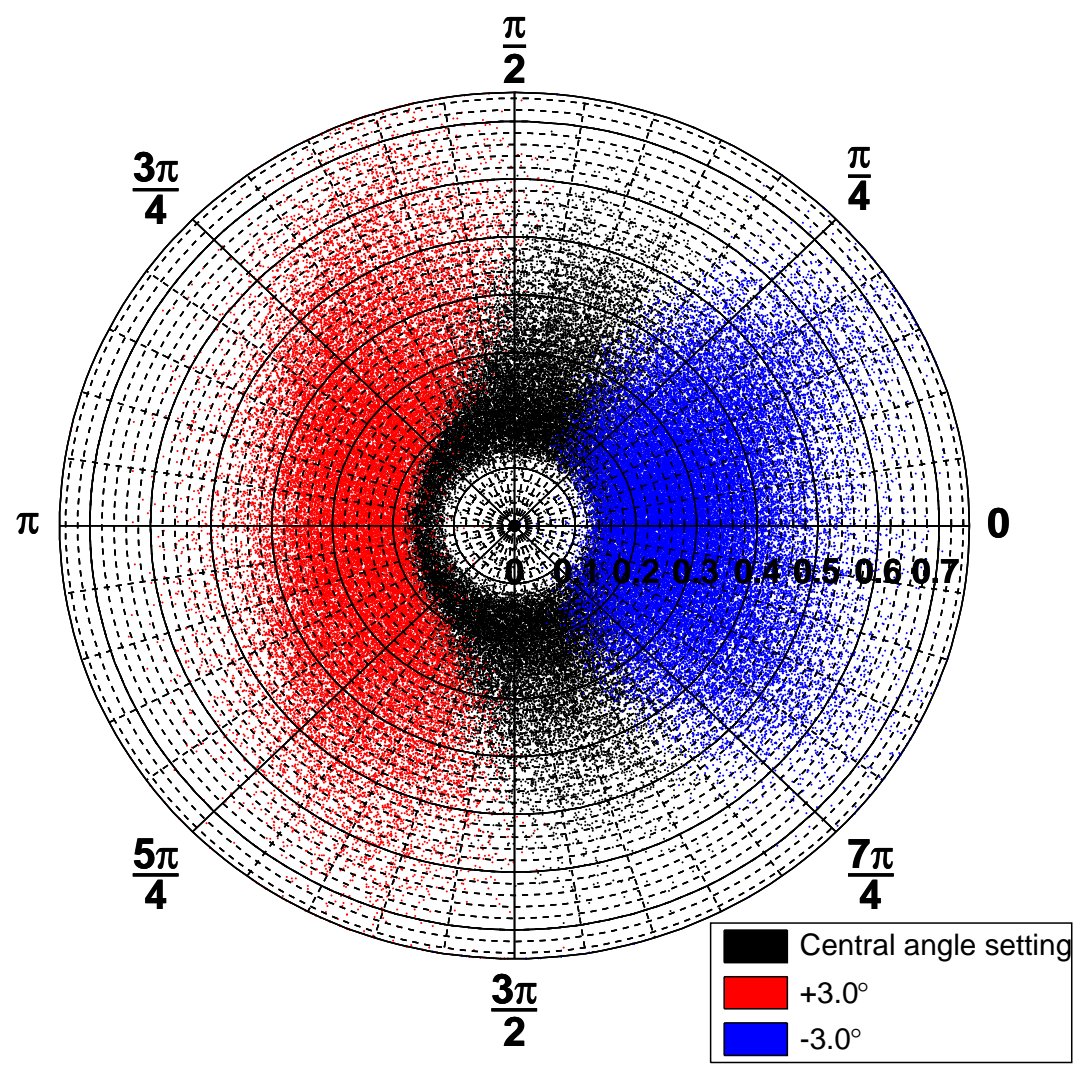

Figure 3.5: Polar plot showing simulated $-t$ versus the azimuthal angle $\phi_{K}$ for the proposed measurement at $Q^{2}=2.0 \mathrm{GeV}^{2}$ setting at high $\varepsilon$. The radial coordinate, $-t$ ranges from 0.0 to $0.75 \mathrm{GeV}^{2}$. The colors indicate the kinematic points with the SHMS set at zero (black), $+3^{\circ}$ (blue), and $-3^{\circ}$ (red) with respect to the $\vec{q}$ vector.

data for the investigations of the onset of $1 / Q^{n}$ scaling in the systems where strangeness is involved. Due to the constraint imposed by the requirement to keep $-t \ll 1.0 \mathrm{GeV}^{2}$, coupled with the maximum available beam energy of the upgraded CEBAF at Jefferson Lab and the kinematic access of the SHMS+HMS configuration in Hall C, the maximum achievable $Q^{2}$ value is close to $10 \mathrm{GeV}^{2}$, where $\Delta \varepsilon$ is kinematically restricted. Thus, as an exploratory measurement, the $Q^{2}$ of $5.5 \mathrm{GeV}^{2}$ is chosen to be the maximum limit for the $Q^{2}$ scan despite the objective of this measurement to extend our knowledge of the relative longitudinal and transverse contributions of the cross section to the largest possible $Q^{2}$. Should the results of this study be sufficiently promising, a follow-up experimental proposal will be submitted to extend these studies. The ratios $R=\sigma_{L} / \sigma_{T}$ are also effectively unknown, and the projected 
ratios based on prior kaon electroproduction data predict a rapid increase of the uncertainties at higher values of $Q^{2}$. However, it should be emphasized that the run-plan requires only minor adjustments to reach a higher value of $Q^{2}$ of $8.0 \mathrm{GeV}^{2}$, if the new data indicate that the uncertainties would be acceptable.

\section{$3.3 \quad$ SIMC}

An overview of the standard Hall C Monte Carlo package (SIMC), which was used for the analysis of various previous Hall $\mathrm{C}$ experiments including $p\left(e, e^{\prime} \pi^{+}\right) n$ at high $-t$, has already been given in Chapter 2. Therefore, this section will only focus on the theoretical models used in SIMC in order to perform the rate estimates for $p\left(e, e^{\prime} K^{+}\right) \Lambda, \Sigma^{0}$ reaction.

\subsubsection{Use of theoretical models in the Projections}

In order to study the real rates for the $p\left(e, e^{\prime} K^{+}\right) \Lambda\left(\Sigma^{0}\right)$ reaction, two models are implemented in SIMC to generate the reaction cross sections. "Old Kaon" is an empirical model based on the fits to previous $K^{+}$electroproduction data for both $\Lambda$ and $\Sigma^{0}$ final states over the $Q^{2}$ range of 0.34 to $0.54 \mathrm{GeV}^{2}$ and the $W$ range of 1.72 to $1.94 \mathrm{GeV}$. The parametrization of the data was originally done by D. Kolentuk in 1999 and it was later improved by T. Horn in 2007 [53].

The second model is based on the calculations of Vrancx and Ryckebusch (VR), namely the VR model. It uses saturation of $\pi$ and $\rho$ Regge trajectories to describe pion electroproduction reactions and features the Reggeized background amplitudes [54]. The VR model is optimized for $Q^{2}$ ranging from 0.2 to $5.0 \mathrm{GeV}^{2}$ and $W$ between 2.0 to $4.0 \mathrm{GeV}$ and generates cross sections for the $\Lambda$ channel [40]. For the $\Sigma^{0}$ channel, the RPR-2007 model is used which, like the VR model, also based on the Regge theory. It makes use of the "Regge plus the 
resonance" approach to determine reaction cross sections [43]. The simulated yields from the "Old Kaon" and VR models will be compared in Section 3.5.

\subsection{Data Analysis}

This section is devoted to the analysis of the simulated data, with the end goal of calculating the real and accidental coincidence rates for each kinematic setting of the exclusive $K^{+}$electroproduction experiment. It begins with a short discussion on the acceptance of each of the spectrometers, followed by a study of the $Q^{2}-W$ distributions at high and low $\varepsilon$ settings. Then, the $\Lambda$ and $\Sigma^{0}$ final states are separated by examining the missing mass distributions of the corresponding final states. The section ends with an overview of all the analysis cuts used for the calculation of the expected real and random coincidence rates.

\subsubsection{Spectrometer Acceptance}

The spectrometer acceptance is a function of the target quantities $\left(x_{t a r}^{\prime}, y_{t a r}^{\prime}, y_{t a r}, \delta\right)$. These quantities are reconstructed using the focal plane quantities $\left(x_{f p}, y_{f p}, x_{f p}^{\prime}, y_{f p}^{\prime}\right)$ and a set of reconstruction matrix elements. The focal plane quantities are determined by fitting a straight line track to the simulated wire chamber hits [2], while the reconstruction matrix is simply the inverse of the forward COSY model matrix (see Section 2.3). While generating events in SIMC, the spectrometer acceptance are established by the input to the simulation. Normally such inputs are chosen in such a way that they slightly exceed the physical acceptance of the spectrometers.

The spread of reconstructed target quantities from the simulation are examined first, which are illustrated in Figure 3.6. Since the acceptance limits used in the simulation surpass the physical acceptance of the spectrometers, cuts on the target quantities are required in 
a)

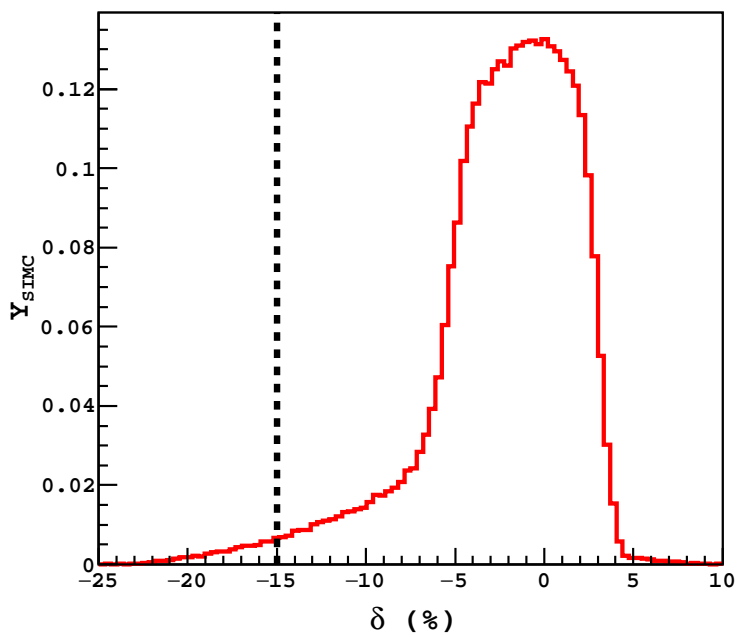

c)

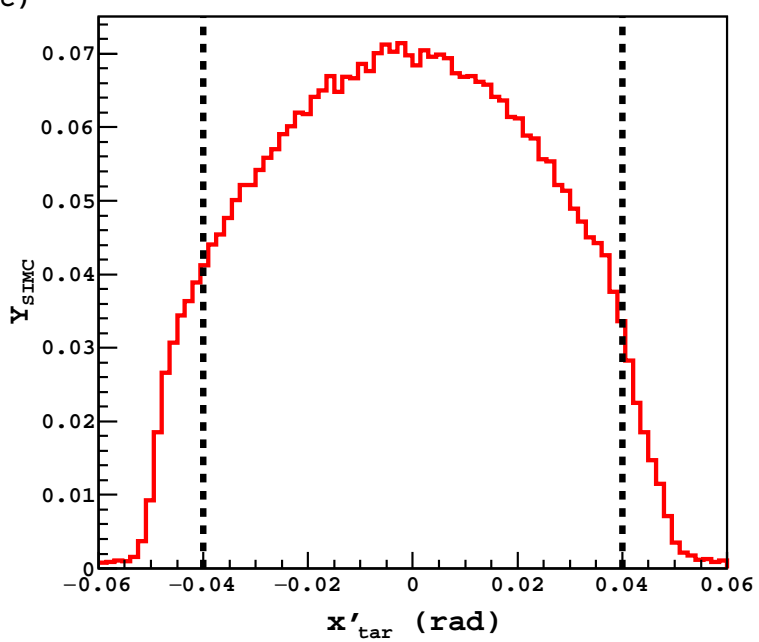

b)

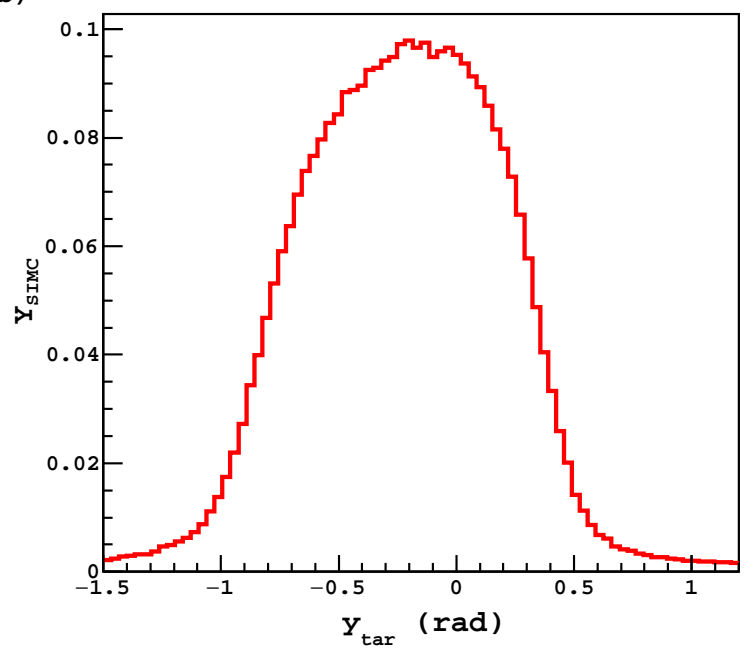

d)

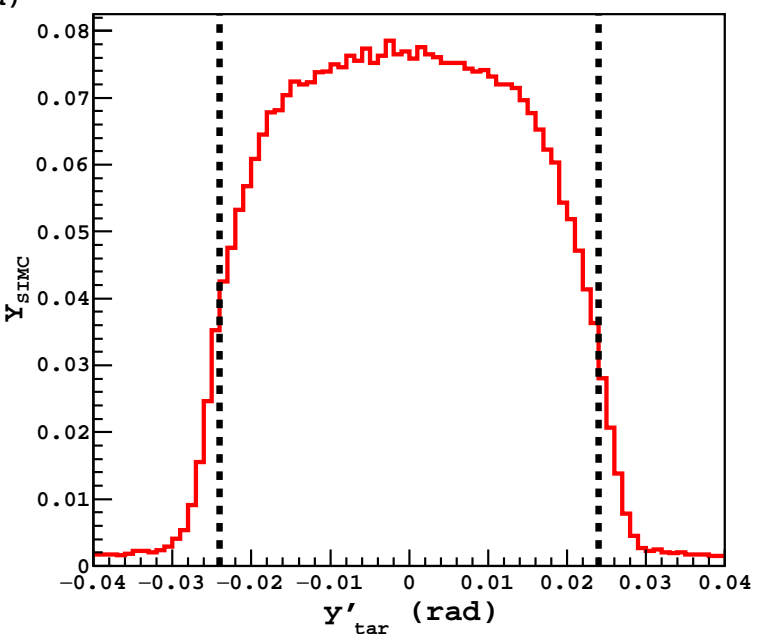

Figure 3.6: Normalized distributions for the SHMS reconstructed target quantities for $Q^{2}=$ $3.00 \mathrm{GeV}^{2}$ and $W=3.14 \mathrm{GeV}$ at low $\varepsilon$ setting and central angle for $\Lambda$ channel. $\delta$ gives the particle momentum relative to the central momentum of the spectrometer and is shown in panel a. The point of origin for an event, $y_{\text {tar }}$ is illustrated in panel b. Panels c and $\mathrm{d}$ show two angles $x_{t a r}^{\prime}$ and $y_{t a r}^{\prime}$ that characterize the particle trajectory. The cuts on each of the target quantities except $y_{t a r}$ are represented with dashed lines. Since the input to the SIMC does not take in the information about the origin of the event, there is not need to place a cut on $y_{\text {tar }}$.

order to select appropriate physics events. Such cuts on the target quantities are commonly known as spectrometer acceptance cuts. The primary purpose of the acceptance cuts is to reduce sensitivity to the edges of the spectrometer acceptance, which may be less wellunderstood than the central regions. The acceptance cuts on HMS used for this analysis are 
based on previous studies done on the performance and acceptance of the HMS with various experiments $[3,10,2]$, whereas the design parameters of the SHMS, as shown in Table 3.1, are used to determine the appropriate SHMS acceptance cuts. These cuts are shown with dashed lines in Figure 3.6. The exact values of these cuts are provided in Subsec. 3.4.4, along with a brief discussion on all the analysis cuts applied to the simulated data.

\subsection{2 $Q^{2}-W$ Coverage}

The range of acceptance of the $Q^{2}$, and $W$ is shown in Figure 3.7. As illustrated by the figure, $Q^{2}-W$ coverage at the high $\varepsilon$ setting is larger than at the low $\varepsilon$ setting. It is also worthy to note that other kinematic variables, such as $-t$ and $\theta_{K q}$, are correlated with $Q^{2}$ and $W$, and thus their values change across the $Q^{2}-W$ coverage. This makes the determination of the separated experimental cross sections complicated, since the kinematic regions over which these cross sections are averaged are not the same for the high and low $\varepsilon$ settings. This issue can be addressed by restricting the kinematic limits so that the integration is performed over the same region for both the $\varepsilon$ settings.

Since the $Q^{2}-W$ coverage for the high $\varepsilon$ setting is larger than at low $\varepsilon$ due to the larger HMS momentum acceptance at high $\varepsilon$, the latter is used to define the kinematic region for integration. Once the kinematic limits are specified, a $Q^{2}-W$ cut, commonly referred to as the diamond cut, is placed on the high $\varepsilon$ setting, making the integration limits the same for both settings (see Figure 3.7). Although placing such additional cuts on the available experimental phase space is not desirable due to significant loss of statistics; it is necessary, however, as the extraction of reliable separated cross sections takes priority in this scenario. 


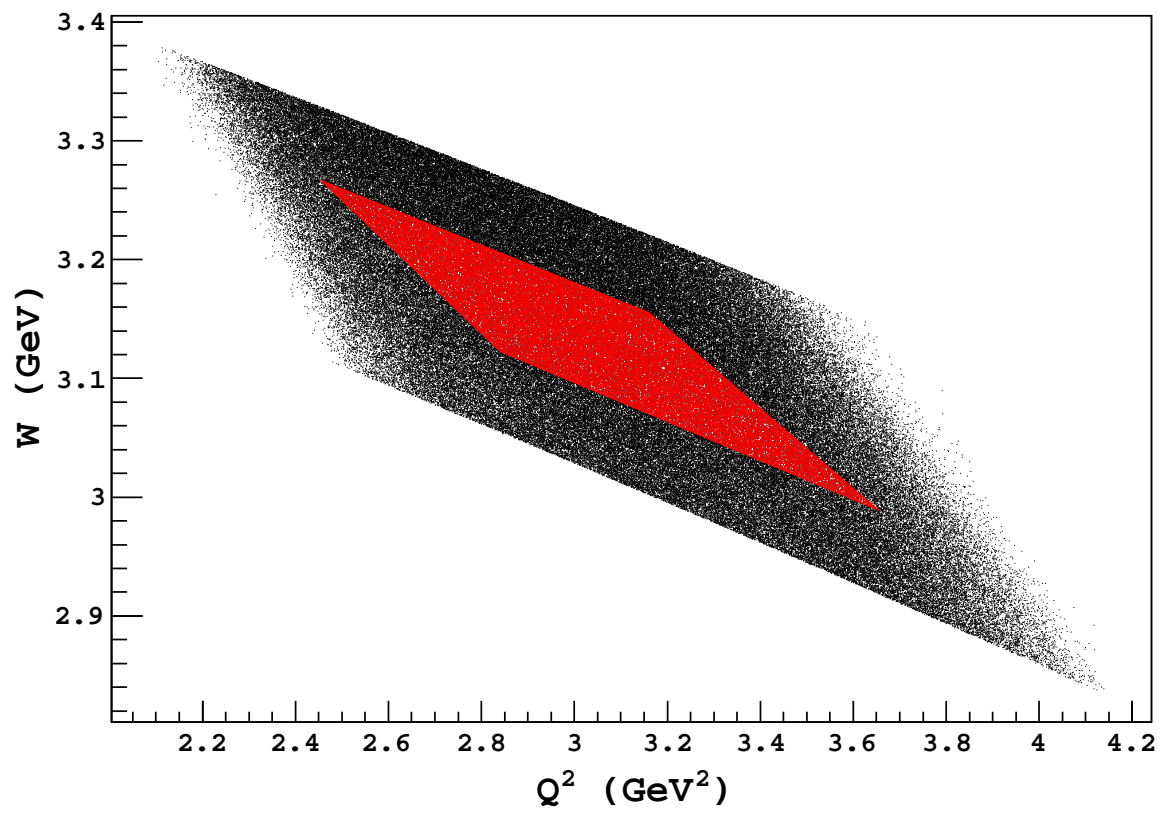

Figure 3.7: Simulated $W$ versus $Q^{2}$ coverage for $Q^{2}=3.00 \mathrm{GeV}^{2}$ and $W=3.14 \mathrm{GeV}$ setting and central angle for $\Lambda$ channel. The red data points correspond to the low $\varepsilon$ setting and are used to define the kinematic limit for the integration while the black ones represent the high $\varepsilon$ setting.

\subsubsection{Missing Mass Separation of $\Lambda$ and $\Sigma^{0}$}

The unobserved exclusive final states, $\Lambda$ and $\Sigma^{0}$, will be identified via missing mass, $M_{x}$, which is reconstructed from the scattered electron and recoiled proton four-momenta using Equation 2.6. Figure 3.8 shows histograms of simulated normalized missing masses with apparent peaks at $\Lambda$ and $\Sigma^{0}$ of $1.11568 \mathrm{GeV}$ and $1.19264 \mathrm{GeV}$, respectively [1]. With the experimental data, the tails are expected to slightly taper toward higher missing mass from both the $\Lambda$ and $\Sigma^{0}$ peaks. Such artifacts in the missing mass distribution are largely because of the effects of radiative processes. In these processes, the incident/scattered electron loses some energy by radiating a photon, resulting in some extra energy and momentum "missing" from what is being read out by the DAQ. In addition, the electroproduced $K^{+}$also loses some energy due to multiple scattering and ionization. These effects lead to an apparent increase in the reconstructed missing mass. However, based on the work of Mo and Tsai 
$[55,56]$, extended to be valid for a coincidence framework [35], the corrections to such effects are implemented in SIMC while simulating the $p\left(e, e^{\prime} K^{+}\right) \Lambda\left(\Sigma^{0}\right)$ reaction. The details of the corrections, which consider both internal and external radiation, are well-documented in several references $[33,35]$. SIMC also takes into account the collimator in the analysis via Monte Carlo technique, similar to the one detailed in Ref. [11], in order to properly characterize the acceptance of the spectrometers.

Furthermore, the possibility of kaons decaying on their way from the target station to the detector huts is also included in SIMC in a following way. Taking a kinematics setting with $K^{+}$momentum of $5.189 \mathrm{GeV} / c$ and using $K^{+}$rest mass of $0.493 \mathrm{GeV}$ [1], the relativistic

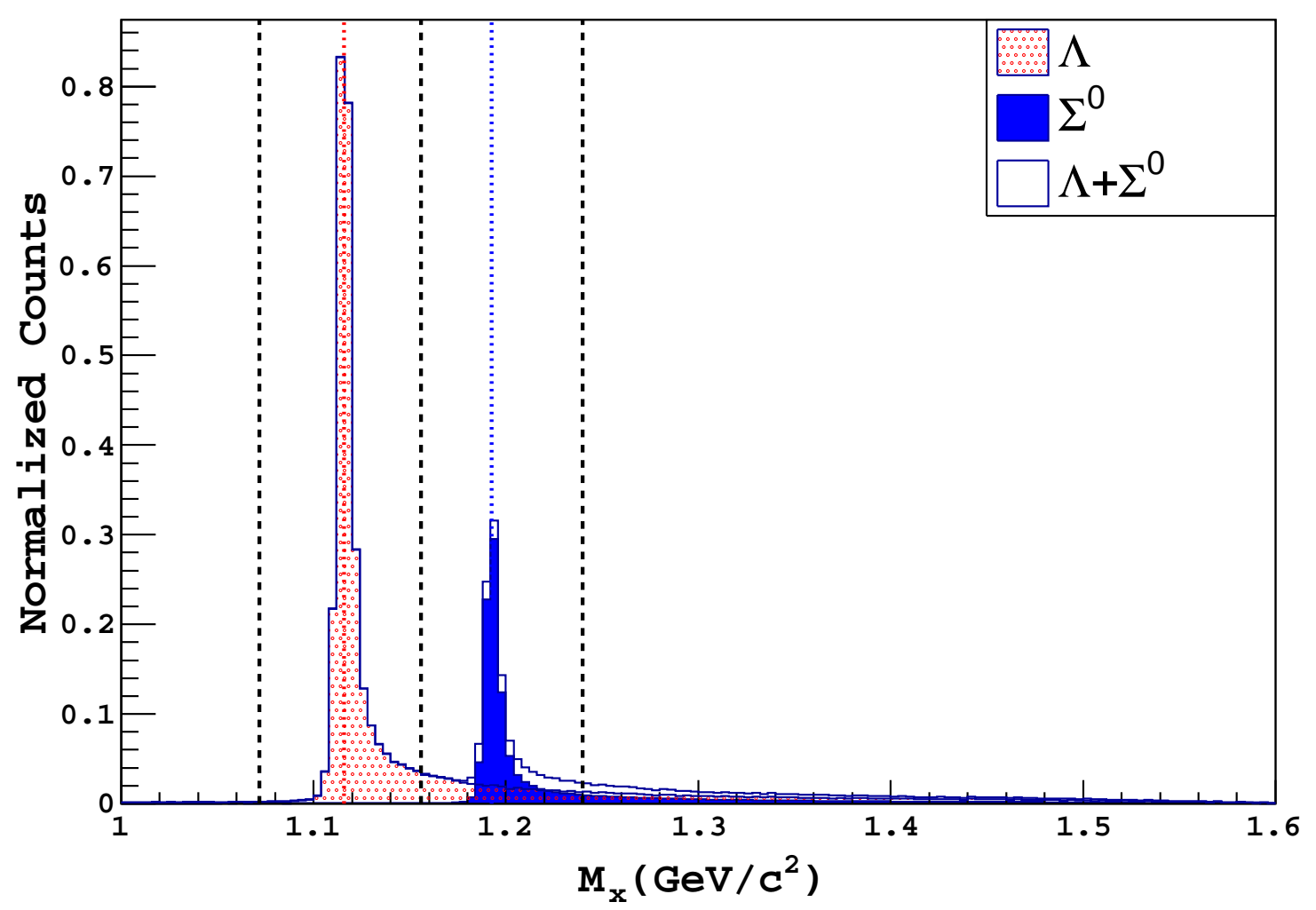

Figure 3.8: Normalized missing mass distributions for $\Lambda$ and $\Sigma^{0}$ final states for the $Q^{2}=2.0$ $\mathrm{GeV}^{2}$ and $\mathrm{W}=3.14 \mathrm{GeV}$ setting at low $\varepsilon$ and central SHMS angle using the VR and the RPR models. The sum of the $\Lambda$ and $\Sigma^{0}$ is also shown. The red and blue vertical dotted lines represent the accepted $\Lambda$ and $\Sigma^{0}$ masses, respectively [1], whereas the black dashed lines represent cuts to separate the final states. 
factors, $\beta$ and $\Gamma$, are calculated to be 0.996 and 10.56, respectively. Then, the Lorentz dilated lifetime $(\tau)$ of $K^{+}$is given by a product of $\Gamma$ and proper lifetime of $K^{+}(12.4 \mathrm{~ns}[1])$, which is calculated to be 131.1 ns. The pathlength from the target to the calorimeter of the SHMS is roughly $22 \mathrm{~m}$ and the $K^{+}$velocity in the lab-frame is $\beta c$ so the time taken by $K^{+}$ to travel the pathlength is $t$ is $73.34 \mathrm{~ns}$. Now the probability of survival (i.e. probability of $K^{+}$traversing the pathlength without decaying) is given by,

$$
P(\text { survival })=e^{\frac{-t}{\tau}}=e^{-73.34 / 131.1}=0.57
$$

For the kinematics setting selected, about $60 \%$ of the electroproduced kaons will survive when they travel through the pathlength of $22 \mathrm{~m}$ in the SHMS. For the simulation, the actual pathlength through the spectrometer and kaon momentum are used to compute the decay on an event-by-event basis.

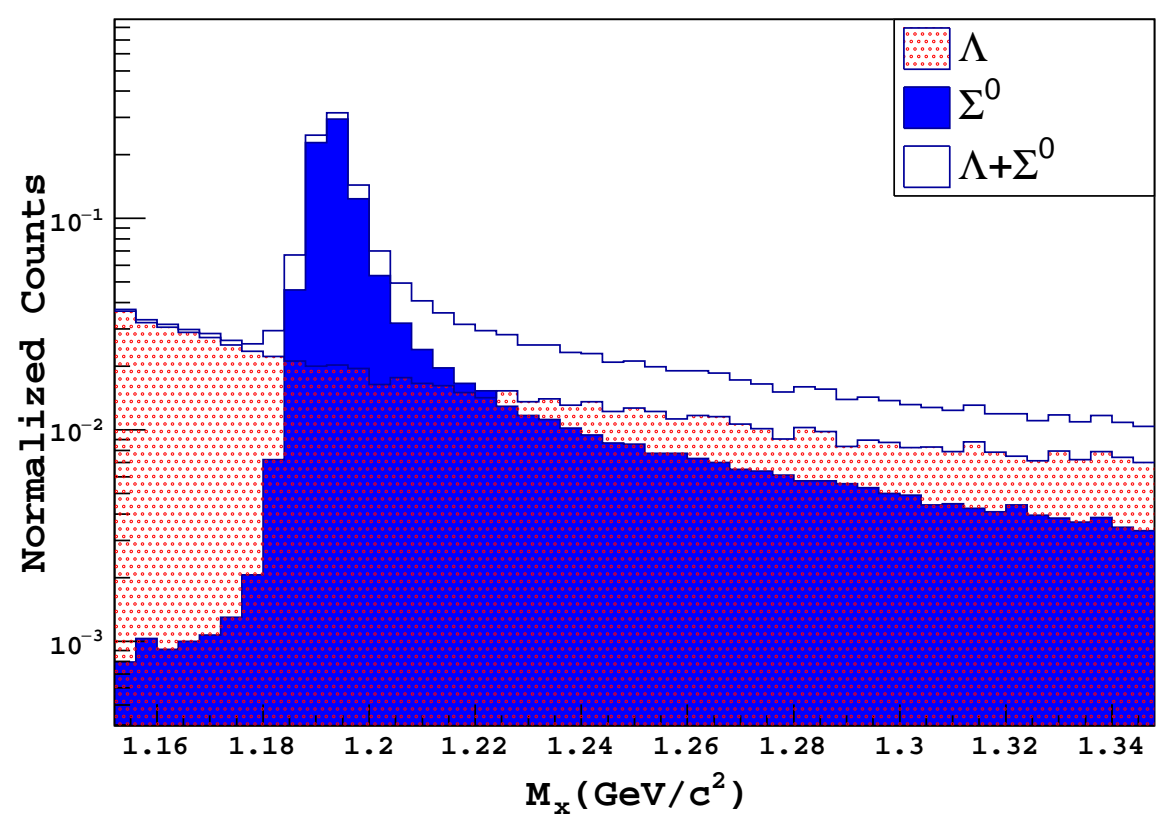

Figure 3.9: Figure 3.8 zoomed in on the $\Sigma^{0}$ missing mass distribution in log scale. A clear contamination of the $\Sigma^{0}$ missing mass distribution with the tail of the $\Lambda$ distribution can be seen in the plot. 


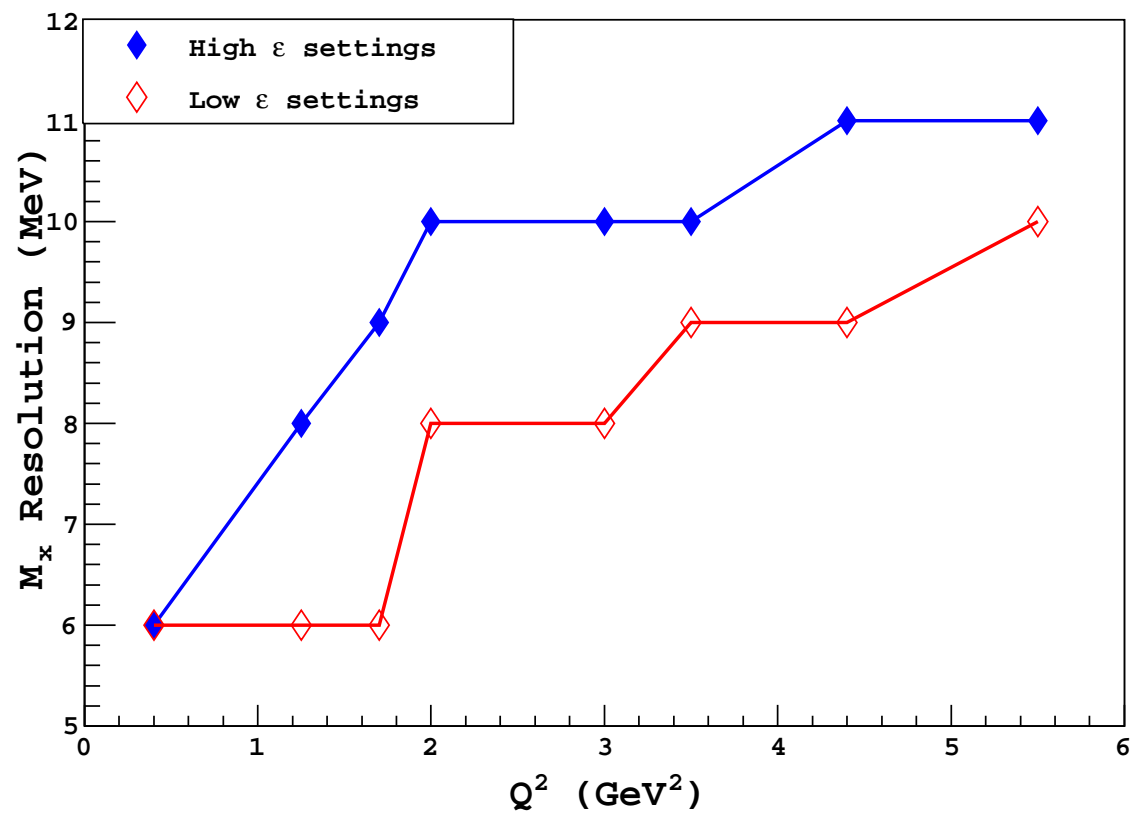

Figure 3.10: Resolution of $\Lambda$ missing mass (in $\mathrm{MeV}$ ) as a function of $Q^{2}$. The blue data points represent the low $\varepsilon$ settings while the red data points show the high $\varepsilon$ settings. $\Sigma^{0}$ final state also gives similar resolution range. The line connecting the data points only serve as a guide to the eye. The $M_{x}$ resolution as a function of $Q^{2}$ is not expected to be a smooth function due to the different spectrometer momentum and beam energy used for each setting.

A crude cut was placed on the simulated missing mass distributions to separate the $\Lambda$ and $\Sigma^{0}$ reaction channels. The value of this cut is shown in Table 3.3. However, a detailed study of the cut dependences has to be made using both simulated and experimental data before the cut value is finalized. In addition, the analysis of the final states become complicated because of the contribution from the $\Lambda$ tail to the $\Sigma^{0}$ missing mass distribution. The study of the simulated missing mass distribution across all the kinematic settings suggests that the contamination of $\Lambda$ tail is $\sim 20 \%$ for the worst case scenario. This would have to be taken into account via detailed peak shape fitting in the actual experimental analysis. However, for the purposes of run planning of this study, the crude cut on missing mass is sufficient. Similarly, the resolution of the missing mass distribution was also studied and found out to be roughly $\sim 10 \mathrm{MeV}$, which should be more than adequate to separate out the final 
states. Figure 3.10 shows missing mass resolutions plotted against $Q^{2}$ at both high and low $\varepsilon$ settings.

\begin{tabular}{lcc}
\hline Variable & Value & Reference \\
\hline \hline & Spectrometer Acceptance Cuts \\
\hline HMS $|\delta|$ & $\mid$ hsdelta $\mid<8.0$ & Subsection 3.4.1 \\
HMS $\left|x_{\text {tar }}^{\prime}\right|$ & $\mid$ hsxptar $\mid<0.080$ & $"$ \\
HMS $\left|y_{\text {tar }}^{\prime}\right|$ & $\mid$ hsyptar $\mid<0.035$ & $"$ \\
SHMS $|\delta|$ & $\mid$ ssdelta $\mid<15.0$ & $"$ \\
SHMS $\left|x_{t a r}^{\prime}\right|$ & $\mid$ ssxptar $\mid<0.040$ & $"$ \\
SHMS $\left|y_{\text {tar }}^{\prime}\right|$ & $\mid$ ssyptar $\mid<0.024$ & $"$ \\
\hline \multicolumn{3}{c}{ Kinematic Cuts } \\
\hline$\Lambda$ missing mass $(\mathrm{GeV})$ & $1.07<M_{x}<1.15$ & Subsection 3.4.3 \\
$\Sigma^{0}$ missing mass $(\mathrm{GeV})$ & $1.16<M_{x}<1.24$ & Figure 3.8 \\
$Q^{2}-W$ cut & - & Figure 3.7 \\
\hline
\end{tabular}

Table 3.3: Overview of the analysis cuts used to select the simulated data for extracting the relevant rates information.

\subsubsection{Analysis Cuts}

An overview of the cuts used for the study of real and accidental rates is shown in Table 3.3. The first panel contains cuts on the reconstructed target variables for both HMS and SHMS. The spectrometer acceptance cuts help reduce sensitivity to the edges of the acceptance and outside the limits of these cuts, the quality of the reconstruction worsens. The second panel of the table consists of cuts on the physics quantities such as $W, Q^{2}$, and the missing mass. The diamond-shaped cuts on $W$ and $Q^{2}$ are outlined to entail the kinematic ranges of low $\varepsilon$ data for both the high and low $\varepsilon$ settings. The missing mass cuts are used to separate the $\Lambda$ and $\Sigma^{0}$ final states and the ranges were chosen taking into consideration both the missing mass resolutions and the accepted masses of $\Lambda$ and $\Sigma^{0}$. The cuts were placed $\sim 40 \mathrm{MeV}$ on both sides of the missing mass peaks. For the extraction of cross sections, the dependence on the cuts has to be studied by varying the ranges of cuts and studying the change in calculated cross sections. 


\subsection{Projected Real Coincidence Rates}

For the estimation of real and accidental coincidences, we have assumed the length of the liquid hydrogen target to be $10 \mathrm{~cm}$ and the beam current for the cryo-target runs to be $70 \mu \mathrm{A}$ for all of the kinematic settings. These values are slightly different than the ones used in the proposal and are used after the consultation with the Jefferson Lab management [57]. Since the target end windows will be in the acceptance of both spectrometers in all configurations, background subtraction is necessary. "Dummy" targets, which consists of two

\begin{tabular}{ccccccccccc}
\hline$Q^{2}$ & $\varepsilon$ & $\mathrm{R}\left(\pi^{+}\right)$ & $\mathrm{R}\left(K^{+}\right)$ & $\mathrm{R}(p)$ & $\mathrm{R}\left(\pi^{-}\right)$ & $\mathrm{R}\left(K^{-}\right)$ & $\mathrm{R}\left(e^{-}\right)$ & $\Lambda$ & $\Sigma^{0}$ & $\mathrm{R}(\mathrm{acc})$ \\
& & & & & & & & $\mathrm{R}(\mathrm{real})$ & $\mathrm{R}(\mathrm{real})$ & \\
$\mathrm{GeV}^{2}$ & & $\mathrm{kHz}$ & $\mathrm{kHz}$ & $\mathrm{kHz}$ & $\mathrm{kHz}$ & $\mathrm{kHz}$ & $\mathrm{kHz}$ & $\mathrm{Hz}$ & $\mathrm{Hz}$ & $\mathrm{Hz}$ \\
\hline \hline 0.40 & 0.411 & 468.3 & 61.5 & 92.8 & 254.3 & 4.6 & 22.9 & 0.557 & 0.052 & 828.7 \\
0.40 & 0.685 & 998.7 & 124.5 & 159.4 & 222.5 & 7.0 & 205.0 & 1.262 & 0.127 & $\mathbf{1 0 9 8 8 . 3}$ \\
1.25 & 0.492 & 149.2 & 50.0 & 34.5 & 114.6 & 6.1 & 19.9 & 0.787 & 0.309 & 231.1 \\
1.25 & 0.699 & 416.6 & 116.9 & 71.5 & 70.6 & 5.4 & 111.0 & 1.300 & 0.442 & $\mathbf{2 7 5 9 . 6}$ \\
2.00 & 0.395 & 76.1 & 29.9 & 21.2 & 53.8 & 2.5 & 4.5 & 0.188 & 0.065 & 34.2 \\
2.00 & 0.580 & 234.9 & 76.8 & 45.7 & 32.5 & 2.3 & 18.6 & 0.276 & 0.085 & 285.8 \\
2.00 & 0.752 & 311.7 & 96.0 & 60.6 & 17.4 & 1.7 & 85.9 & 0.353 & 0.108 & 1623.6 \\
3.00 & 0.391 & 36.4 & 16.8 & 12.3 & 19.8 & 1.0 & 2.2 & 0.095 & 0.030 & 8.0 \\
3.00 & 0.691 & 143.3 & 52.2 & 33.6 & 6.1 & 0.6 & 23.4 & 0.146 & 0.041 & 216.8 \\
\hline 1.70 & 0.595 & 80.1 & 20.8 & 35.1 & 18.2 & 0.7 & 11.9 & 0.329 & 0.184 & 68.9 \\
1.70 & 0.856 & 163.4 & 39.4 & 64.0 & 6.6 & 0.5 & 165.0 & 0.663 & 0.289 & 1764.1 \\
3.50 & 0.364 & 28.1 & 15.1 & 9.3 & 19.0 & 1.0 & 1.8 & 0.072 & 0.018 & 5.4 \\
3.50 & 0.557 & 189.4 & 76.1 & 35.3 & 8.7 & 0.8 & 7.7 & 0.077 & 0.022 & 96.6 \\
\hline 3.00 & 0.634 & 24.9 & 7.7 & 15.3 & 2.1 & 0.08 & 4.8 & 0.115 & 0.063 & 9.4 \\
3.00 & 0.888 & 37.8 & 10.8 & 22.9 & 0.5 & 0.04 & 72.0 & 0.217 & 0.109 & 206.2 \\
4.40 & 0.479 & 12.7 & 5.7 & 6.1 & 2.2 & 0.1 & 1.3 & 0.050 & 0.020 & 1.4 \\
4.40 & 0.735 & 32.5 & 12.3 & 12.6 & 0.6 & 0.05 & 9.7 & 0.068 & 0.020 & 22.3 \\
5.50 & 0.372 & 10.3 & 5.7 & 4.4 & 2.5 & 0.1 & 0.5 & 0.020 & 0.006 & 0.5 \\
5.50 & 0.562 & 49.0 & 22.2 & 14.2 & 0.9 & 0.07 & 2.2 & 0.021 & 0.005 & 7.6 \\
\hline
\end{tabular}

Table 3.4: Estimated HMS and SHMS single rates, as well as real and accidental coincidence rates, for all the kinematic settings of the E12-09-11 experiment. The real rates listed in the table uses VR model for $\Lambda$ final state and RPR-2007 model for $\Sigma^{0}$ final state [40, 43]. The calculation also assumes $10 \mathrm{~cm}$ liquid hydrogen target and the beam current of $70 \mu \mathrm{A}$. The accidental rates assume a resolving time of $40 \mathrm{~ns}$ and correspond to the online rates. The effects of accident coincidences are expected to be effectively eliminated once the analysis cuts are applied. Note: Only the most forward angle settings are represented in the table since the reals are higher for these settings than for settings at other angles. 
thin aluminum pieces separated by a length equivalent to the cryo-target, are used to study the background contributions from the target end windows. A thick dummy target - roughly a factor of 8 thicker than the cryo-target - will be used, allowing for a rapid accumulation of counts for the background measurement. Assuming a maximum beam current of $30 \mu \mathrm{A}$ for the dummy target runs, background measurements will be faster than the cryo-target runs by a factor of 3 . The thicknesses of the cryogenic and dummy targets have to be taken into account while performing the analysis of the experimental data. However, while simulating the $p\left(e, e^{\prime} K^{+}\right) \Lambda\left(\Sigma^{0}\right)$ reaction, SIMC assumes no contribution due to the target end windows. Thus, the dummy subtraction as well as thickness correction are not required for the analysis of the simulated data.

In order to estimate the expected real coincidence rates for both $\Lambda$ and $\Sigma^{0}$ channels, the normalized simulation yields have to be calculated for both channels. The simulation yields

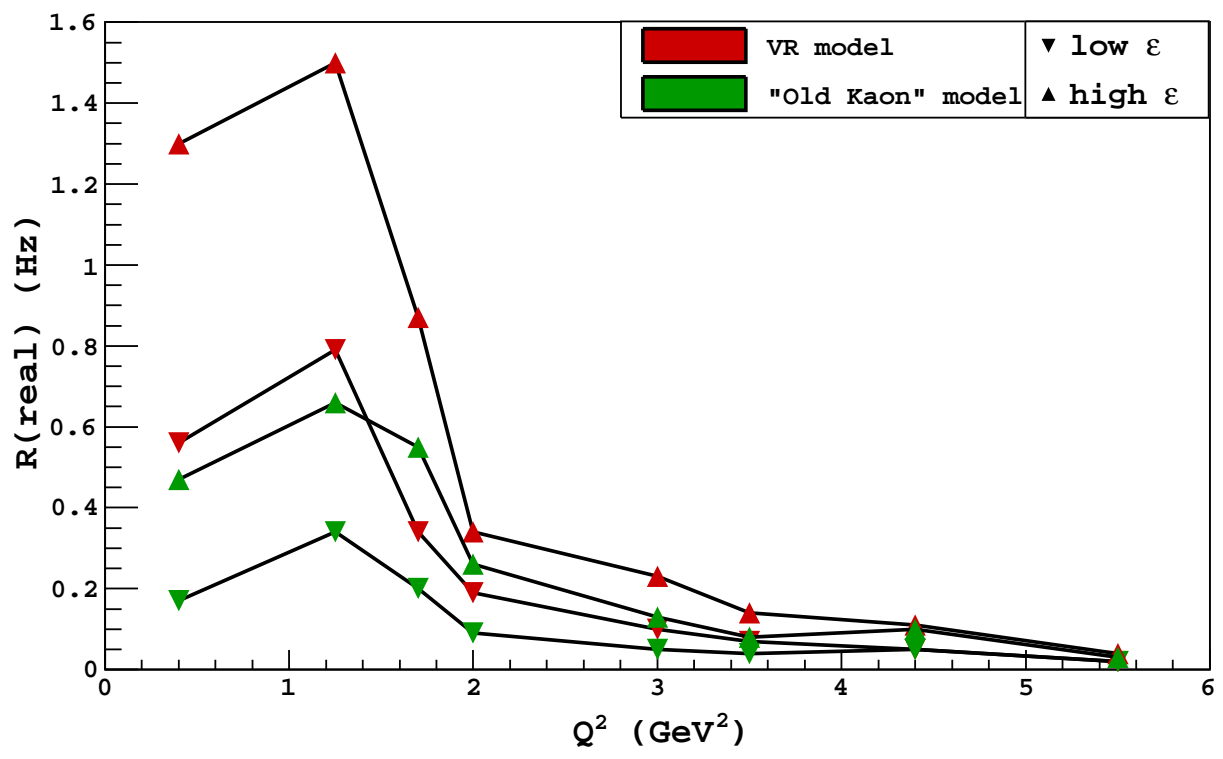

Figure 3.11: Real coincidence rates calculated using two models versus $Q^{2}$ at central angle settings for $\Lambda$ final state. The reals denoted by red data points are calculated using the VR model whereas the green data points represent the reals with the "Old Kaon" model. Similarly, the reals for high $\varepsilon$ setting are depicted with solid triangles while the reals for low $\varepsilon$ is illustrated using solid upside-down triangles. Also, the line connecting the data points only serve as a guide to the eye, and does not describe the reals as a function of $Q^{2}$. 
are given by the total numbers of events underneath the missing mass distributions (see Sec. 2.4), conventionally normalized to $1.0 \mathrm{mC}$ of total experimental beam charge. Once the simulated yield is obtained for each setting for both the final states, it is simply divided by the total time, which is calculated to be $\sim 14.286 \mathrm{~s}$ (using the total experimental charge of $1.0 \mathrm{mC}$ and the beam current of $70 \mu \mathrm{A}$ ), to calculate the real coincidence rate. The results for the expected real coincidence rates at the most forward angle settings (angles along the $\vec{q}$ for low $\varepsilon$ and the negative angle settings with respect to the $\vec{q}$ for high $\varepsilon$ ) are shown in Table 3.4 for both $\Lambda$ and $\Sigma^{0}$ final states. The real coincidence rates for the $\Lambda$ channel calculated using the VR model ranges from $0.02 \mathrm{~Hz}$ for the highest $Q^{2}$ setting of $5.50 \mathrm{GeV}^{2}$ to 1.50 $\mathrm{Hz}$ at the low $Q^{2}$ settings of 0.40 and $1.25 \mathrm{GeV}^{2}$, while the $\Sigma^{0}$ reals, calculated using the RPR-2007 model, are lower than the $\Lambda$ ones roughly by an order of magnitude. Figure 3.11 shows the comparison between two models, namely VR and "Old Kaon", for $\Lambda$ channel at high and low $\varepsilon$ settings. It can seen from the plot that the disagreement between the models is much higher at low $Q^{2}$ than at high $Q^{2}$.

\subsection{Projected Dominant Backgrounds}

Throughout the entire running of the E12-09-11 experiment, the SHMS and HMS will be set to positive and negative polarities, respectively. Thus, the $\left(e, \pi^{+}\right),\left(e, K^{+}\right)$and $(e, p)$ reactions primarily contribute towards the singles rates into the SHMS. Similarly, singles rates of the HMS are dominated primarily due to $\left(e, e^{\prime}\right),\left(e, \pi^{-}\right)$, and $\left(e, K^{-}\right)$reactions. The $\left(e, e^{+}\right)$reaction in the SHMS and the $\left(e, p^{-}\right)$reaction in the HMS does not contribute towards the singles rates, since their rates are much lower than the dominant background reactions mentioned above. The singles rates from these reactions can result in accidental coincidences which are a source of background for the measurement of the exclusive $K^{+}$ electroproduction above the resonance region. The singles rates into both spectrometers 
were estimated and summarized in Table 3.4. In projecting the singles rates for electrons, a program called "electron scattering" was used, which is a Bosted/Christy parametrization of the global elastic and inelastic electron cross sections [58], whereas the hadron singles rates were estimated using "wiser" program, a parameterization developed by Steve Rock at Standford Linear Accelerator (SLAC) based on a fit to experimental pion, kaon, and nucleon photoproduction at higher beam energy ranging from 5.0 to $19.0 \mathrm{GeV}$ [59]. The luminosity information in the wiser code was set to $\sim 1.90 \times 10^{38} \mathrm{~cm}^{-2} \cdot \mathrm{s}^{-1}$, based on the beam current, target length, and target density for the experiment given by Equation 2.8.

The projected singles rates in both spectrometers are well below the anticipated capabilities of the individual spectrometer DAQs, which can accommodate singles rates in the multi-kHz range (See Table 3.1). In the SHMS, the singles rates range from a few $\mathrm{kHz}$ to 1.0 MHz. However, only a fraction of these events will be written to the disk by using singles prescale of about 1000:1. Even though singles rates at high $Q^{2}$ are comparable for all the hadrons transversing the SHMS focal plane, $\pi^{+}$singles are dominant for the proposed kinematics with the rates greater than eight times the $K^{+}$singles and six times the $p$ singles at the lowest $Q^{2}$ settings. This should not be an issue since the detector combination of aerogel and heavy gas C̆erenkov will provide good $K^{+}$particle identification over the momentum range of the proposed measurement. Furthermore, clean $\left(e, e^{\prime} K^{+}\right)$identification will be done offline via various analysis cuts since the online data sample will likely be dominated by the $\pi^{+}$coincidences, which can be analyzed to yield additional physics information. The HGC will provide $\pi^{+} / K^{+}$separation, with rejection ratio expected to be better than $100: 1$ for $p_{S H M S}$ of $3.4 \mathrm{GeV} / \mathrm{c}$ and $10^{4}: 1$ at $p_{S H M S}$ of $8.0 \mathrm{GeV} / \mathrm{c}$. Likewise, $K^{+} / p$ separation will be provided by the aerogel ČCerenkov for the SHMS momenta between 2.6 and $7.1 \mathrm{GeV} / \mathrm{c}$, where a rejection ratio of $300: 1$ is expected [51]. For the HMS, the singles rates span from a few hundred $\mathrm{Hz}$ to a few hundreds of $\mathrm{kHz}$, which is well within the operating parameters 
of previous HMS experiments. $\pi^{-}$singles dominate the low $Q^{2}$ settings, while $e^{-}$singles contribute the most for the higher $Q^{2}$ settings. The range of about $7.0 \mathrm{kHz}$ for $K^{-}$singles is quite modest compared to a few hundred $\mathrm{kHz}$ for both $\pi^{-}$and $e$ singles.

Once the singles rates are calculated, the accidental rates can be determined by,

$$
R(\text { accidental })=\left(\sum H M S_{\text {singles }}\right) \cdot\left(\sum S H M S_{\text {singles }}\right) \cdot\left(\Delta t_{\text {coin }}\right),
$$

where $\sum H M S_{\text {singles }}$ and $\sum S H M S_{\text {singles }}$ correspond to the total singles rates of the HMS and the SHMS, respectively and the coincidence window, $\Delta t_{\text {coin }}$, used for this analysis is 40 ns. In calculating the accidental coincidence rates, the hadron trigger was taken to be equal to the raw trigger. No distinction between pions, kaons, and protons was made in the SHMS trigger; however, $\pi^{-}$and $K^{-}$will be rejected at the hardware level for this experiment. The lead-glass calorimeter coupled with the gas Čerenkov will be used to identify electrons in the HMS. On the trigger level, this translates to the logical $O R$ of the high threshold pre-shower and gas C̆erenkov along with the signals from both scintillator planes. $\pi^{-}$and $K^{-}$rejection rate of 25 : 1 can be achieved with any significant loss of efficiency. Based on previous Hall C experiments, the $\pi^{-}$contamination can be effectively eliminated after applying offline cuts on calorimeter, ČCerenkov, and coincidence time. The online coincidence resolving time of 40 ns is used in the estimation of the random coincidences. The resulting online real+random rates (i.e. DAQ rates) are well within the expected capability of the HMS-SHMS DAQ except for the two lowest $Q^{2}$ data points at high $\varepsilon$ settings, which will be discussed in more detail in the final section of this chapter. These settings, with possibly problematic random coincidence rates exceeding $2.0 \mathrm{kHz}$, are represented in bold in Table 3.4. Offline, a coincidence window similar to Figure 2.11 and random subtraction as discussed in Chap. 2 will be used to greatly reduce these background contributions to a negligible value in the final experimental analysis. A brief discussion on the findings of these rate projections, as well as remaining work to be done before the running of the experiment are detailed in the 
final chapter. The updated beam time estimates will also be presented in the discussion. 


\section{Chapter 4}

\section{Discussion and Conclusion}

This chapter is dedicated to discussions of the results from the analysis of both $p\left(e, e^{\prime} \pi^{+}\right) n$ and $p\left(e, e^{\prime} K^{+}\right) \Lambda\left(\Sigma^{0}\right)$ reactions. The first half of this chapter focuses on the exclusive pion electroproduction data at high four-momentum transfer, $-t$. The discourse with regards to the analysis of $p\left(e, e^{\prime} \pi^{+}\right) n$ high $-t$ data is two-fold: it begins with the findings from the comparison of the results from our analysis to those from prior data and subsequently, the slope of the $t$-dependence of the total unseparated cross sections, combining results from this analysis, $\mathrm{F}_{\pi}-2$ data [3], and Hall $\mathrm{B}$ data [15] at high $-t$, is discussed. In the latter half, the results from the rates projections using simulated $p\left(e, e^{\prime} K^{+}\right) \Lambda\left(\Sigma^{0}\right)$ data will be examined carefully; the implications of these estimates on the experiment will also be briefly touched upon in the same section, along with a short discussion on work required to be completed in preparation of the $p\left(e, e^{\prime} K^{+}\right) \Lambda\left(\Sigma^{0}\right)$ data taking. The chapter ends with some concluding remarks on the study of QCD transition using deep inelastic exclusive meson electroproduction reactions and some concluding comments on the upcoming Jefferson Lab Hall C 12 GeV-era experiment on exclusive kaon electroproduction above the resonance region. 


\subsection{Summary for $p\left(e, e^{\prime} \pi^{+}\right) n$ analysis at high $-t$}

The experiment measured exclusive charged pion electroproduction from hydrogen at high $-t$. The data was taken for six different $-t$ settings ranging from 0.272 to 2.127 $\mathrm{GeV}^{2}$ at nominal $Q^{2}$ and $W$ of $2.50 \mathrm{GeV}^{2}$ and $2.00 \mathrm{GeV}$, respectively. Considering small contributions from the interference terms [15, 60], unseparated cross sections were determined in the lab frame at an average azimuthal angle, $\phi_{\pi}$, of $\pi$. In the final section of Chapter 2 , the extracted unseparated cross sections were compared to $Q^{2}$ and $W$ corrected prior exclusive pion electroproduction data from Halls B, and C, as well as two theoretical models. Some of the findings from those comparisons, in particular with the prior data, are elaborated below.

The results from the analysis of our high $-t$ data, as discussed earlier, were compared to $p\left(e, e^{\prime} \pi^{+}\right) n$ data from both Halls B and C. In Figure 4.1, it can seen that the agreement of the results from all three experiments is generally reasonable. Since the $\mathrm{F}_{\pi^{-}}-2$ data cover only the small $-t$ domain, the comparison between the two cross section results were made only for the two lowest $-t$ settings, represented by the black data points in the figure. Although the lowest $-t$ data points agree with each other within uncertainties, the agreement for the higher $-t$ setting is poor. This may be attributed to the different average $Q^{2}$ and $W$ coverage between these two data points because of ad hoc scaling of $Q^{2}$ applied, based on scaling studies of prior pion electroproduction cross sections valid up to relatively low $-t$ of $0.41 \mathrm{GeV}^{2}$ [39]. The average kinematic quantities, $\overline{Q^{2}}$ and $\bar{W}$ of $2.539 \mathrm{GeV}^{2}$ and 2.181 $\mathrm{GeV}$, respectively, for the lowest $-t$ setting is much closer to the average kinematics of our data (see Table 2.6) than those at the higher $-t$ setting, for which $\left(\overline{Q^{2}}, \bar{W}\right)$ is $\left(2.703 \mathrm{GeV}^{2}\right.$, $2.127 \mathrm{GeV}$ ). The polarization of the virtual photon, $\varepsilon$, for both $-t$ settings of the $\mathrm{F}_{\pi}-2$ data is 0.56 , which is also the nominal $\varepsilon$ value of these high $-t$ data. Moreover, the extraction of the unseparated cross section for $\mathrm{F}_{\pi}-2$ data took into account the contributions from the interference terms, $\sigma_{L T}$ and $\sigma_{T T}$, which this analysis only considers while determining the 


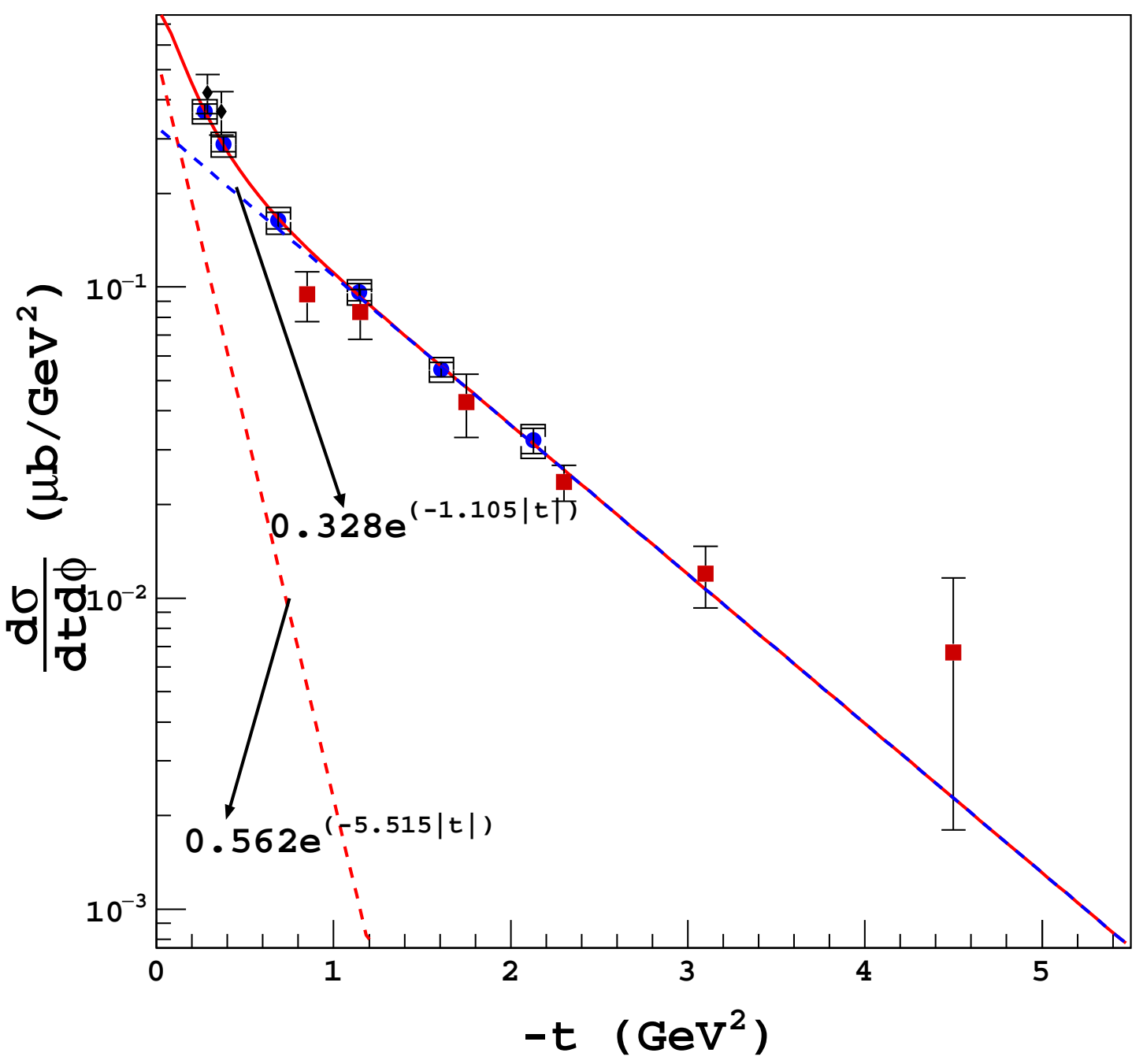

Figure 4.1: Unseparated cross sections results (combined from Halls B and C) versus $-t$. The blue data points are the results from this analysis with smaller error bars representing total random errors (statistical and random systematic added in quadrature) and the difference between the two representing the systematic uncertainties. The red square data points are the kinematically corrected results from Hall B exclusive pion electroproduction experiment and the black diamond data points at low $-t$ values are $\mathrm{F}_{\pi^{-}} 2$ unseparated cross section results. As seen in the figure, there is generally good agreement between all the results. The solid red curve, which is the sum of two dotted curves (red and blue) with equation labeled in the plot, is the parameterization of $t$-dependence of combined Halls B and C unseparated cross section results. The red dotted curve describes the soft QCD region, while the harder QCD region begins when the contributions from the soft part become negligible starting at $-t$ of $\sim 0.8 \mathrm{GeV}^{-2}$, which is described by the blue dotted curve. See text for details. (Color Online) 
model dependence of the cross sections.

Furthermore, the unseparated cross section results from CLAS at Hall B are indicated with red square boxes, in Figure 4.1. With a large acceptance, the CLAS data cover a wide range of kinematics, however the data chosen for the comparison were those closest to the average kinematics of our data. It can seen in the figure that both results agree with eachother reasonably well within the uncertainties. The cross sections determined in Hall B have a complete azimuthal, $\phi$, coverage and they were integrated over in the determination of the unseparated cross sections. Even with the limited $\phi$ coverage of our data, the ratio technique followed to determine the $t$-dependence of exclusive $\pi^{+}$electroproduction cross sections in this work was able to reproduce published cross sections results from CLAS, which follows a different method involving two separate event generators in the Monte Carlo simulation [15]. The fact that the agreement between two results is good, corroborates the technique of determining unseparated cross sections at average $\phi$ values used in this analysis.

As shown in Figure 4.1, the uncertainties in the results of this analysis are better than those of the CLAS results, which were obtained by coarsely binning their kinematic coverage in $Q^{2}$ and $x_{B}$ and determining the cross section at average values $Q^{2}$ and $x_{B}$ at each bin [15]. Further, unlike the high $-t$ data presented in this work that have clean missing mass distributions with negligible background contributions, the missing mass distributions in CLAS data contain backgrounds due to pion mis-identification and multiple pion production, contributing nearly $\sim 2.0 \%$ total systematic uncertainties [15].

The quality of the results obtained from this analysis can further be improved upon by studying the model dependence of the cross sections in a more thorough fashion. As discussed in Chapter 2, a $\phi$-dependence, based on prior data with low $-t$ range $[3,10,37]$, were used to determine the model dependence of the extracted cross sections, which dominates the systematic uncertainties. The systematics due to model dependence can be reduced 
if multiple $\phi$-dependent models are used and the extracted cross sections are compared. The comparison of the results with two theoretical models, on the other hand, was not able to discard any of the two models used. This problem can be attributed to the similar approach both models make use of while predicting the cross sections; an obvious need for L-T separated cross sections in the high $-t$ region can be inferred from the comparison.

A standard technique used in high energy physics (HEP) is the extraction of the exponential slope of the $t$-dependence of the unseparated cross section to determine the QCD regime the given DIS meson production reaction is probing [61, 62]. This is done by fitting the unseparated cross sections, $\sigma_{\text {unsep }}$, with the function of following form [61],

$$
\sigma_{\text {unsep }}=A \cdot e^{-b \cdot|t|}
$$

where $A$ and $b$ are free parameters. The parameter, $b$, in the above equation can be rigorously linked to the interaction radius for $\gamma^{*}-p$ interaction using the equation,

$$
r_{i n t}=\sqrt{|b|} \hbar c
$$

where $\hbar c=0.197 \mathrm{GeV} \cdot \mathrm{fm}$ and $r_{\text {int }}$ represents the interaction radius. In prior HEP studies $[61,62]$, this corresponds to the transverse extension of sea quarks and gluons in the proton. We make no such claim here since our data are far from the region where pQCD may be applied. Nonetheless, it provides an interesting insight into the changing character of the reaction in our kinematics.

In the same vein, the $t$ slope of the unseparated cross section (combined Halls B and C) is determined by parameterizing the $t$-dependence of cross sections. In Figure 4.1, the solid red curve, which is the sum of two dotted red $\left(-t<0.8 \mathrm{GeV}^{2}\right)$ and blue $(0.8<-t<4.5$ $\mathrm{GeV}^{2}$ ) curves, represents the parameterization obtained by performing an error-weighted fit to the combined cross section results with an equation of a form similar to that of the model cross section used in the SIMC (see Equation 2.12). The parameterization is given by a sum 
of two decaying $t$-dependent exponential functions as follows:

$$
\frac{d^{2} \sigma}{d t d \phi}=0.562 \cdot e^{-5.515 \cdot|t|}+0.328 \cdot e^{-1.105 \cdot|t|}
$$

Here, the coefficients of $|t|, 5.515$ and $1.105 \mathrm{GeV}^{-2}$, obtained by combining the cross sections results from Halls $\mathrm{B}$ and $\mathrm{C}$, are comparable to those (5.676 and $1.117 \mathrm{GeV}^{-2}$ ) obtained solely with this data. Using Equation 4.3, the interaction radii, $r_{i n t}$, are calculated for both values of the $t$-slopes and are presented in Table 4.1, along with the corresponding values determined using these data. In the low $-t$ region, the $r_{i n t}$, calculated using combined data, was found to be $0.463 \mathrm{fm}$, which is similar to the accepted $\pi^{+}$charge radius of $0.672 \mathrm{fm}[1]$. This corresponds to the forward angle exchange of meson in the $t$-channel, representing the non-perturbative soft QCD process. In contrast to $r_{\text {int }}$ of $0.463 \mathrm{fm}$ in the low $-t$ region, $r_{\text {int }}$ for the high $-t$ region is $0.207 \mathrm{fm}$, indicating that the interaction is much harder. In the high $-t$ region, the virtual photon, $\gamma^{*}$, couples directly to parton structure which is smaller than the radius of electroproduced meson, indicating that the hard QCD processes are much more important in the higher $-t$ region. This is further confirmation of the change in $t$-slopes between low and high $-t$ previously observed in electroproduction [63] and photoproduction [64].

\begin{tabular}{lc|cc|cc}
\hline \multirow{2}{*}{\begin{tabular}{c}
\multirow{2}{*}{$t$ Region } \\
\cline { 3 - 6 }
\end{tabular}} & \multicolumn{2}{|c|}{ Hange } & \multicolumn{2}{|c|}{ Hall C only } & \multicolumn{2}{c}{ Halls B + C } \\
\cline { 3 - 6 } & $\left(\mathrm{GeV}^{2}\right)$ & $\begin{array}{c}|b| \\
\left(\mathrm{GeV}^{-2}\right)\end{array}$ & $\begin{array}{c}r_{\text {int }} \\
(\mathrm{fm})\end{array}$ & $\begin{array}{c}|b| \\
\left(\mathrm{GeV}^{-2}\right)\end{array}$ & $\begin{array}{c}r_{\text {int }} \\
(\mathrm{fm})\end{array}$ \\
\hline \hline Low $-t$ & $0<-t<0.8$ & 5.676 & 0.469 & 5.515 & 0.463 \\
High $-t$ & $-t>0.8$ & 1.117 & 0.208 & 1.105 & 0.207 \\
\hline \hline
\end{tabular}

Table 4.1: The fitted results for the exponential slopes of of the $t$-dependence of cross sections and the interaction radii calculated using Equation 4.3. These quantities determined using only our high $-t$ data are listed in "Hall C only" column while "Halls B + C" column contains values determined by combining our high $-t$ data with $\mathrm{F}_{\pi^{-}}$-2 and Hall B CLAS data. There is a good agreement between the slopes and interaction radii values determined both sets of results. Note that the ranges for $-t$ regions are determined in the way explained in Figure 4.1. 


\subsection{Summary of exclusive $K^{+}$electroproduction rates}

\section{projections}

Exclusive $K^{+}$electroproduction above the resonance region will be measured for the first time by experiment E12-09-011. The unseparated cross sections will be determined following a similar technique as for the analysis of the high $-t$ data and they will be separated into polarization states of the virtual photon: L, T, LT and TT. In this analysis, the simulated $p\left(e, e^{\prime} K^{+}\right) \Lambda\left(\Sigma^{0}\right)$ data, obtained using two different models discussed in Chapter 3 , were analyzed for estimating real, and accidental coincidence rates; the results were also presented in the same chapter. The results from these rate projection studies indicate that the real coincidence rates predicted using the VR model are higher by a factor of $\sim 3$ than those obtained using the "Old Kaon" model for the lower $Q^{2}$ settings. Moreover, both the models predict significantly higher reals than those used in the proposal for all kinematic settings. This suggests that the proposed measurements of $p\left(e, e^{\prime} K^{+}\right) \Lambda\left(\Sigma^{0}\right)$ reactions might achieve notably better statistics than was originally expected. If so, this will allow a more definitive comparison of the $\Lambda$ and $\Sigma^{0}$ channels to be performed. This would have a significant positive impact upon the physics interpretation of the data, yielding crucial information on the $K^{+}$ production mechanism, and possibly improving the prospect that the $K^{+}$form factor can be extracted above the resonance region from our data for the first time. A table of the updated beam time estimates for this experiment is attached in Appendix A.

The estimated accidental coincidence rates, making up almost all of the DAQ rates, are well within the Hall C DAQ operating limits for all $Q^{2}$ settings larger than $1.25 \mathrm{GeV}^{2}$. For two the lowest $Q^{2}$ settings of 0.40 and $1.25 \mathrm{GeV}^{2}$ at high $\varepsilon$ values, the random coincidence rates surpassed 2000 events per second (i.e. $2.0 \mathrm{kHz}$ ) assuming the same beam current and target length as the other kinematic settings. In order to avoid having too low electronic livetimes 
in a commissioning experiment, when things are not yet fully understood, adjustments have to be made in order to obtain measurements at these kinematic settings. Since the random coincidence rates are proportional to the beam current as well as the target length (i.e. the luminosity), one can tackle this issue by lowering either the beam current or the target length while making these measurements. A quick scaling of the rates, lowering the beam current of 0.40 and $1.25 \mathrm{GeV}^{2}$ to 25 and $50 \mu \mathrm{A}$ respectively, reveals that, with these adjustments, the expected random coincidence rates dropped to well below the $2.0 \mathrm{kHz}$ mark.

\begin{tabular}{lccc}
\hline $\begin{array}{l}P_{S H M S} \\
(\mathrm{GeV} / c)\end{array}$ & Refractive index, $n$ & $K_{\text {npe }}^{+}$ & $p_{\text {npe }}$ \\
\hline \hline $2.6-3.0$ & 1.030 & $20-45$ & $<0.5$ \\
$3.1-3.7$ & 1.020 & $12-30$ & $<0.5$ \\
$5.2-6.3$ & 1.008 & $06-13$ & $<0.5$ \\
$6.4-7.2$ & 1.006 & $06-09$ & $<0.5$ \\
\hline \hline
\end{tabular}

Table 4.2: Aerogel Čerenkov indices of refraction for experiment, E12-09-011 for respective central SHMS momentum ranges. The number of photoelectrons (npe) expected within the spectral range of a 5" reference PMT are listed for both $K^{+}$and $p$ [51].

Further work before the start of the experiment will include the beam time estimates for ${ }^{1} \mathrm{H}\left(e, e^{\prime} p\right)$ elastic data, which are needed for the purposes of calibration and determination of kinematic offsets; the latter of the two are crucial for an L-T separation experiment. In addition, the elastic reaction also serves as an accuracy check of the modeling of the spectrometer acceptances in SIMC. This, particularly, is of importance because the SHMS is a brand new spectrometer and its magnetic optical properties (in comparison to the design specifications) are yet to be understood fully. For this experiment, the aerogel trays in the aerogel C̆erenkov detectors have to be changed depending upon the SHMS momentum and the refractive index of the aerogel $(n)$ being used, as listed in Table 4.2. A detailed time estimate for this is also necessary.

In summary, deep inelastic scattering is an effective tool to study various QCD processes, 
as indicated by its unprecedented success in shedding lights on nucleon sub-structure as well as on the properties of quarks, the constituents of QCD [6]. In this dissertation, two deep exclusive pseudoscalar meson electroproduction reactions were studied, and results were presented, along with some discussion. The soft to hard transition was studied using exclusive $\pi^{+}$electroproduction from low to high $-t$. Although the transition from soft QCD is quite evident, as provided by the $t$-slope formalism, whether the reaction is yet sufficiently hard to allow pQCD to be applied can only be determined upon further study. With the $12 \mathrm{GeV}$ upgrade of Jefferson Lab, many new opportunities involving the exploration of hadronic and partonic regimes of QCD will be available; exclusive $\pi^{+}$and $K^{+}$electroproduction being two of them. Low $-t$ data of the $p\left(e, e^{\prime} K^{+}\right) \Lambda\left(\Sigma^{0}\right)$ reactions will be used to study the $K^{+}$reaction mechanism, while data at fixed $x_{B}$ will be used to study the QCD soft-hard transition using $1 / Q^{n}$ scaling in strange systems. This is an important test to know whether one can extract Generalized Parton Distribution (GPD) information from meson electroproduction reactions in this $Q^{2}$ region. 


\section{Bibliography}

[1] K. A. Olive et al, "Review of Particle Physics," Particle Data Group, Chin. Phys., vol. C38, 2014.

[2] R. M. Mohring, A comparion of longitudinal and transverse cross sections in the $p\left(e, e^{\prime} K^{ \pm}\right) \Lambda$ and $p\left(e, e^{\prime} K^{ \pm}\right) \Sigma$ reactions. Ph.D. Thesis, University of Maryland, 1999.

[3] T. Horn, The pion charge form factor through pion electroproduction. Ph.D. Thesis, University of Maryland, 2006.

[4] R. Hofstadter R. W. McAllister, "Electron Scattering from the Proton," Phys. Rev., vol. 98, no. 217, 1955.

[5] R. Hofstadter, "Electron Scattering and Nuclear Structure," Rev. Mod. Phys., vol. 28, no. 214, 1956.

[6] I. S. Hughes, Elementary Particles. Cambridge, UK: Cambridge University Press, 1991.

[7] E. M. Henley and A. Garcia, Subatomic Physics. Hackensack, NJ, USA: World Scientific Publishing Co. Pte. Ltd., 2010.

[8] D. Griffiths, Introduction to Elementary Particles. Weinheim, Germany: Wiley-Vch Verlag GmbH \& Co., 2016.

[9] G. I. Smirnov, "Photon- and Electron-Nucleon interactions in the $1^{\text {st }}, 2^{\text {nd }}$ and $3^{\text {rd }}$ resonance regions," LPC Report PCCF RI, no. 003, 2000.

[10] J. Volmer, The pion charge form factor via pion electroproduction on the proton. Ph.D. Thesis, Virje Universiteit et Amsterdam, 2000.

[11] H. P. Blok, T. Horn, G. M. Huber, et al, "Charged pion form factor between $\mathrm{Q}^{2}=0.60$ and $2.45 \mathrm{GeV}^{2}$. I. Measurements of the cross section for the ${ }^{2} H\left(e, e^{\prime} \pi^{+}\right) n$ reaction," Phys. Rev. C, vol. 78, no. 045202, 2008.

[12] G. M. Huber, H. P. Blok, T. Horn, et al, "Charged pion form factor between $Q^{2}=0.60$ and $2.45 \mathrm{GeV}^{2}$. II. Determination of, and results for, the pion form factor," Phys. Rev. $C$, vol. 78, no. 045203, 2008.

[13] D. Gaskell, Longitudinal electroproduction of charged pions from Hydrogen, Deuterium, and Helium-3. Ph.D. Thesis, Oregon State University, 2001. 
[14] M. N. Rosenbluth, "High energy elastic scattering of electrons on protons," Phys. Rev., vol. $\mathbf{7 9}$, no. 4,1950 .

[15] K. Park, M. Guidal, et al, "Deep exclusive $\pi^{+}$electroproduction off the proton at CLAS," Eur. Phys. J. A, vol. 49, no. 16, 2013.

[16] pre-Conceptual Design Report (pCDR), SURA, 2004. Jefferson Lab Upgrade.

[17] N. Fomin, Inclusive electron scattering from nuclei in the quasielastic region at large momentum transfer. Ph.D. Thesis, University of Virginia, 2008.

[18] CEBAF Conceptual Design Report, SURA, April 1990. Basic Experimental Equipment.

[19] C. W. Leeman, D. R. Douglas, G. A. Krafft, "The Continuous Electron Beam Accelerator Facility: CEBAF at the Jefferson Laboratory," Annu. Rev. Nucl. Part. Sci., vol. 51, no. 413, 2001.

[20] G. Niculescu, First measurement of the longitudinal and transverse cross sections in ${ }^{1} \mathrm{He}\left(e, e^{\prime} K^{+}\right) \Lambda$. Ph.D. Thesis, Hampton University, 1998.

[21] K. B. Unser, "The parametric current transformer, a beam current monitor developed for LEP," AIP Conference Proceedings, vol. 252, no. 1, pp. 266-275, 1992.

[22] Beam energy measurement using the arc beam line as a spectrometer, CEBAF-PR-93004, 1993.

[23] D. G. Meekins, Coherent $\pi^{0}$ photoproduction on the deuteron. Ph.D. Thesis, College of William and Mary, 1998.

[24] B. Terburg, High energy two-body deuteron photodisintergation. Ph.D. Thesis, University of Illinois at Urbana-Champaign, 1999.

[25] J. Dunne, "Cryo and Dummy Target Information." TJNAF Hall C document, 1998.

[26] J. R. Arrington, Inclusive electron scattering from nuclei at $x>1$ and high $Q^{2}$. Ph.D. Thesis, California Institute of Technology, 1998.

[27] O. K. Baker et al, "The High Momentum Spectrometer drift chambers in Hall C at CEBAF," Nucl. Instrum. Methods Phys. Res. A, vol. 367, no. 92, 1995.

[28] W. Li, "Heavy Gas Cherenkov Detector Construction for Hall C at Thomas Jefferson National Accelerator Facility," Master's thesis, University of Regina, 2012.

[29] R. Asturyan et al, "The aerogel threshold Cherenkov detector for the High Momentum Spectrometer in Hall C at Jefferson Lab," Nucl. Instrum. Methods Phys. Res. A, vol. 548, 2005.

[30] D. van Westrum, Quasielastic (e, e'p) reactions and proton propagation through nuclei. Ph.D. Thesis, University of Colorado, Boulder, 1998. 
[31] in Proceedings of the 1995 IEEE Conference on Real-Time Computer Applications in Nuclear, Particle and Plasma Physics, 1995.

[32] V. Tvaskis, Longitudinal-Transverse separation of deep-inelastic scattering at low $Q^{2}$ on nucleons and nuclei. Ph.D. Thesis, Virje Universiteit et Amsterdam, 2004.

[33] N. C. R. Makins, Measurement of the nuclear dependence and momentum transfer dependence of quasielastic (e,e'p) scattering at large momentum transfer. Ph.D. Thesis, Massachusetts Institute of Technology, 1994.

[34] in Proceedings of the 1991 Particle Accelerator Conference, 1991. COSY INFINITY.

[35] R. Ent, B. W. Filippone, N. C. R. Makins, et al, "Radiative corrections for $\left(e, e^{\prime} p\right)$ reactions at GeV energies," Phys. Rev. C, vol. 64, no. 054610, 2001.

[36] C. J. Bebek, C. N. Brown, S. D. Holmes, et al, "Electroproduction of single pions at low $\varepsilon$ and a measurement of the pion form factor up to $Q^{2}=10 \mathrm{GeV}^{2}$," Phys. Rev. D, vol. 17, no. 1693, 1978.

[37] P. Brauel, T. Canzler, D. Cords, et al, "Separation of $\sigma_{L}$ and $\sigma_{U}$ in $\pi^{+}$-electroproduction above the resonance region," Phys. Rev. Lett., vol. B69, no. 253, 1977.

[38] B. Clasie, Measurement of nuclear transparency from $A\left(e, e^{\prime} \pi^{+}\right)$reactions. Ph.D. Thesis, Massachusetts Institute of Technology, 2006.

[39] T. Horn, "Scaling study of the pion electroproduction cross sections," Phys. Rev. C, vol. 78, no. 058201, 2008.

[40] T. Vrancx, J. Reyckebusch, J. Nys, " $K^{+} \Lambda$ electroproduction above the resonance region," Phys. Rev. C, vol. 89, no. 065202, 2014.

[41] T. K. Choi, K. J. Kong, B. G. Yu, "Pion and proton form factors in the Regge description of electroproduction $p\left(e, e^{\prime} \pi^{+}\right) n, "$ arXiv:1508.00969v1, 2015.

[42] M. E. Christy et al, "Measurement of electron-proton elastic cross-sections for $0.4<$ $Q^{2}<5.5(\mathrm{GeV} / c)^{2}, "$ Phys. Rev. C, vol. 70, no. 015206, 2004.

[43] T. Corthals, T. V. Cauteren, J. Ryckebusch, D. Ireland, "Regge-plus-resonanace treatment of the $p\left(\gamma, K^{+}\right) \Sigma^{0}$ and $p\left(\gamma, K^{0}\right) \Sigma^{+}$reactions at forward kaon angles," Phys. Rev. $C$, vol. 75, no. 045204, 2007.

[44] M. M. Kaskulov, K. Gallmeister, U. Mosel, "Deeply inelastic pions in the exclusive reaction $p\left(e, e^{\prime} \pi^{+}\right) n$ above the resonance region," Phys. Rev. D, vol. 78, no. 114022, 2008.

[45] P. Brauel et al Z. Phys. C, vol. 3, no. 101, 1979.

[46] C. N. Brown et al Phys. Rev. Lett., vol. 28, no. 1086, 1972.

[47] C. J. Bebek et al Phys. Rev. D, vol. 15, no. 594, 1977. 
[48] R. Bradford et al Phys. Rev. C, vol. 73, no. 035202, 2006.

[49] R. M. Mohring et al Phys. Rev. C, vol. 67, no. 055205, 2003.

[50] P. Markowitz, K. A. Aniol, K. Baker, et al, "Cross sectons and Rosenbluth separations in ${ }^{1} H\left(e, e^{\prime}\right) \Lambda$ up to $Q^{2}=2.35 \mathrm{GeV}^{2}, "$ Phys. Rev. C, vol. 81, no. 052201(R), 2010.

[51] T. Horn, G. M. Huber, P. M. et al, "E12-09-011, Studies of the L-T separated kaon electroproduction cross sections from 5-11 GeV." approved at JLab PAC34, 2009.

[52] S. R. Amendolia et al Phys. Rev. B, vol. 178, pp. 435-440, 1986.

[53] T. Horn Old Kaon model, 2007.

[54] M. Guidal, J. Laget, M. Vanderhaegan, "Electroproduction of strangeness above the resonance region," Phys. Rev. C, vol. 61, no. 025204, 2000.

[55] L. M. Mo Y. S. Tsai, "Radiative corrections to elastic and inelastic ep and $\mu$ p scattering," Mod. Phys., vol. 41, no. 205, 1969.

[56] Y. S. Tsai, "Radiative corrections to electron-proton scattering," Phys. Rev. Lett., vol. 122, no. 1898, 1961.

[57] R. Ent private communication, 2016.

[58] M. E. Christy, "elastic.f.” private communication, 2016.

[59] S. Rock, "wiser.f." SLAC, private communication, 2016.

[60] K. Park private communication, kpark@jlab.org, 2016.

[61] F. D. Aaron, A. Aktas, et al, "Measurement of deeply virtual Compton scattering and its $-t$ dependence at HERA," Phys. Lett., vol. B 569, no. 796-806, 2008.

[62] H. Abramowicz, L. Frankfurt, M. Strikman, "Interplay of Hard and Soft Physics in small $x$ Deep Inelastic Processes," ECONFC940808:033, no. DESY 95-047, 1995.

[63] A. Airapetian, N. Akopov, Z. Akopov, "Cross sections for hard exclusive electroproduction of $\pi^{+}$mesons on a hydrogen target," Phys. Lett. B, vol. 659, no. 486-492, 2008.

[64] M. Guidal, Photoproduction of mesons from the nucleon at intermediate energies. Ph.D. Thesis, Universite De Paris-Sud, 1996. 


\section{Appendix A}

\section{Updated beam time schedule}

The updated beam time schedule for the planned Kaon experiment is given in the table below. Note that these times are for 10,000 "good" events, however, in the proposal, we are only assuming 1000 "good" events. Thus, the time estimates presented here will be much lower if the numbers from proposal are used. 


\begin{tabular}{|c|c|c|c|c|c|c|}
\hline $\begin{array}{l}Q^{2} \\
\left(\mathrm{GeV}^{2}\right)\end{array}$ & $x_{B}$ & $\varepsilon$ & $\begin{array}{l}\mathrm{LH}_{2} \\
\text { (hrs.) }\end{array}$ & $\begin{array}{c}\text { Dummy } \\
\text { (hrs.) }\end{array}$ & $\begin{array}{c}\text { Overhead } \\
\text { (hrs.) }\end{array}$ & Total \\
\hline 0.40 & 0.072 & 0.411 & 10.3 & 1.0 & 4.0 & 15.3 \\
\hline 0.40 & 0.072 & 0.685 & 13.6 & 1.4 & 4.0 & 19.0 \\
\hline 1.25 & 0.122 & 0.492 & 9.4 & 0.9 & 4.0 & 14.3 \\
\hline 1.25 & 0.122 & 0.699 & 8.0 & 0.8 & 4.0 & 12.8 \\
\hline 2.00 & 0.182 & 0.395 & 38.3 & 3.8 & 4.0 & 46.1 \\
\hline 2.00 & 0.182 & 0.580 & 22.6 & 2.3 & 4.0 & 28.9 \\
\hline 2.00 & 0.182 & 0.752 & 21.6 & 2.2 & 4.0 & 27.8 \\
\hline 3.00 & 0.250 & 0.391 & 73.6 & 7.4 & 4.0 & 85.0 \\
\hline 3.00 & 0.250 & 0.691 & 49.4 & 4.9 & 4.0 & 58.3 \\
\hline Subtotal & reaction 1 & mechanism & 246.8 & 24.7 & 36.0 & 307.5 (12.8 days) \\
\hline 1.70 & 0.249 & 0.595 & 19.6 & 2.0 & 4.0 & 25.6 \\
\hline 1.70 & 0.249 & 0.856 & 12.2 & 1.2 & 4.0 & 17.4 \\
\hline 3.50 & 0.250 & 0.364 & 100.8 & 10.1 & 4.0 & 114.9 \\
\hline 3.50 & 0.250 & 0.557 & 90.2 & 9.0 & 4.0 & 103.2 \\
\hline Subtota & $\mathrm{x}_{B}=0.2$ & & 222.8 & 22.3 & 16.0 & 261.1 (10.9 days) \\
\hline 3.00 & 0.400 & 0.634 & 50.0 & 5.0 & 4.0 & 59.0 \\
\hline 3.00 & 0.400 & 0.888 & 32.0 & 3.2 & 4.0 & 39.2 \\
\hline 4.40 & 0.399 & 0.479 & 115.8 & 11.6 & 4.0 & 131.4 \\
\hline 4.40 & 0.399 & 0.735 & 93.6 & 9.4 & 4.0 & 107.0 \\
\hline 5.50 & 0.400 & 0.372 & 297.1 & 29.7 & 4.0 & 330.8 \\
\hline 5.50 & 0.400 & 0.562 & 295.3 & 29.5 & 4.0 & 328.8 \\
\hline \multicolumn{3}{|c|}{ Subtotal $\mathrm{x}_{B}=0.40$} & 883.8 & 88.4 & 24.0 & $996.2(41.5)$ \\
\hline \multicolumn{3}{|c|}{ Subtotals } & & & & 1564.8 \\
\hline \multicolumn{3}{|c|}{ Calibrations } & & & & 48.0 \\
\hline \multicolumn{3}{|c|}{ Beam energy switch } & & & & 48.0 \\
\hline \multicolumn{3}{|c|}{ Total } & & & & $\begin{array}{c}1660.8 \\
\text { (69.2 days) }\end{array}$ \\
\hline
\end{tabular}

Table A.1: Updated beam time estimates for E12-09-011 experiment, assuming $70 \mu \mathrm{A}$ beam current on a $10 \mathrm{~cm} \mathrm{LH}$ target. The projected number of hours includes all three (or two) $\theta_{K}$ settings. 